\title{
Advanced Development of the Spectrum Sciences Model 5005-TF, Single-Event Test Fixture
}

\author{
M. R. Ackermann \\ Preferred Parts and Emerging Technology Division \\ J. S. Browning \\ Verification Systems and Technology Division I \\ Sandia National Laboratories \\ Albuquerque, NM 87185 \\ B. W. Hughlock \\ Boeing Aerospace and Electronics, 7J-56 \\ PO Box 3999 \\ Seattle, WA 98124-2499 \\ G. K. Lum \\ Lockheed Missiles and Space Company \\ Box 3504, 0/81-63 B157-3E \\ Sunnyvale, CA 94089-3504 \\ W. C. Tsacoyeanes \\ Charles Stark Draper Laboratory \\ 555 Technology Square, $\$ 29$ \\ Cambridge, MA 0213!) \\ M. D. Weeks \\ Spectrum Sciences, Inc. \\ 3050 Oakmead Village Drive \\ Santa Clara, CA 95051
}

\begin{abstract}
A commercial application of particle identification methods commonly used in high-energy physics research has been developed for single-event testing of microelectronics. The Model 5005-'TF Single-Event Test Fixture can be used to substantially reduce the parti, qualification testing costs for many space satellite sysems.
\end{abstract}




\section{Contents}

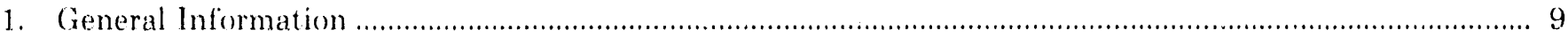

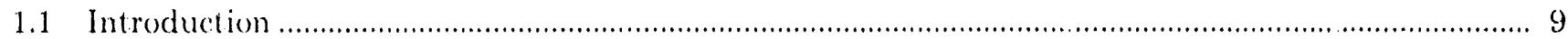

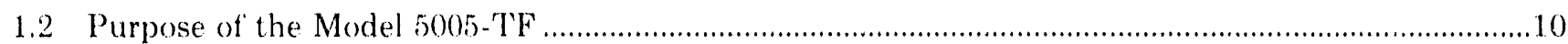

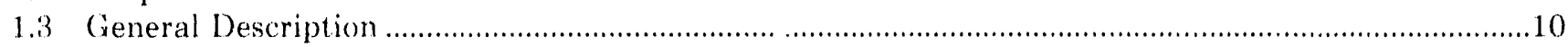

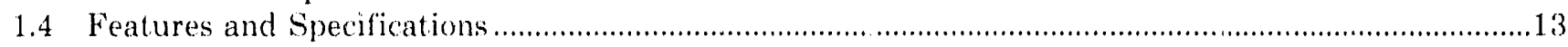

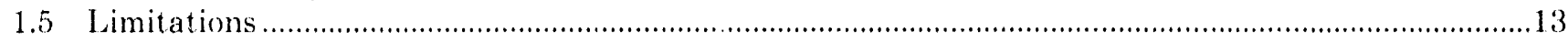

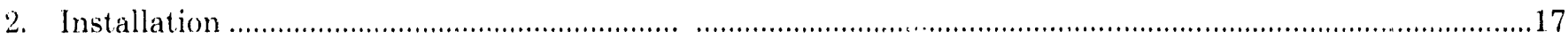

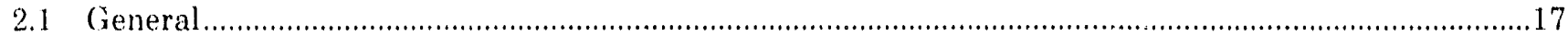

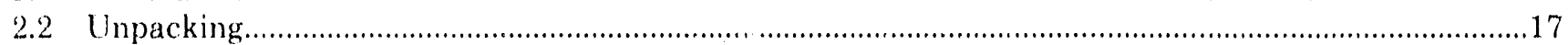

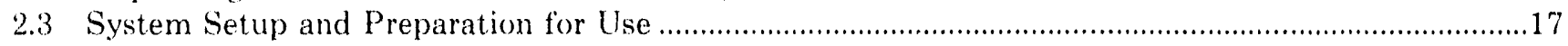

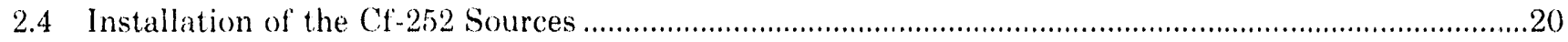

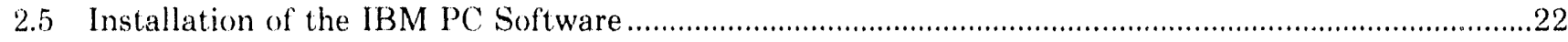

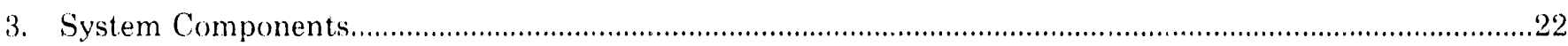

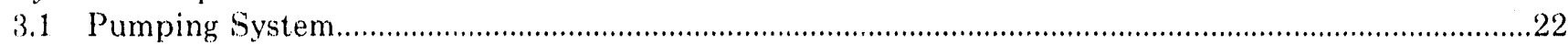

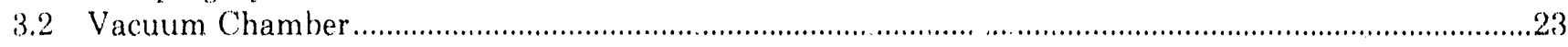

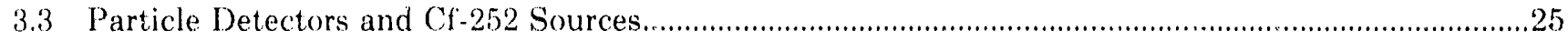

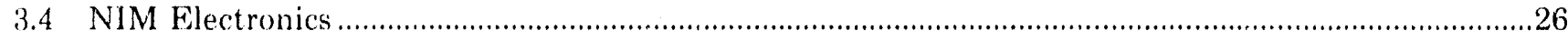

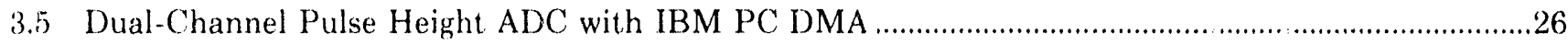

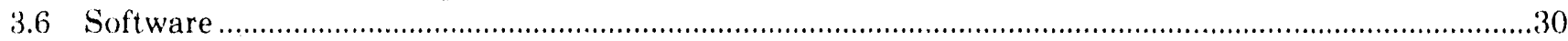

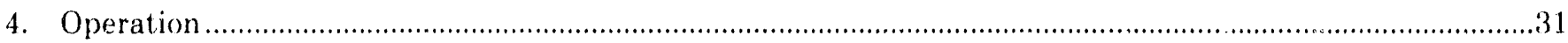

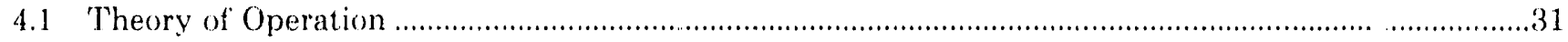

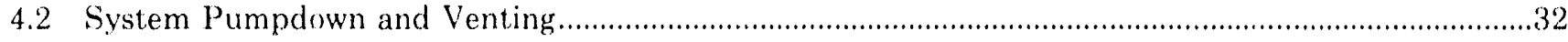

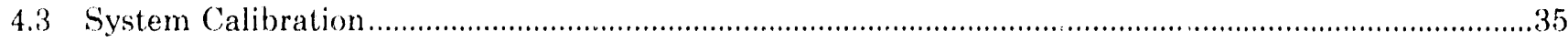

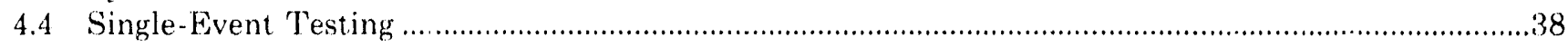

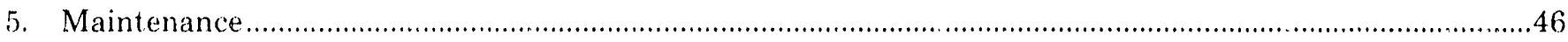

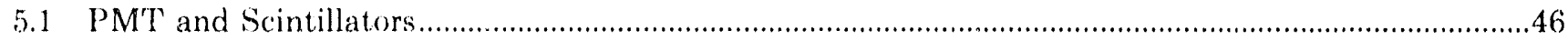

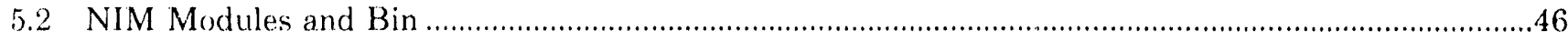

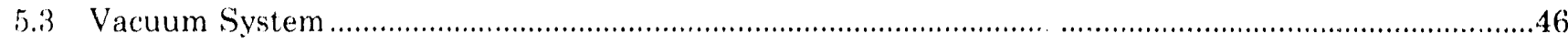

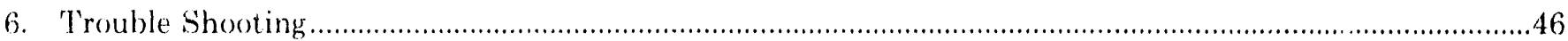

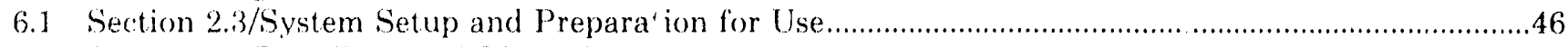

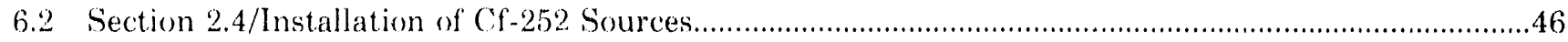

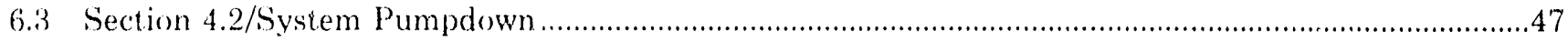

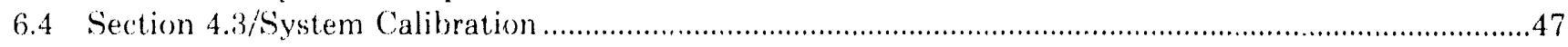

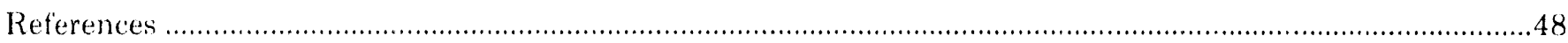

APPENDIX A Example Standard Operating Procedure for Cf -252 Sources ..........................................49

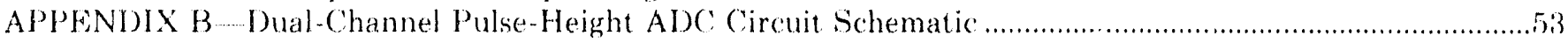

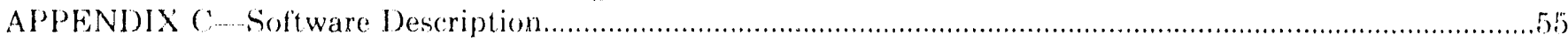

\section{Figures}

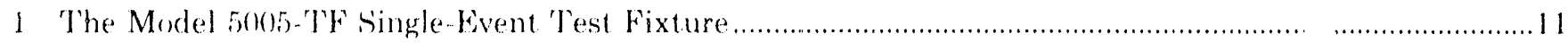

2 'TRIM computer code estimates of the average-effective LET (10", cumulative fraction in silicon) for a J)l't buried layer 


\section{Figures, continued}

3 Comparison of heavy-ion to proton-induced single event upsets ...............................................................15

4 CREME computer code estimates of the upset rates for a $10\left(1-\mu \mathrm{m}^{2} / \mathrm{bit}\right.$ cross section and a threshold LET equal to the average-effective LET given in Figure 2 ....................................................16

5 Alcatel Model 5400 turbo pump with the guard screen/O-ring seal and half-clamps ................................17

6 Flexible hose (40 in. long) used to connect the turbo pump to the roughing pump.....................................18

7 Cables used to connect the turbo pump to the rack-mounted controller.....................................................18

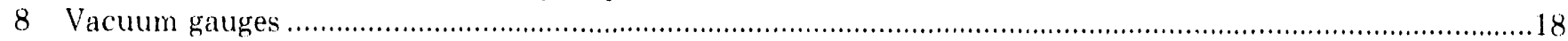

9 Ultra Torr fittings on the back side of the vacuum chamber ..............................................................18

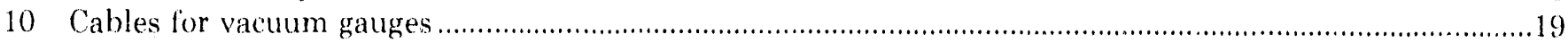

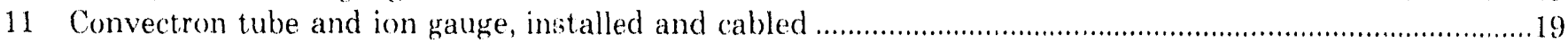

12 Clamp holder and slide rack used to position the photomultiplier tubes ...................................................19

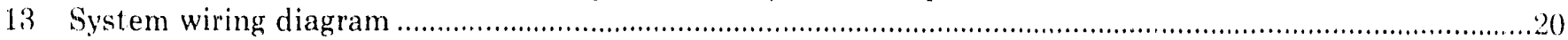

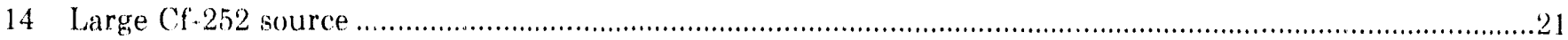

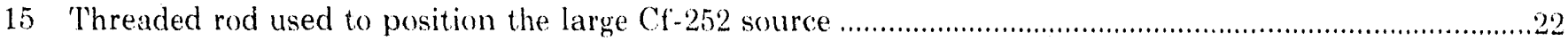

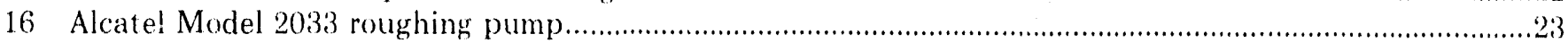

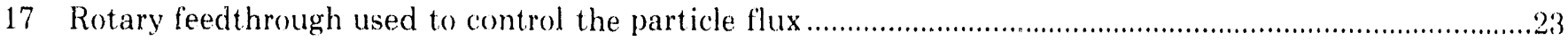

18 Borated polyethylene neutron shield used to storf the large $\mathrm{Cf}-252$ source when it is not in use..............22

19 Angle and rotation feedthrough for positioning the device under test ....................................................24

20 Fixture for mounting the device under test inside the vacuum chamber...............................................24

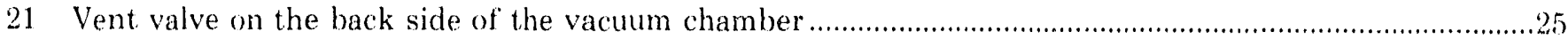

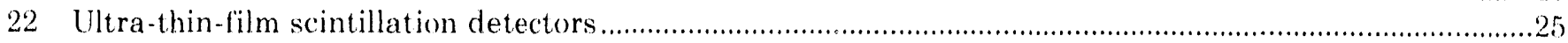

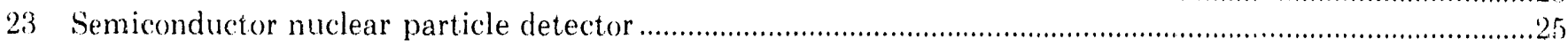

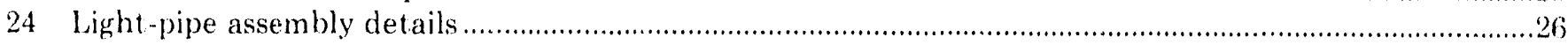

25 Setscrew used to mount the light-pipe assembly inside the aluminum holder ..........................................26

$26 \mathrm{AC}$ coupling used on the photomultiplier tube A2 output ...................................................................26

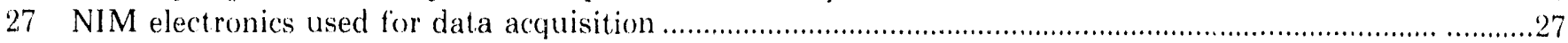

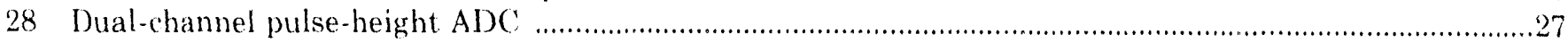

29 Functional block diagram of the dual-channel pulse-height ADC ….....................................................28

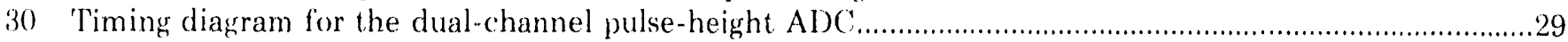

31 Menu reference for the Model 5005-TF software

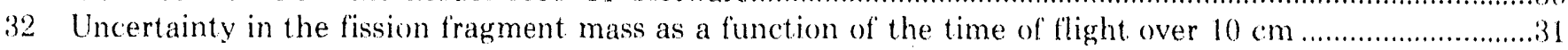

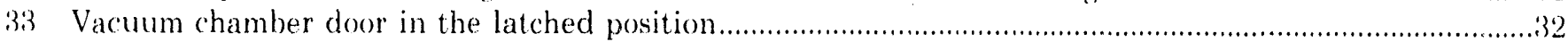

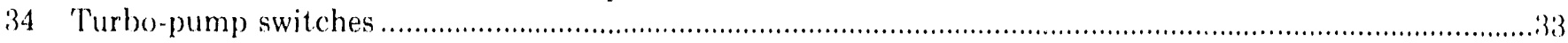

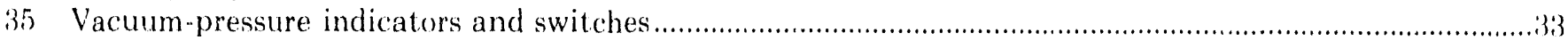

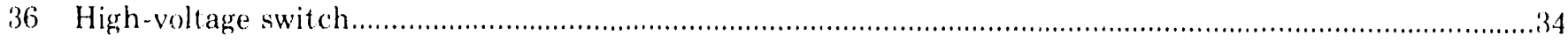

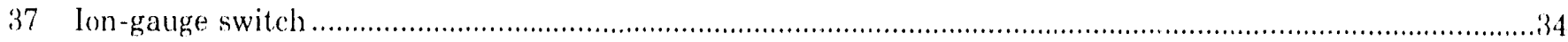

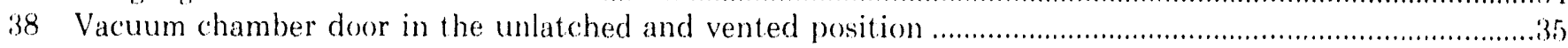

39 Source and detector configurations for calibration of the Model 5005-T'F ..............................................36

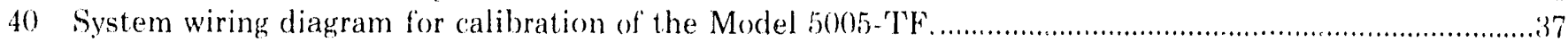

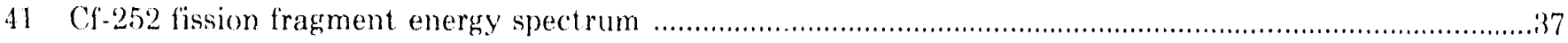

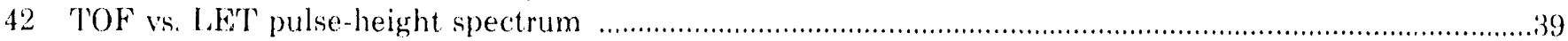

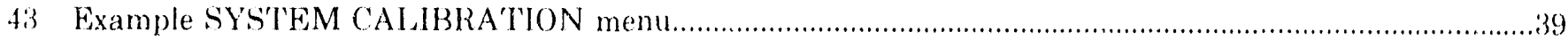

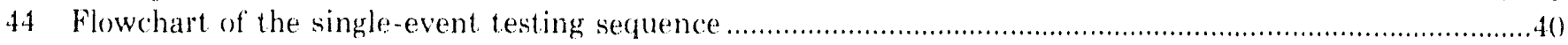

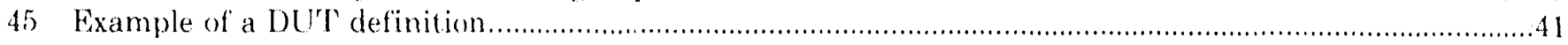

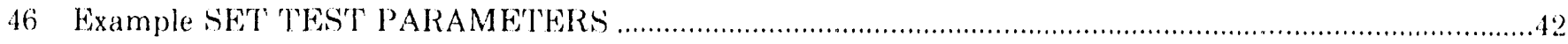

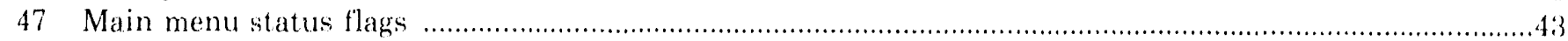

48 Cross section vs. LET' curve for the Harris HS-6514 RH SRAM ........................................................45

49 Illtra-thin-film scintillation detector light-pipe and holder ..............................................................46 


\section{Tables}

1 'The Lockheed Corporation's Estimate of the Cost of a Complete Single-Event

Characterization of a Radiation-Hardened Memory Device......................................................................... 9

2 Average Heavy- and Light-Fragment Characteristics 


\section{Advanced Development of the Spectrum Sciences Model 5005-TF, Single-Event Test Fixture}

\section{General Information}

This report summarizes the advanced development of the Spectrum Sciences Model 5005-TF, Single-Event Test Fixture. The Model 5005-TF uses a Californium-252 (Cf-252) fission-fragment source to test integrated circuits and other devices for the effects of single-event phenomena. Particle identification methods commonly used in high-energy physics research and nuclear engineering have been incorporated into the Model 5005-TF for estimating the particle charge, mass, and energy parameters. All single-event phenomena observed in a device under test. (DUT) are correlated with an identified fission fragment, and its linear energy transfer (LET) and range in the semiconductor material of the DUT.

The development of the Model 5005.TF was accomplished as a technology transfer activity. The Federal 'Technology Transfer Act of 1986 delegates authority to the directors of the national laboratories to enter into cooperative agreements with the private business sector. Commercialization of taxpayerfinanced technology is permitted under the BayhDole Act of 1980. This law permits small business ownership, subject to certain limitations, of new products and new processes developed under technology transfer agreements.

\subsection{Introduction}

Single-event testing of microelectronics at cyclo. tron and tandem Van de Graaff accelerators is very expensive. Depending on the number of test conditions (for example, number of parts to be tested, angle of incidence, number of ion beams used, temperature, bias, total dose, and decoupling resistance in hardened-static memories), the cost for a single-event characterization of a single-part type ranges from

" $\$ 10,000$ to $\$ 100,000$. For example, the cost of obtaining a simple cross section versus LET curve for a few samples of the Sandia SA.3240 CMOS SRAM was

typically $\$ 10,000$ for tests performed at the Berkeley 88-in. cyclotron. The total cost of the complete char- acterization of the SA3240 exceeded $\$ 100,000$. According to the Jet Propulsion Laboratory, the industry average ranges from $\$ 30,000$ to $\$ 50,000$ for a previously uncharacterized part type. ${ }^{1}$ Table 1 gives the Lockheed Corporation's current estimate of the cost of a complete single-event characterization of a radiation-hardened memory device. Single-event tests using Cf-252 fission fragments are much less expen. sive and more convenient than accelerator testing. However, it is necessary to address the risks associated with the range of LET and the limited depth of penetration of fission fragments in microelectronics materials.

\section{Table 1. The Lockheed Corporation's Estimate of the Cost of a Complete Single-Event Characterization of a Radiation-Hardened Memory Device}

BNL Tandem Facility at $\$ 425 / \mathrm{hr}$

reduced rate

$\$ 25,500$

Labor at $\$ 77 / \mathrm{hr}$ 24,486

Travel and expenses for two people

Equipment shipping expense

Total

$\$ \overline{56,126}$

The purpose of a test and evaluation activity is to detect defects in the article being tested. When test data are used in decision making, two types of error can occur. A Type I error is the incorrect conclusion that a defect has been detected. Type I errors impact project costs and schedules by convincing the program management that costly corrective action is required when no defect was really present. In general, it is important to guard against Type I errors if the parts being tested are expensive.

The failure to detect a defect is a Type 11 error. Type II errors reduce the reliability of satellite systems by convincing the program management that no defect exists when one will actually be seen in space. Most of the errors that can result from using fission fragments for single-event tests are 'Type II. They can 
be caused by the limited penetration depth of the fragments in microelectronics materials leading to false conclusions of single-event immunity, or underestimating the single -event rate in space by underestimating the cross section. They can also be caused by the rapid decrease in LET with depth of penetration, thereby overestimating the LET threshold and underestimating the single-event rate in space. Depthof-penetration issues are generally becoming less important in the new, integrated-circuit technologies.

The probability of making a Type II error can be reduced. Correctly estimating the thicknesses of the nonparticipating cover layers of the DUT, and the subsequent depth over which charge collection will occur (keeping in mind that conservative estimates may lead to Type I errors) can reduce error probability. Further, the impact of Type II errors may not be very significant in fault-tolerant systems. For such systems, Cf-252 fission-fragments may be used to provide the bulk, if not all, of the single-event susceptibility data required. The system design strategy would be to identify and eliminate most of the singleevent problems using Cf-252 tests. To rely on hardware and software fault tolerance would handle the rest. Such an approach is currently used in miniature satellites or "cheapsats."

In keeping with good engineering pr ctice, it is necessary to minimize the following:

$$
\begin{aligned}
\text { Total cost }= & \text { probability of a Type I error } \\
& \times \text { consequences of a Type I error } \\
& + \text { probability of a Type II error } \\
& \times \text { consequences of a Type II error } \\
& + \text { testing costs. }
\end{aligned}
$$

Single event testing using Cf-252 fission fragments as the radiation source will minimize the total cost of a test and evaluation activity. This radiation source will minimize costs if the consequences of Type I and Type II errors are not significant enough to merit the more expensive accelerator testing. For satellite systems in which the consequences of Type I and Type II errors are perceived to be very significant, Cf-252 testing as an initial parts screen before accelerator testing may still reduce the total cost.

Cf-252 testing can also minimize the total cost of developing single-event hardened microelectronics by providing a very convenient and low-cost method of characterizing process iterations. Here, the device process parameters are readily available for reducing the probabilities of Type I and Type II errors.

The Model 5005- TF can be purchased from Spectrum Sciences for $\$ 125,000$. The purchase price includes a turnkey system installed at the customer's site and training for the system users. This amount can easily be spent for characterizing a few part types at a cyclotron or tandem Van de (iraaff accelerator. The operating costs of the Model 5005-TF are insignificant compared to the $\$ 600 / \mathrm{hr}$ rate of accelerators. The Model 5005-TF may be co-located with automatic test equipment that is too large or too expensive to transport to an accelerator.

The principal disadvantage in operating the Model 5005-TF is the low-flux rates that must be used. (This is, however, a very good way to conduct dynamic tests of switched parts such as microprocessors.) Since the fission-fragment, parameters are correlated with single events, the DUT must be checked for single events after the arrival of each fission fragment and before the next fission fragment arrives. Thus a test time of a week (depending on the time it takes to examine the DUT for single events) may be required to accurately accumulate the cross section vs. LET curve. Thus, approximately 50 part types may be characterized per year with a single Model 5005-TF. This is, however, the equivalent of $\$ 500,000$ to $\$ 5,000,000$ of single-event testing at an accelerator.

\subsection{Purpose of the Model 5005-TF}

The Model 5005-TF can identify the Cf-252 fission fragment that causes a single event to occur in a DU'T. This is accomplished by a two-parameter method of particle identification. (A three-parameter method could also be implemented by the addition of another channel to the pulse-height ADC.) 'The surface, average effective, and exit LE'T values for a buried layer of semiconductor material are calculated for the user by the Model 5005- $\mathrm{TH}$ software. The Model 5005-TF' is designed to measure the LET' values with a precision of at least $1 \mathrm{MeV}-\mathrm{cm}^{2} / \mathrm{mg}$. The accuracy of the LET measurements will depend primarily on how well the user can specify the depth and thickness of the active buried layer. The total cross section vs. LET curves are displayed as the singleevent data accumulates.

\subsection{General Description} of

The Model 5005-TF, shown in Figure 1, consists

1. A borated polyethylene neutron shield.

2. A Cf-252 source holder and linear-motion adjustment.

3. Two photomultiplier tubes (PMT's) with ultra-thin scintillators $(1-\mu \mathrm{m}$-thick $B(; 0)$ plastic foils). 
4. A rotation and translation mechanism for mounting of the DUT.

5. An internal electrical connection vacuum feedthrough plate.

6. The particle identification electronics.
7. A vacuum controller.

8. A turbo-molecular pumping system.

Items 1 through 5 are housed in a vacuum chamber. An IBM PC-compatible data-acquisition system is also included.

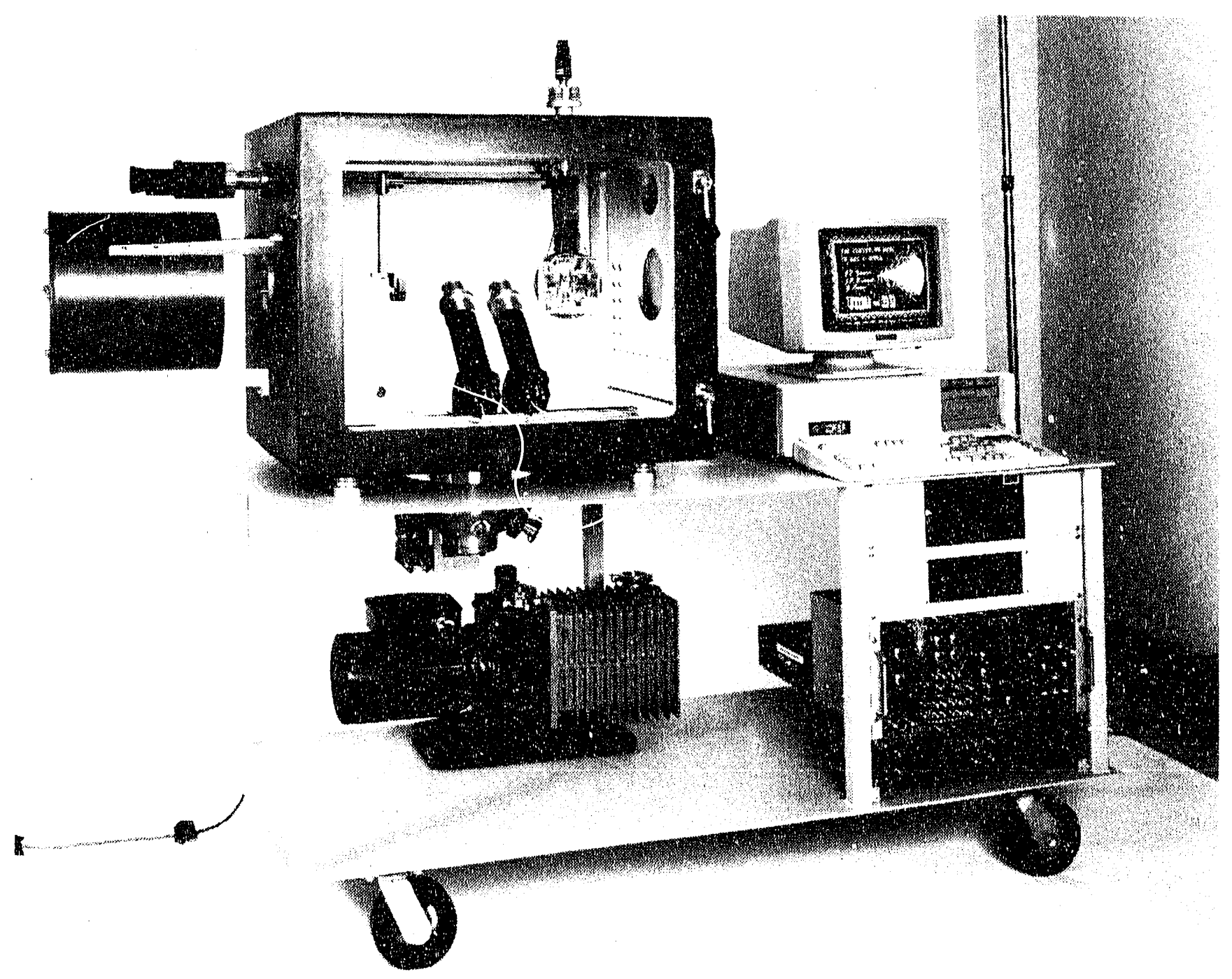

a. Photo

Figure 1. The Model 5005-TF Single-Event Test Fixture (continued) 


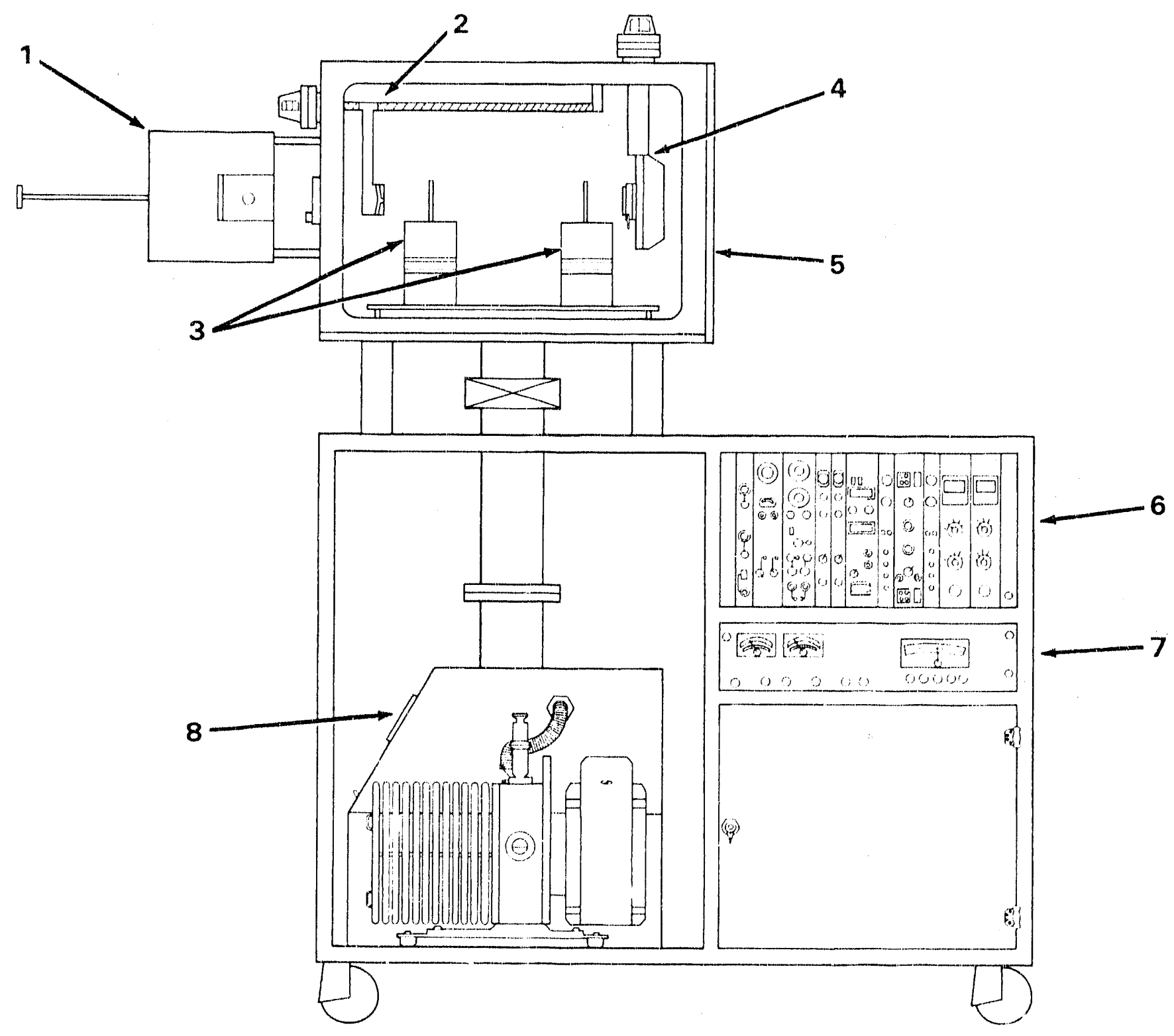

1. A borated polyethylene neutron shield.

2. A Cf-252 source holder and linear-motion adjustment.

3. Two photomultiplier tubes (PMTs) with ultra-thin scintillators $(1-\mu$ m-thick BC 400 plastic foils).

4. A rotation and translation mechanism for mounting of the DUT.

5. An internal electrical connection vacuum feedthrough plate.

6. The particle identification electronics.

7. A vacuum controller.

8. A turbo-molecular pumping system.

b. Drawing

Figure 1. Concluded. 
Wach Model 5005.'TH vacuum chamber is semicustom-designed to suit the user's requirements. The type of pumping system and the number of vacuum feedthroughs are just a few of the options. The turbo-molecular ,umping system can achieve high-vacuum levels in less than $10 \mathrm{~min}$. 'The DUT rotation and translation mechanism can position the DU'T for any possible angle of incidence (subject to depth-of'-penetration limitations). 'The source holder allows moving the source relative to the DU'T for separations of 10 to $80 \mathrm{~cm}$ (for the time-of-flight, TOF, measurements) down to $<5 \mathrm{~mm}$ (when a highflux rate is required). When the system is not in use, the Californium source is housed in the borated polyethylene shield. 'The entire system is mounted on a cart for relocation ease.

\subsection{Features and Specifications}

- Features

-Convenient and low-cost single-event test capability.

- Meets all the requirements of ASTM F1192. 88.

- Radiation safety designed into the system.

- Self-calibration feature.

- Precise particle identification.

- User-friendly software.

- Calculates the particle L.ET in buried layers.

- Displays the total device cross section vs. L.ET curve as the single-event data accumulates.

-. System is completely mobile.

-..- Dual axis DU'T rotation.

-... All DUT and source movements are external and can he performed under vacuum.

- Viewport for viewing the internal setup.

- Many user-selectable options such as the type and number of electrical feedthroughs.

- Specifications

- Dimensions: length 78 in., width 35 in., height $67 \mathrm{in.}$

- Vacuum chamber dimensions: length 48 in., width $333 \mathrm{in.}$, height $33 \mathrm{in}$.

- Total weight: $500 \mathrm{lb}$.

- Power requirements: 20 A, $120 \mathrm{Vac}$.

- Vent gas: dry nitrogen (optional).

--Ten-minute pump-down time.

- Vacuum levels to $55 \mathrm{E}-5$ torr.

Distance for TOFs: 10 to $40 \mathrm{~cm}$.

Scintillation foil thickness: $\sim 1 \mu \mathrm{m}$ (100 $\left.\mu \mathrm{g} / \mathrm{cm}^{2}\right)$.
-... Average energy attenuation by :cintillators: light fragment $7 "$, heavy fragment $9 "$.

- Efficiency of particle detection: $100^{\prime \prime}$ " for fission fragments, most alpha particles detected.

- Source activity: calibration $1 \mu \mathrm{Ci}$, test 5()$\mu \mathrm{Ci}$.

- Maximum fission fragment flux on DU'T (at $10 \mathrm{~cm}): 1000 / \mathrm{cm}^{2}-\mathrm{s}$.

- IBM PC DMA cycle time: <50 $4 \mathrm{~s}$.

-- Maximum dead time: 5 " ".

-- DUT angles of incidence: $90^{\circ} \pm 0.5^{\circ}$ (limited by DU'T packaging).

- DUT rotation in perpendicular plane: $360^{\circ}$.

- Maximum DU'T mounting-card size: 12 in. diagonal.

\subsection{Limitations}

The Model 5005-TF uses the wide range of ion species and particle energies produced by Cf-252 sources to characterize the single-event susceptibility of microelectronics devices. However, if the device tested does not upset when exposed to the fission fragments, then take care in drawing conclusions about single-event rates and use of the part in spacecraft.

The fission of $\mathrm{Cf}-252$ produces particles with energies $<1 \mathrm{MeV} / \mathrm{amu}$. With these nonrelativistic heavy ions, the thicknesses of the cover layers and charge collection volume must be estimated accurately to scale the $L E T$ as a function of the incident angle. Figures $2 \mathrm{a}$ and $2 \mathrm{~b}$ show the maximum averageeffective LFT at $0^{\circ}$ and $60^{\circ}$ incidence that can be achieved by the Model 5005-TF in silicon collection volumes 2 to $10 \mu \mathrm{m}$ thick, with passivation overlayers 2 to $10 \mu \mathrm{m}$ thick. 'These effective L.ET estimates include the energy attentuation by the gold encapsulation $\left(50 \mu \mathrm{g} / \mathrm{cm}^{2}\right)$ of large $\mathrm{Cf}-252$ sources, and the two $1-\mu \mathrm{m}$-thick scintillation foils.

Current Air Force goals for managing single-event upset (SEU) susceptibility in future satellite systems are three-fold: (1) the devices must not be vulnerable to upset by protons, (2) the SEU rate in the 10 ": worst-case geosynchronous onvironment must be <1E-7 errors per bit-day for , igh-speed devices of unspecified hardness, and (3) the SEU rate in the 10 ": worst-case geosynchronous environment must be $<1 \mathrm{E}-10$ errors per bit-day for special radiationhardened devices. 


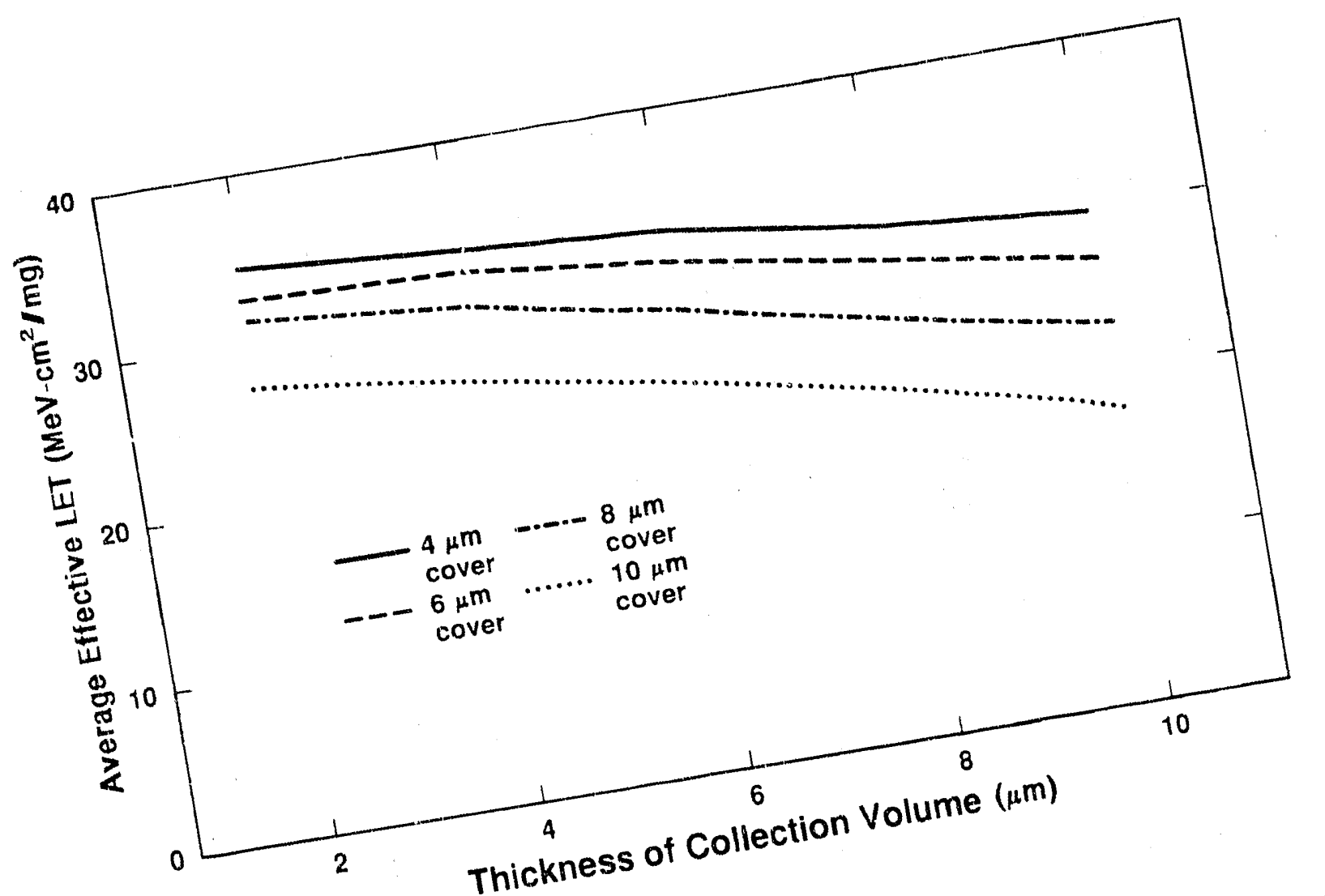

$x$

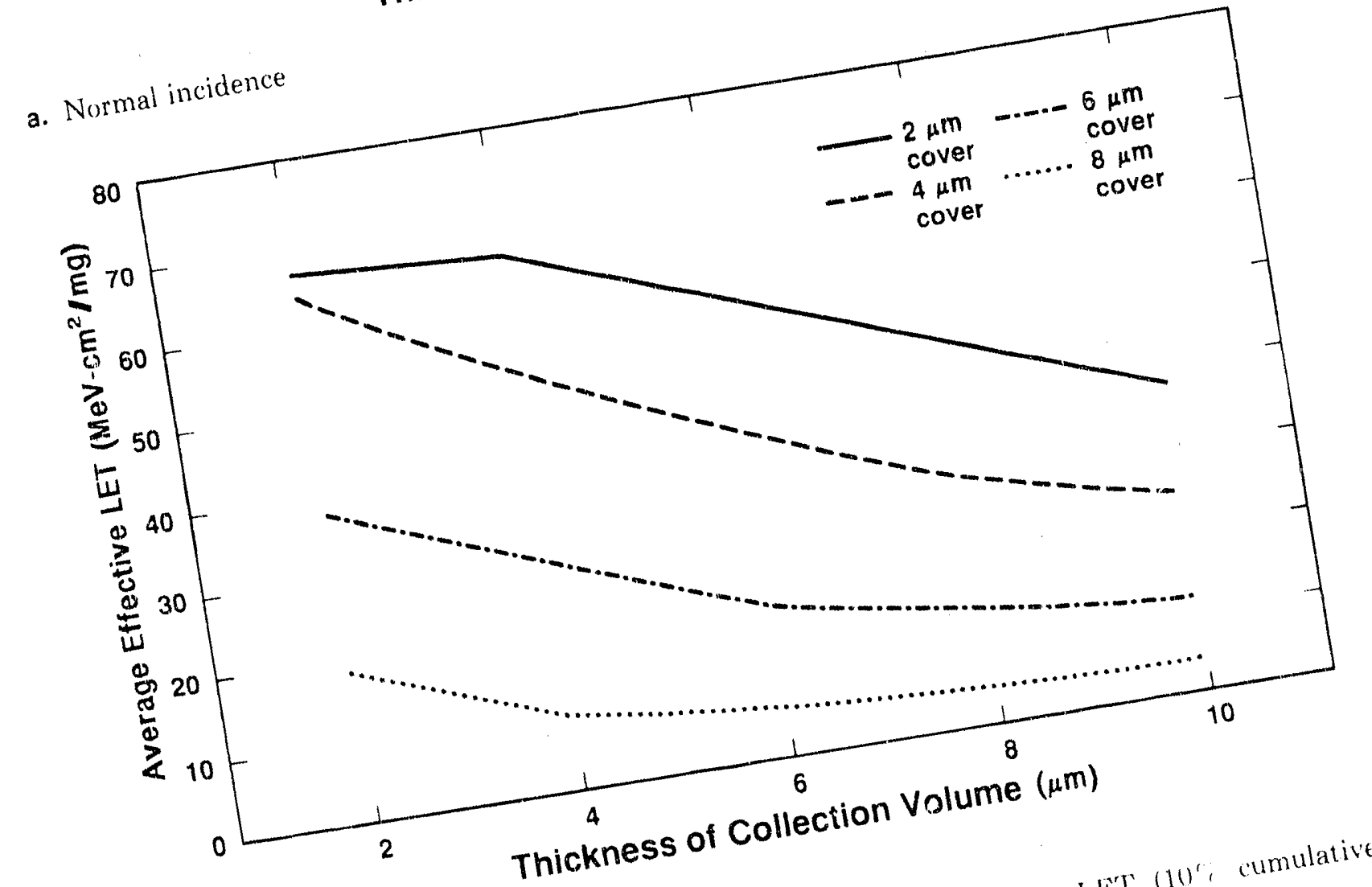

b. $60^{\circ}$ incidence

Figure 2. TRIM computer code estimat fraction in silicons for a DUT hured 
Protons do not directly produce upsets in microelectronics devices. They deposit energy via a nuclear interaction between the proton and a nucleus of the semiconductor material. When charged secondary particles created by the nuclear reaction pass through the collection volume, a single event can occur. The maximum LET a reaction product can have (a silicon ion) in silicon is $15 \mathrm{MeV}-\mathrm{cm}^{2} / \mathrm{mg}$. Therefore, if the device has a threshold LET greater than $15 \mathrm{MeV}-\mathrm{cm}^{2} /$ $\mathrm{mg}$, then the device will not be vulnerable to protoninduced single events.

Figure 3 shows the heavy-ion upset parameters (threshold LE'T vs, saturation cross section) taken by the Jet Propulsion Laboratory for bipolar devices. Data taken at the Los Alamos Ion-Beam Facility for recent MOSFET devices are also shown. No proton upsets are observed in these devices for LET thresholds $>8 \mathrm{MeV}-\mathrm{cm}^{2} / \mathrm{mg}$. Figure 2 shows that an average-effective $\mathrm{LET}$ of $15 \mathrm{MeV}-\mathrm{cm}^{2} / \mathrm{mg}$ can be obtained for all the combinations of cover layer and collection volume thicknesses. This average-effective
LE' is obtained by the selection of an appropriate angle of incidence and the use of degrading foils for thin devices. Therefore, the Model 5005-TF can be used to demonstrate the first Air Force goal for single-event hardness for these combinations of passivation and buried layer thicknesses.

The event rate in space is a function of the threshold LET and the saturation cross section of the microelectronic device. Figure 4 shows the upset rate in the $10 \%$ worst-case geosynchronous environment for a $100-\mu \mathrm{m}^{2} /$ bit cross section and a threshold LET equal to the maximum average-effective $\mathrm{LE}$ ' $\mathrm{T}$ given in Figure 2. As seen, the Model 5005-TF will meet the second Air Force goal ( $<1 \mathrm{E}-7$ errors per bit-day) with the appropriate choice of angle of incidence for all of the combinations of cover layer and collection volume thicknesses shown. If the per-bit cross section is $<100$ $\mu \mathrm{m}^{2}$, it is even possible to meet the third Air Force goal ( $<1 \mathrm{E}-10$ errors per bit-day) for devices that have thin cover layers and moderately thick collection volumes.

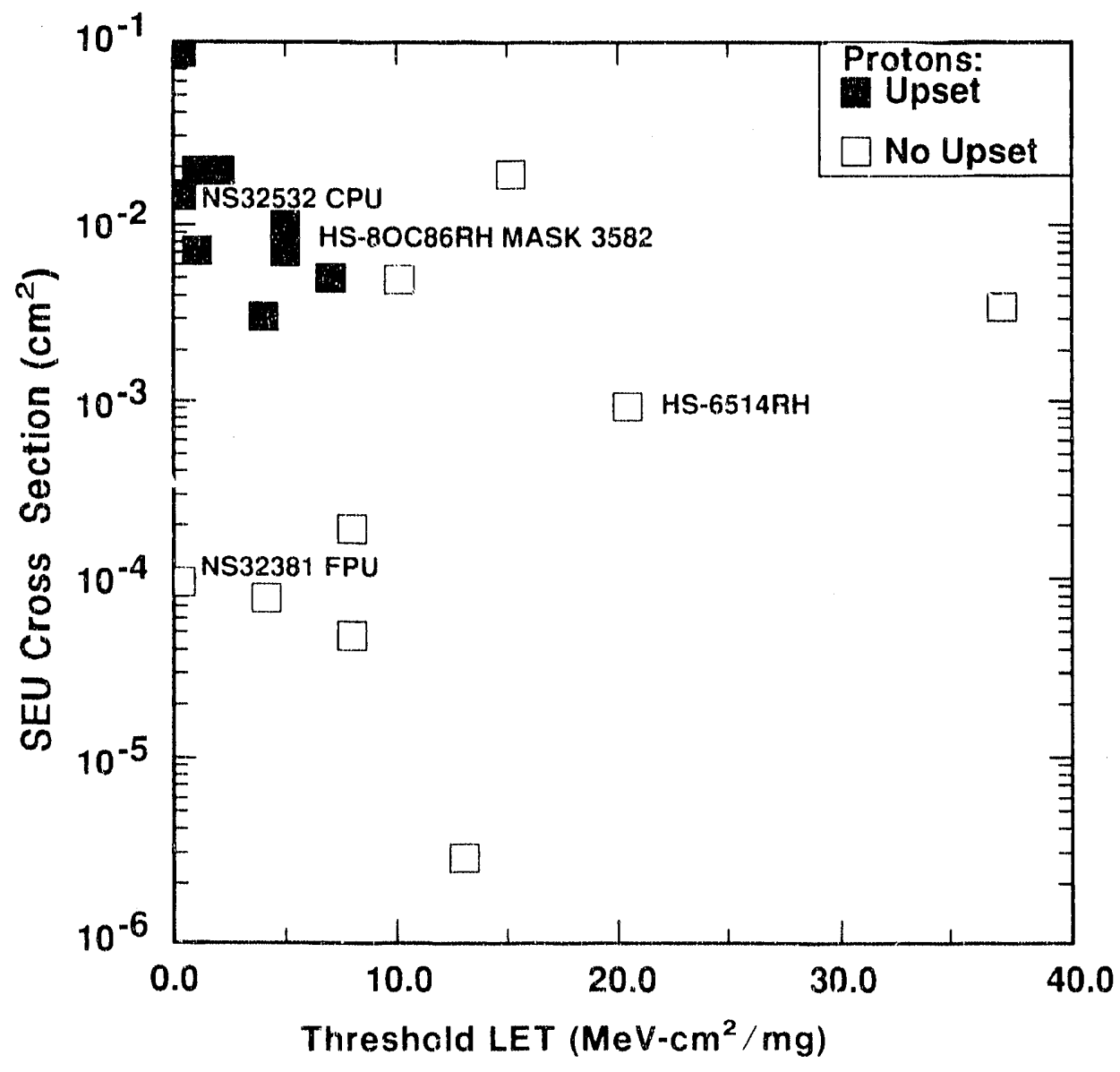

Figure 3. Comparison of heavy-ion to proton-induced singli-event upsets. 


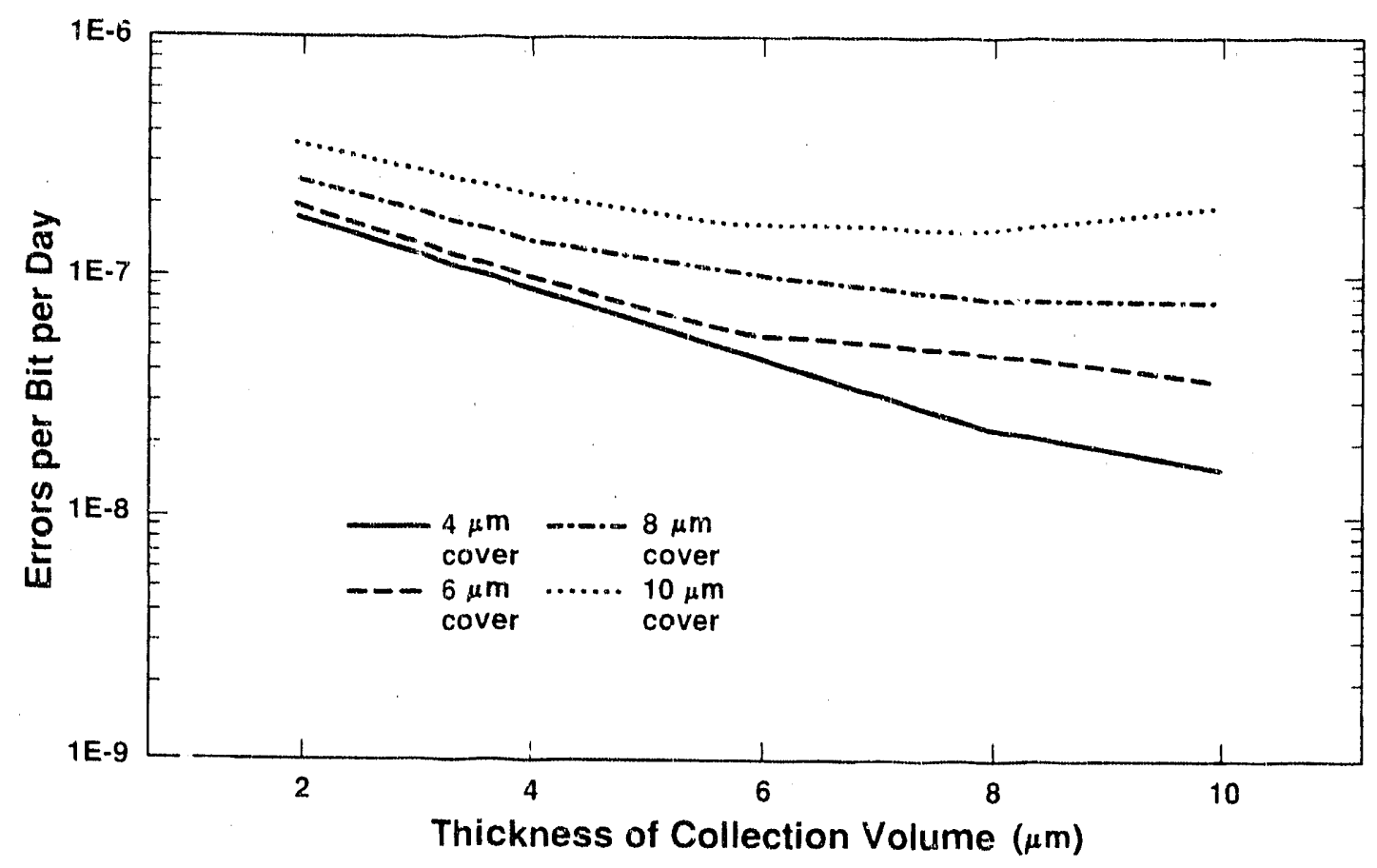

a. Normal incidence

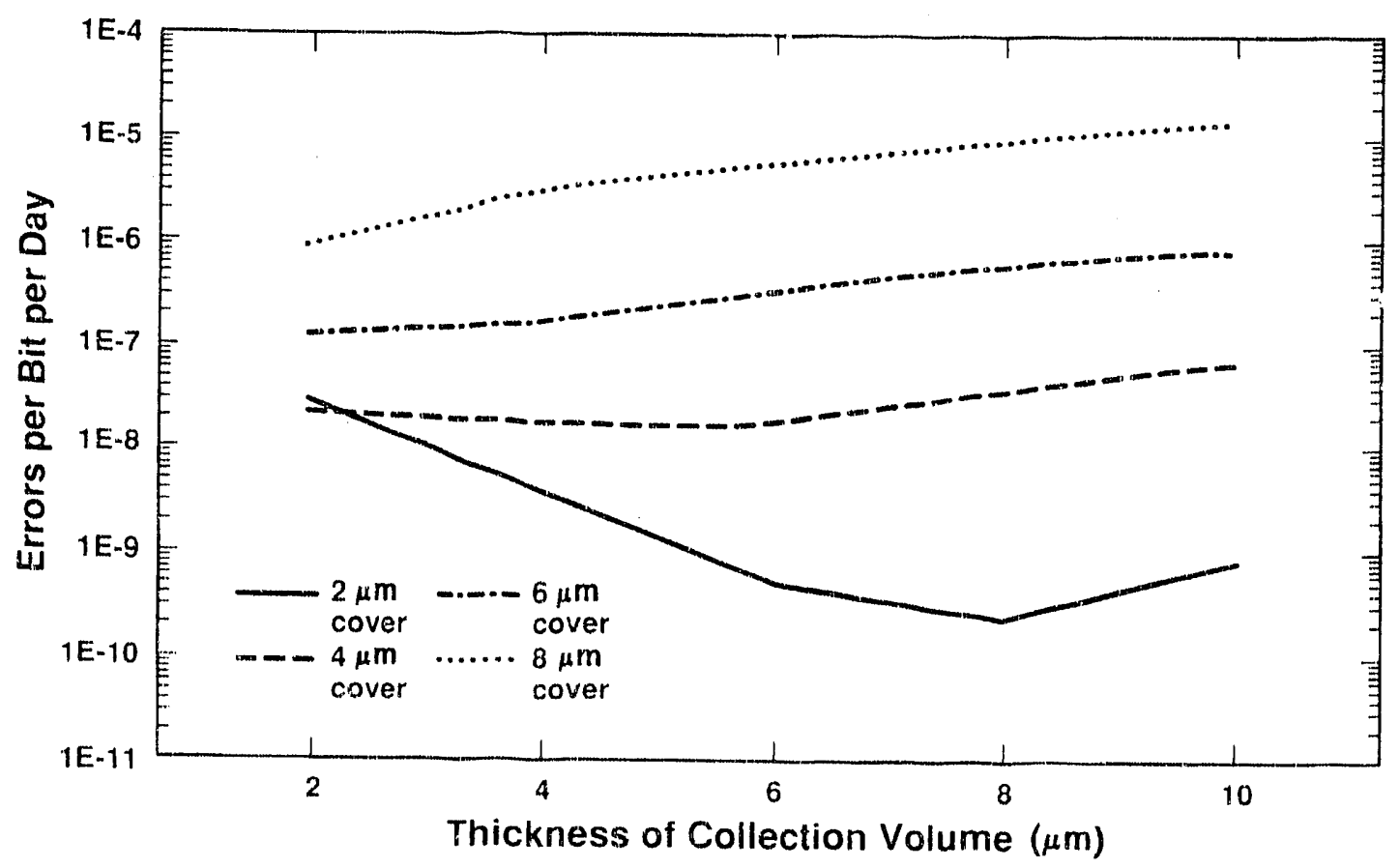

b. $60^{12}$ incidence

Figure 4. CREME computer code estimates of the upset rates for a $100-\mu \mathrm{m} \% /$ bit cross section and a threshold LET equal to the average-effertive LET given in Figure 2. The upset rates are estimated behind 60 mils of aluminum shielding in the 10 " " . worst-case cosmic-ray environment. 
The Air Force goals are worst case in the sense that a satellite may not need to survive the $10{ }^{\prime}$, worst-case geosinchronous environment. For exam. ple, the satellite may benefit from geomagnetic shielding and the earth's shadow in a low-altitude and low-inclination orbit. For swh an orbit, ons would expect the single-event rate specification to be less siressing than the 1E-7 errors per bit-day Air Force goal. Then a set of error rate upper bounds similar to Figure 4 would need to be calculated for the specific satellite environment to determine the suitability of the Model 5(j05-TF for piece-part qualification testing.

\section{Installation}

\subsection{General}

The Model 5005.'TF Single-Event Test Fixture should be installed by Spectrum Scienses. If the system is dismantled and relocated, the following is a brief synopsis on how to install the system.

\subsection{Unpacking}

The system is shipped in three crates and approximately four cardboard boxes. The largest crate houses the system cart and muclear instrumentation modules (NIM) electronics. The next size crate contains the vacum chamber, and the smallest crate contains the roughing pump. After inspecting all packages for damage.

1. Remove all items from containers (both crates and boxest, and

2. Inspect the contentis for damage.

If any item is damaged. contact Spectrum beiencess immediately.

\subsection{System Setup/and Preparation for Use}

The pictures in this section will assist you in the setup of the Model 5005-TF single Event Test Fixture.

\section{Vacuum Chamber.}

1. Place the rhamber on the system cart and position the feet o line up with the four holes in the top of the cart.

2. Secure the chamber to the cart with the $3 / 8-16$ bolts.

\section{Pumping System.}

1. Pace the turbo pump with the guard screen/ O-ring (Figure 51 between the pump and the bottom of the chamber.

2. Secure with the half-clamps and buits. Do not tighten the clamps yet.

3. Place the roughing yump on the bottom shelf of the systen cart if the exhaust port on the pump is vented to an outside exhaust hood.

4. If the oil-mist eliminator is used, then place the pump on the floor next to the system.

5. Whether the pump is located on the cart or the floor, position the silver flange on the turbo pump to face the roughing pump.

6. Secure the turbo-pump clamps starting with one side, then move to the opposite side.

7. Continue this procedure until the pump is evenly secured to the chamber.

8. (lsing the 40-in. flexible hose (Figure 6) connect the turbo pump to the roughing pump with the O-rings and clamps. The pumping system is now installed.

9. Refer to the Alcatel Vacuum Products owner's manual for additional instructions.

10. Now connect the cables from the turbo pump to the rack-mounted controller (Figure 7).

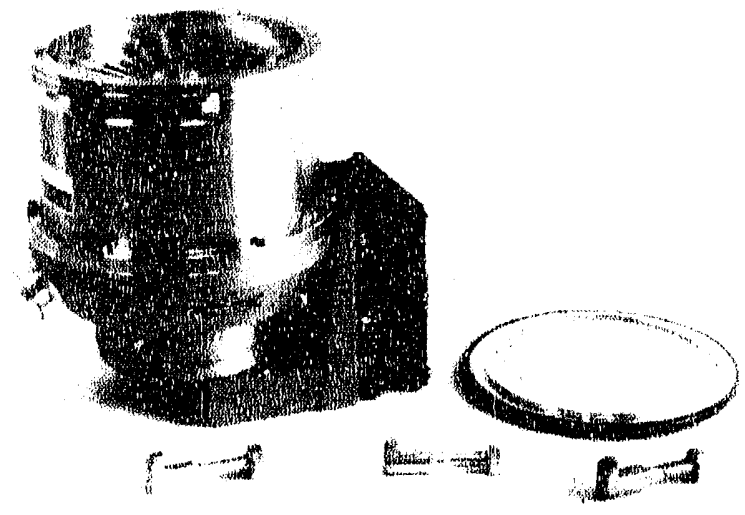

Figure 5. Alcatel Model 5400 turbo pump with the guard sureen/O-ring seal and half-clamps 


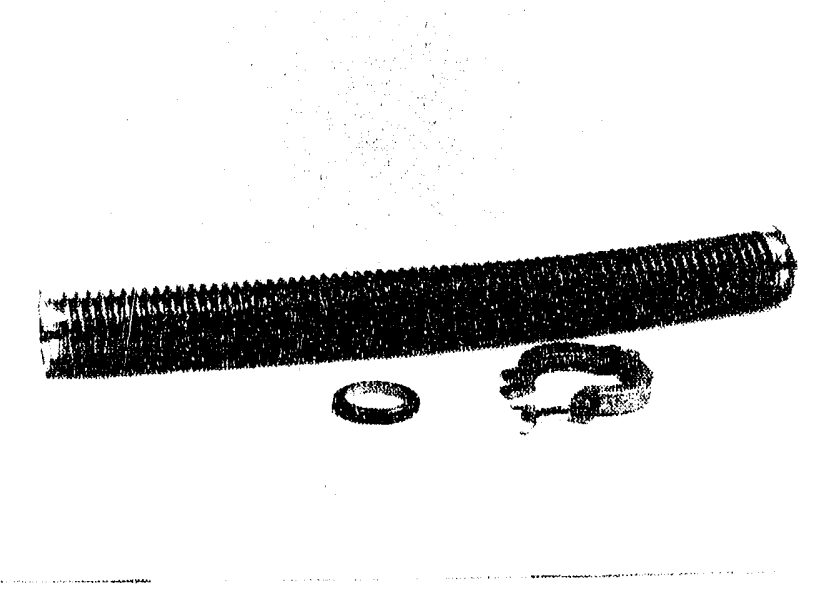

Figure 6. Flexible hose (40 in. long) used to comnec. the turbo pump to the roughing pump

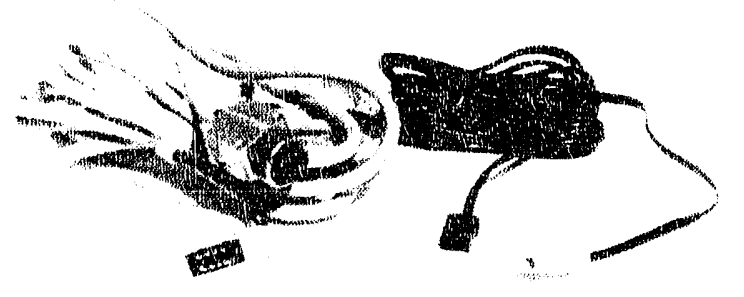

Figure 7. Cahles used to conned the turbo pump to the rack-molunted controller

Vacuum Gauges. The (iranville Philips (cil) vacuum-gauge controller should already be mounted in the system cart rack.

1. Install the convection tube and the ion gauge (Figure $x$ ) on the lltra Torr fitcings located on the back of the chamber (Figure 9).

2. Tighten the fittings so ther will not leak.

3. Install the cabling (Figure 10) from the vacuum gauges to the back of the ciP controller.

4. Refer to the (il) owner's manual for the proper procedure.
5. Do not forget to comnect the green ground wire to one of the feet on the bottom of the chamber.

Figure 11 shows the vacuum gauges properly installed and cabled.

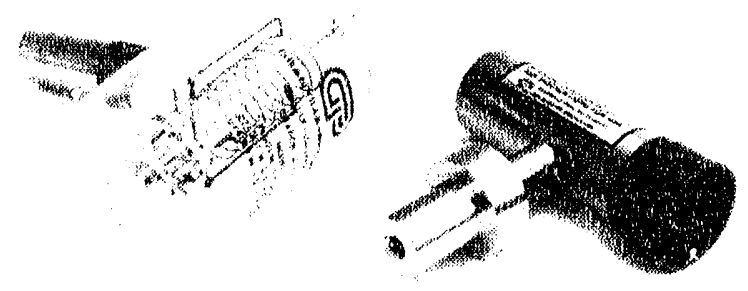

Figure 8. Vacuum gauges. (a) Comvectron tube (right) and ion gauge (left) (h) cables

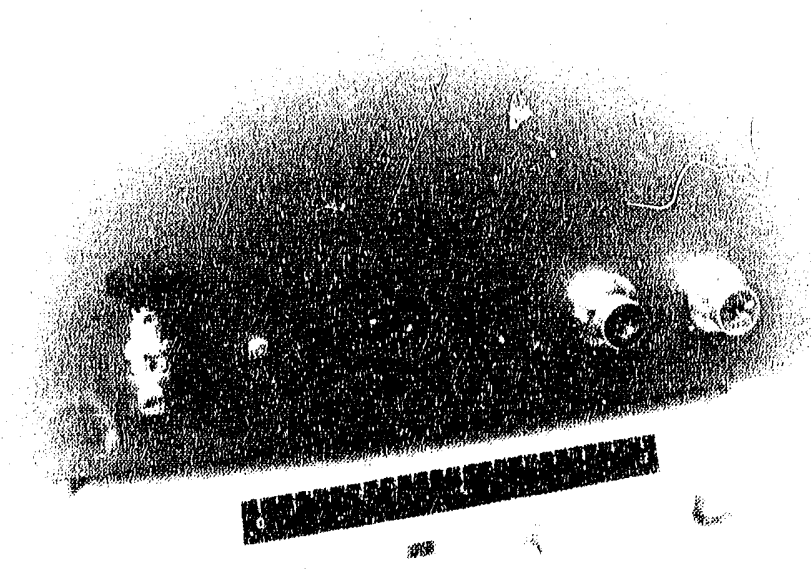

Figure 9. Ultra Torr tittings on the hack side of the vacuum chamber (right) 


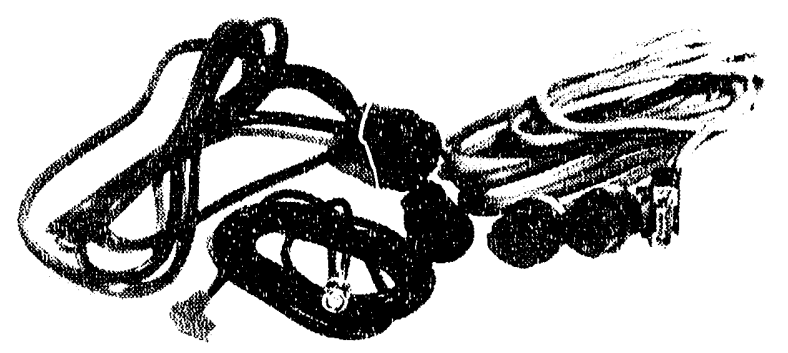

Figure 10. Cables for vacuum gauges

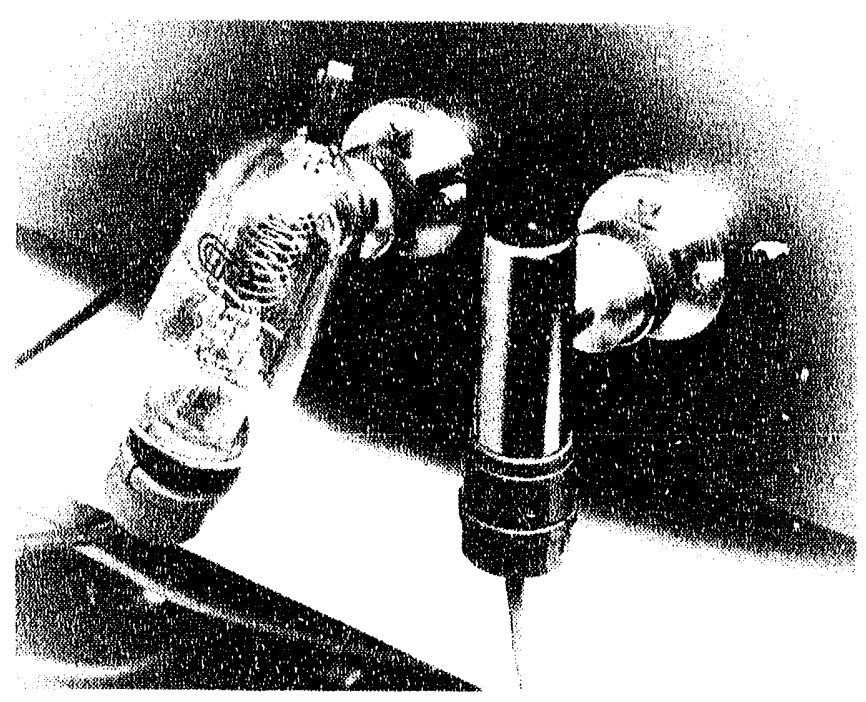

Figure 11. Convectron tube and ion gauge, installed and cabled

Photomultiplier Tubes and Scintillators. The photomultiplier tubes (PMTs) are held by a clamp holder and mounted on a slide track allowing the distance between them to be varied (Figure 12).
1. Loosen the clamps with an Allen wrench and slide the tubes into their holders.

2. Now place the scintillators on the top of the PMTs. (Use a small amount of optical grease between the PMT glass surface and the ultraviolet (UV)-transmitting lucite plastic to achieve a good optical connection.)

3. Position the PM'T in the holder so the source holder, the scintillators, and the DU'T mount are all in alignment.

4. Tighten the clamps on the PM'T's just enough to hold them securely-do not overtighten!

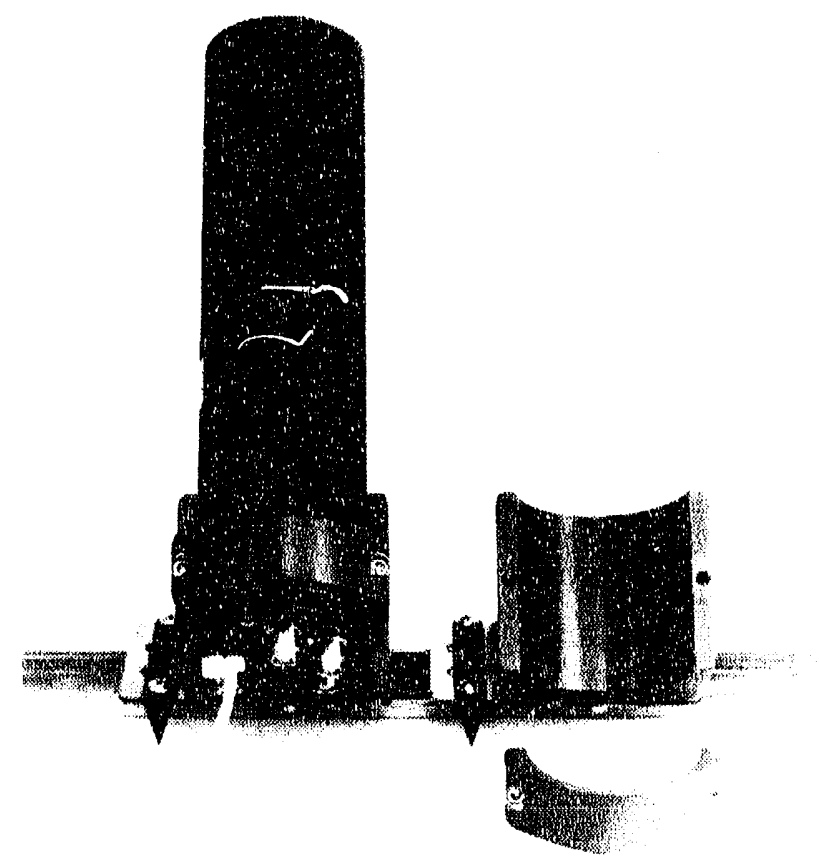

Figure 12. Clamp holder and slide rack used to position the photomultiplier tubes

Cables. Cabling the system is the last step. All coax cables supplied with the system are labeled with tie-wrap tags and should be the proper length. Refer to the "system wiring diagram" (Figure 13) for assistance in cabling the NIM electronics. 


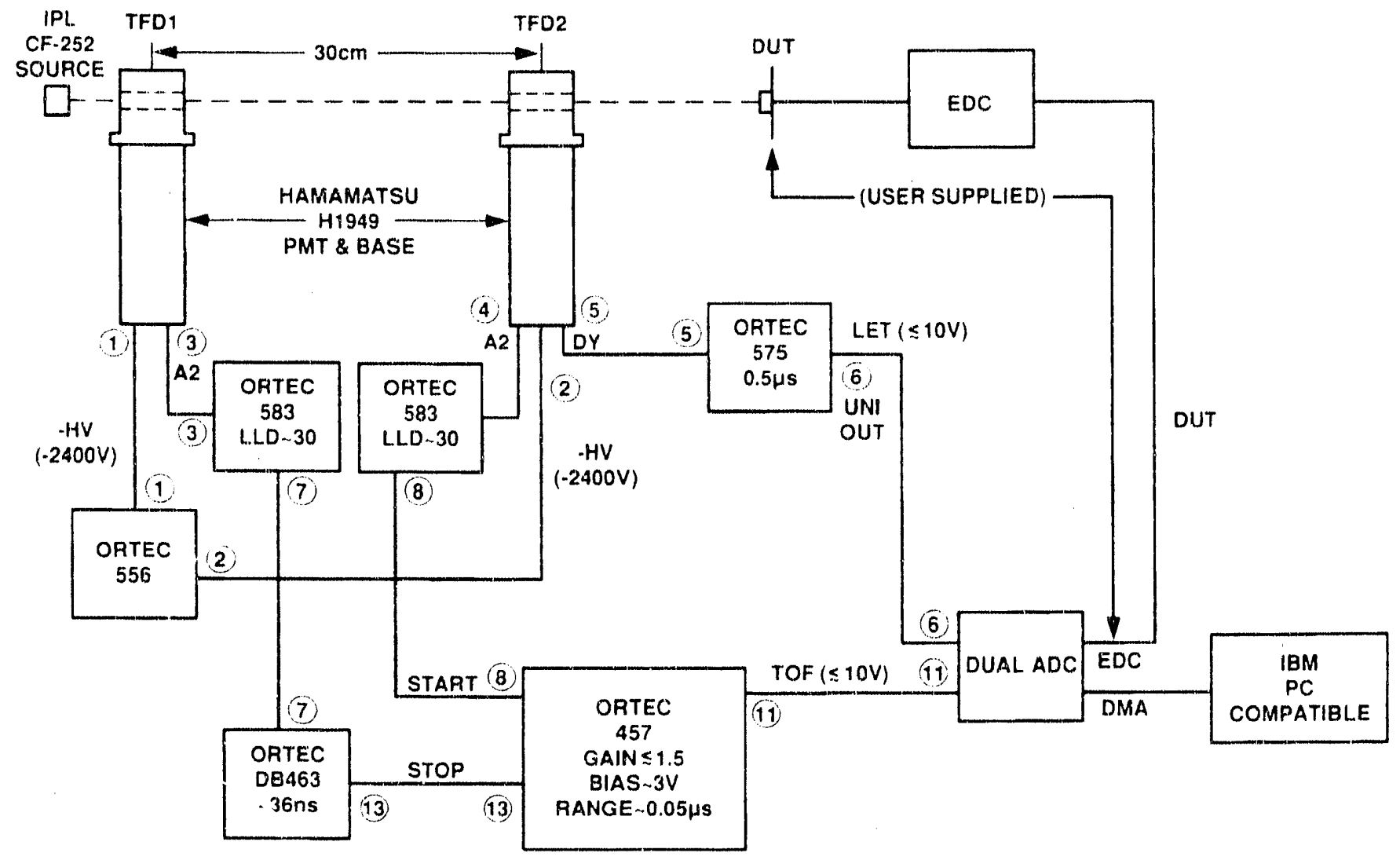

Figure 13. System wiring diagram. The NIM settings are for a $30-\mathrm{cm}$ flight path.

\subsection{Installation of the $\mathrm{Cf}-252$ Sources}

The two sources supplied with the Model 5005-TF are referred to as the "small" or "calibration" source and the "large" or "test" source. The calibration source has a specific activity of $-1 \mu \mathrm{Ci}$ and is housed in an inmodified IPL Model FF-252 holder. The test source has a specific activity of $\sim 50 \mu \mathrm{Ci}$ and is housed in a modified IPL holder. The modified holder has a 1/4-20 threaded hole in one end and has small "wings" on the sides for locking it into the source holder (Figures 14a and 14b). These modifications permit mounting of the large test source inside the vacuum chamber or removing for storage, without handling. The procedures for installing the calibration and test sources are as follows. 


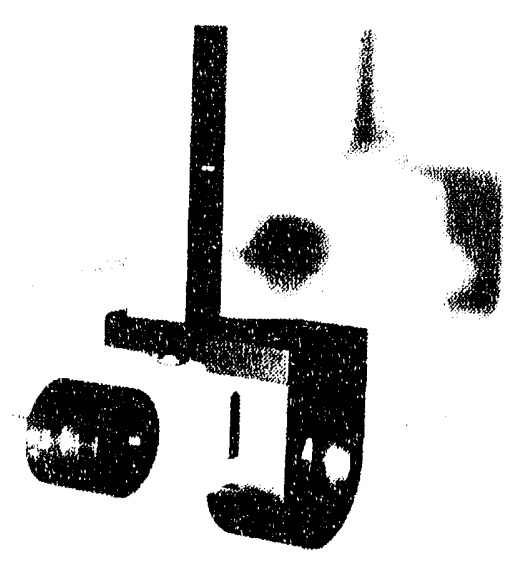

a. Cf-252 source and source holder

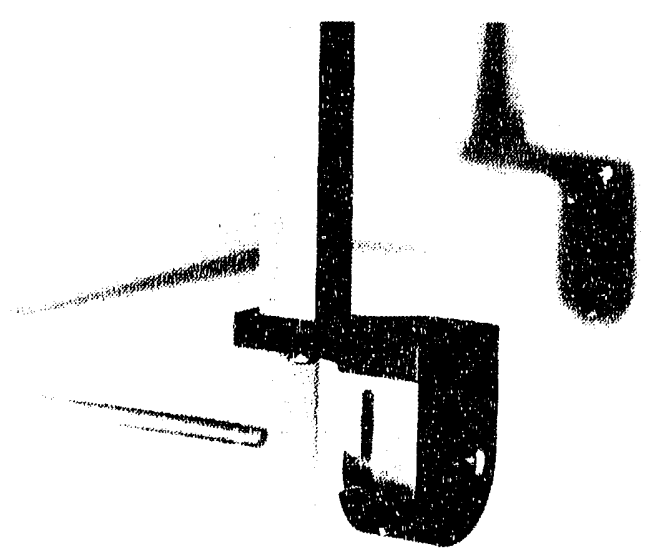

b. Cf -252 source installed in the source holder

Figure 14. Large Cf-252 source
Calibration Source Installation. The Model 501)- TF must be calibrated before use, and must be recalibrated anytime that the large test source or the scintillation foils are replaced. The small calibration source is used in the first step of the system calibration procedure (Section 4.3). 'To install the catihration source

1. Vent the vacuum thamber.

2. Using the standa'd operating procedures for your facility (see Appendix A for an example), remove the calibration source from its storage container.

3. Place the source in the source holder with the beveled hole facing the semiconductor nuclear particle detector.

4. Move the source into position using the linear movement feedthrough.

The system is now ready for pumpdown and calibration.

Test Source Installation. 'The large test source must. be removed from the shipping container and mounted on the 1/4-in. threaded rod before it can be used for system calibration and single event testing. Follow the standard operation procedures at your facilitv for handling the source, and perform the following steps quickly to minimize your exposure to the radiation ernitted by the source.

1. Open the source-access shutter valve and insert the $1 / 4$-in. threaded rod into the chamber (Figure $15 a$ ).

2. Screw the test source onto the rod (Figure 15h).

3. Insert the 1/4-in. rod (Figure 15c) into the source holder in such a manner that the "wings" of the test source mate with the slots in the holder.

4. Unscrew the $1 / 4-i n$. rod so that the wings lock into the source holder.

5. Extract the $1 / 4$-in. rod and close the sourceaccess shutter valve.

The system is now ready for pumpdown. The source is removed by venting the vacuum chamber and reversing the above instructions. 

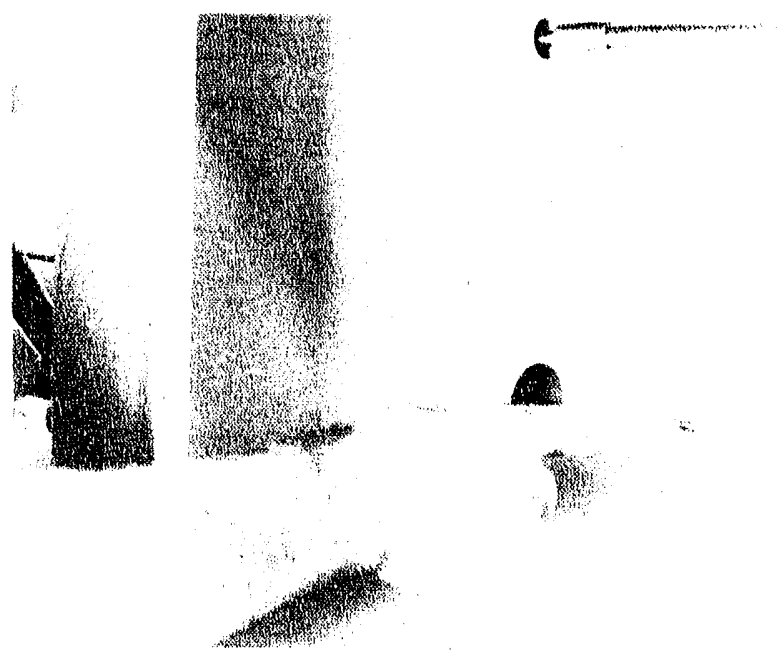

a. Threaded rod inserted into the vacuum chamber through the source-access valve

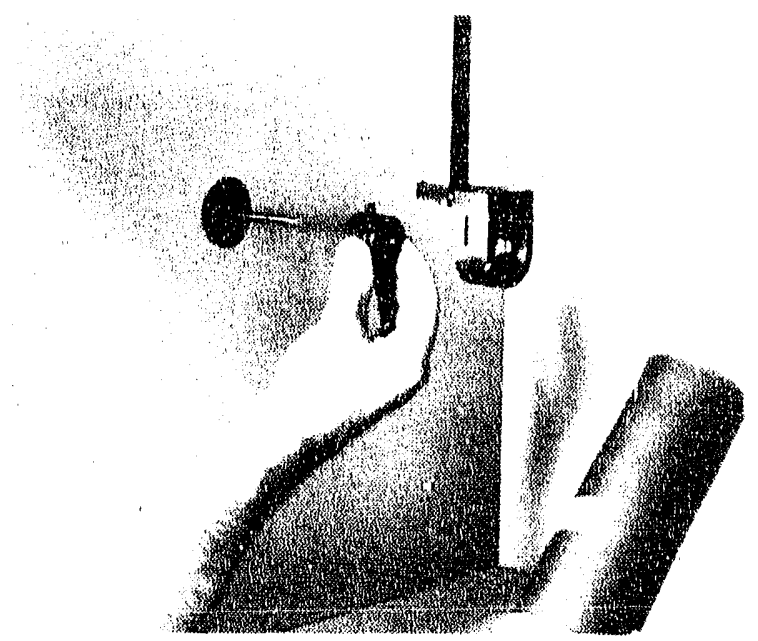

b. Large (1.252 source mounted on the threaded rod

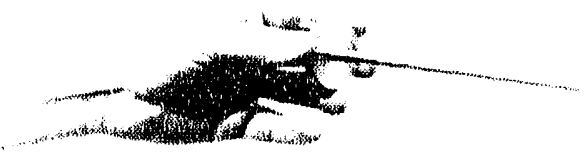

c. Threaded rod used to move the large (F-25i2 source between the sonrce holder inside the vacuum chamber and the borated polyethylene neut ron shield

Figure 15. Threaded rod used to position the large (f) 252 sources

\subsection{Installation of the IBM PC Software}

The Model 50)5-TF soltware is provided on several diskettes. ('The number of diskettes depends on the type of diskette requested.) For proper operation of the Model 5005-TF, the serial number of the software must match the serial number on the rear panel of the dual-channel pulse-height $\mathrm{AI})($ unit. Check the serial numbers to verify that you have received the correct software.

It is recommended that your IBM P( have a 2()-Mbyte hard disk and $640 \mathrm{~K}$ of random-access memory. Use the CHKDSK DOS command to determine how much space remains on the hard disk and how much memory is available on your PC. 'The $640 \mathrm{~K}$ of memory is recommended to accommodate changes that may be made to the software by spectrum Sciences from time to time. A minimum of 2()-Mbyte disk storage is recommended to store the large data files you will accumulate from single-event testing.

1. From the root directory on the hard disk create a directory by executing the IOOS command "MI) $\backslash 5005 \mathrm{~T} \mathrm{TF}$.

2. Execute "(D) $\backslash 5005 \mathrm{TF}$ " to change to this directory.

3. Now copy the contents of each diskette supplied with the Model 5005-TF' into the directory by placing the diskette in diskette drive A: and executing "COPY A: $\backslash *$.." (II your diskette drive is not $A$ : then use the appropriate diskette-drive letter designation.)

4. View the contents of the README. S'T file to obtain additional information about the Model 50(0)5-TF.

\section{System Components}

\subsection{Pumping System}

'The Model 5005-TH' pumping system consists of an Alcatel Model 5400 turbo-molecular pump and controller, and a Model 2013:3 roughing pump (Figures 5 and 16). The Models 5400 and 2013:3 have a pumping speed of $400 \mathrm{l} / \mathrm{sec}$ and $76 \mathrm{i} / \mathrm{l} / \mathrm{min}$, respectively. For all operation and maintenance procedures. consult the Alcatel manuals.

The system vacuum level is monitored by a (iP Series 300 vacuum-gauge cont roller (Figure 8 ). The controller is located in the rack mount on the sy'stem cart. An ion tube and convection gauge are installed on the back of the chamber and are interfaced to the 
vacuum system with Ultra Torr quick connectors. Refer to the GP owner's manual for more information.

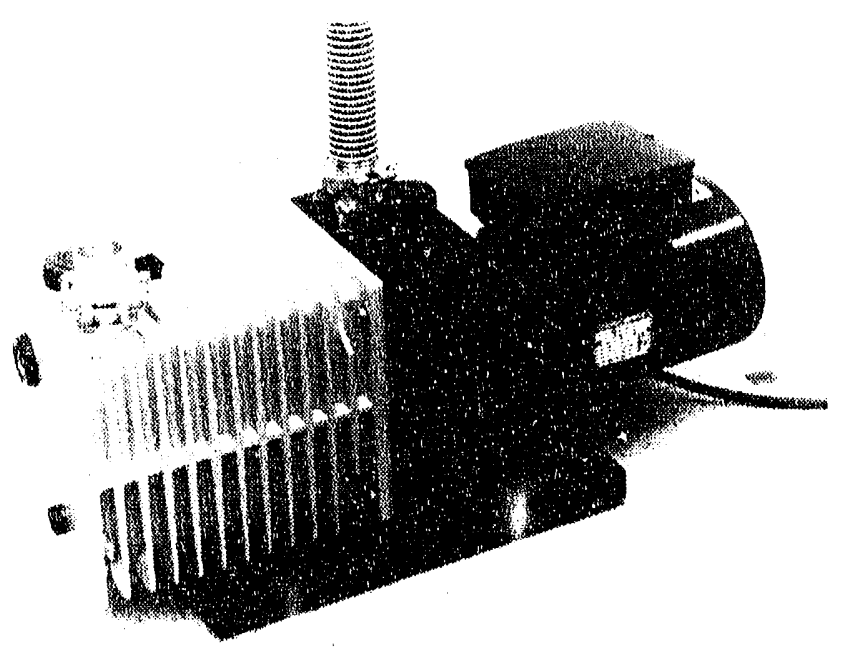

Figure 16. Alcatel Model 2033 roughing pump

\subsection{Vacuum Chamber}

The Model 5005-TF vacuum chamber is constructed of 0.625 -in. aluminum and is anodized for cosmetic purposes. (It is necessary that the inside of the chamber not be anodized to ensure a good vacuum.) An ultimate pressure of $<5 \mathrm{E}-5$ torr is obtainable; however, the system can be operated at pressures as high as $2 \mathrm{E}-4$ torr. 'This pressure must be achieved before the PMT bias is applied (a higher pressure will cause the PM'Ts to arc and will caluse permanent damage to the tube-base circuit). The rotary feedthrough on the left side of the chamber (Figure 17) is the linear movement of the source to the DUT' that controls the particle flux. The 3-digit readout on the feedthrough will give the distance from the DU'T in centimeters. The shutter just below the feed through is used to insert the source into the chamber and secure it in the source holder. When the system is not in use, the source is housed in a bora $\mathrm{d}$ polyethylene neutron shield (Figure 18). The source is on the end of a threaded 1/4-in.-dia rod.

The feedthrough on the top of the chamber is the I)U'T-positioning (angle and rotation) mechanism (Figure 19). The DU'T' is mounted on the face of the round mounting bracket inside the chamber (Figrure 20). Always mount the IUT so that it is flush and in the center of the mounting base. This will ensure that the fission fragments are always incident on the DUT', regardless of its orientation. The 3-digit counter on the top end of the feedthrough translates into degrees of rotation. The flange on the feedthrough gives the angle at which the particles are striking the DU'T, On the back side of the chamber are the Ultra Torr fittings for the vacuum-controller gauges and the vent valve (Figure 21).

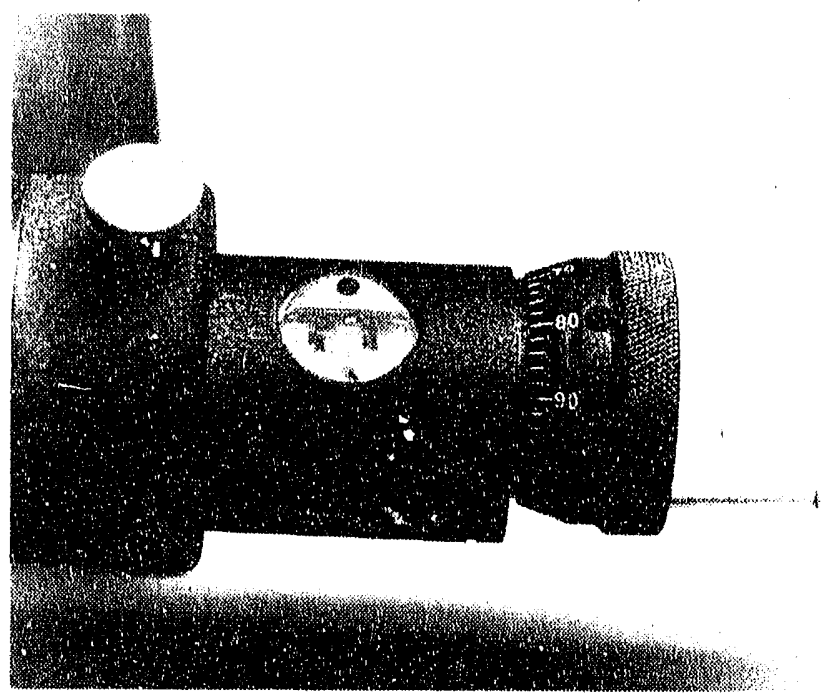

Figure 17. Rotary feedthrough used to control the particle flux

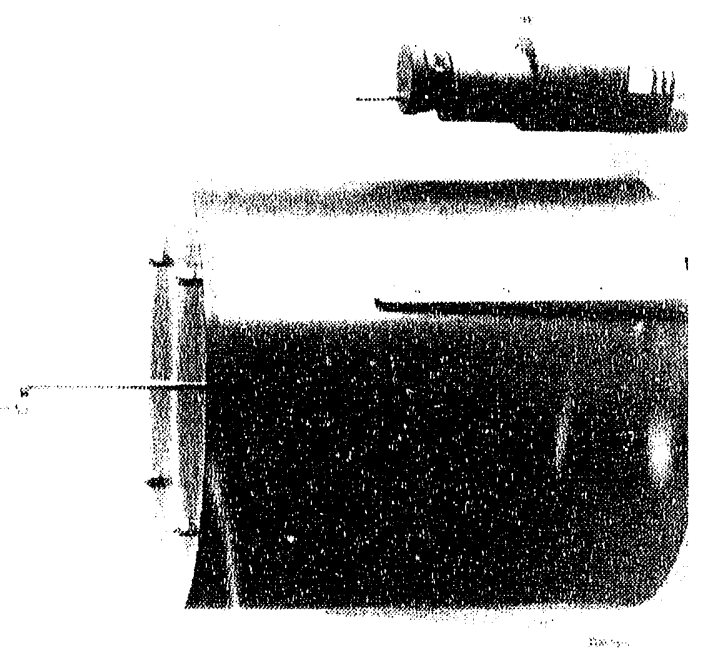

Figure 18. Borated polyeth lene neutron shield used to store the large (f-252 source when it is not in use 


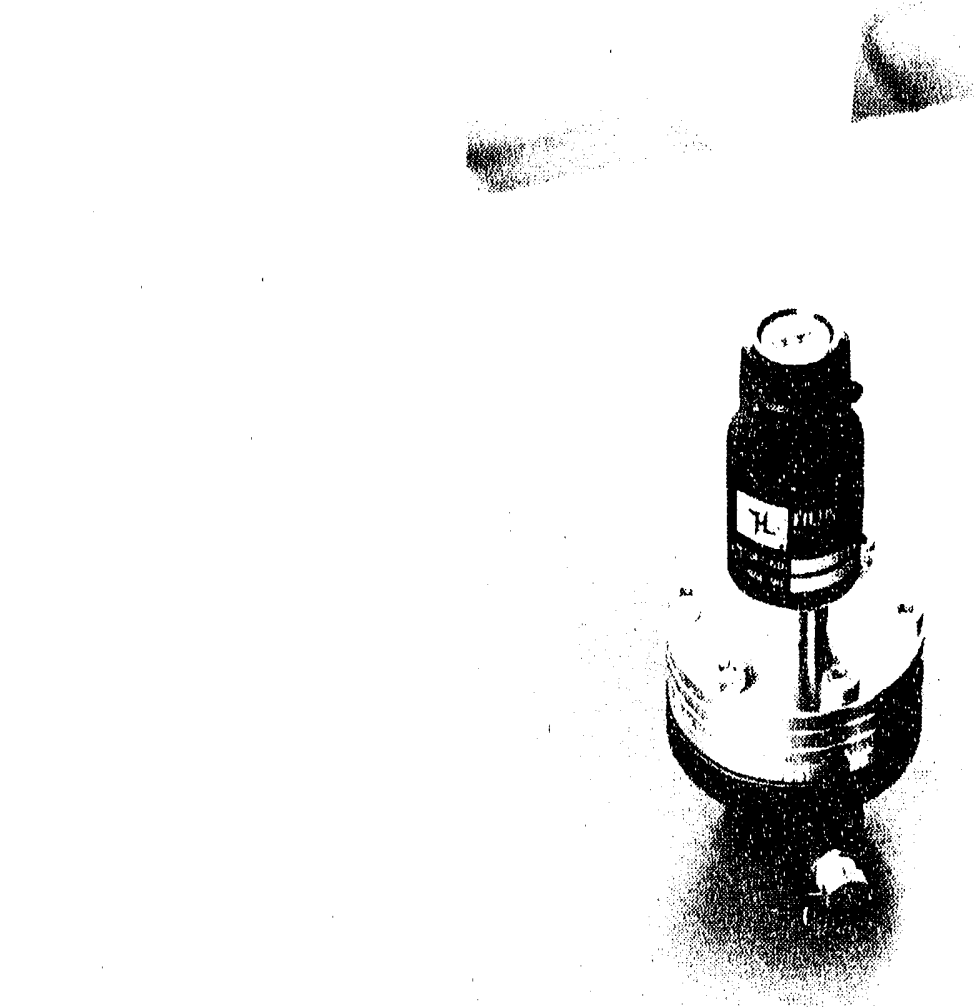

Figure 19. Angle and rotation feedthrough for positioning the device under test.
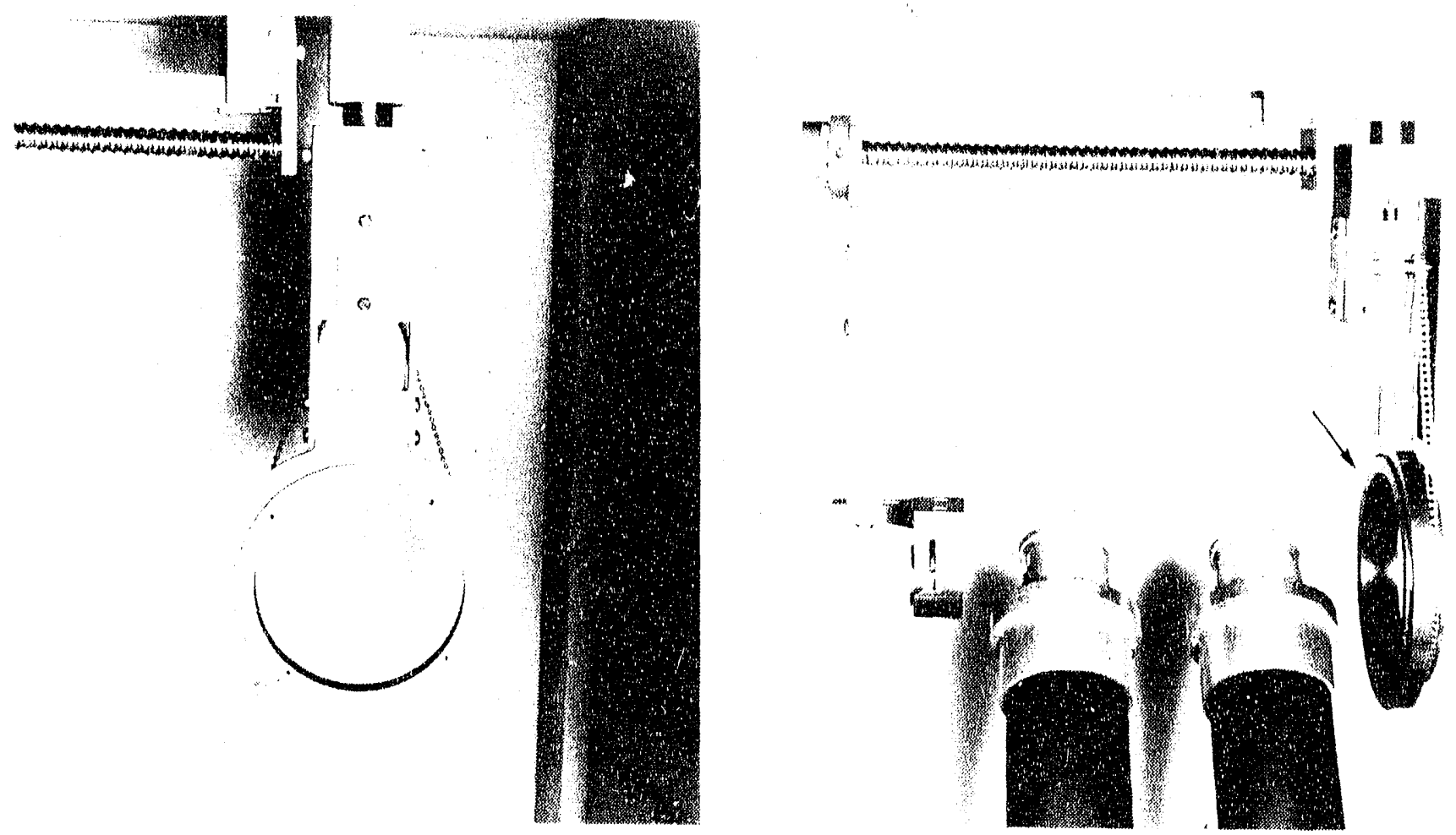

a. Downstream view

b. Side view

Figure 20. Fixture for mounting the device under test inside the vacuum chamber 


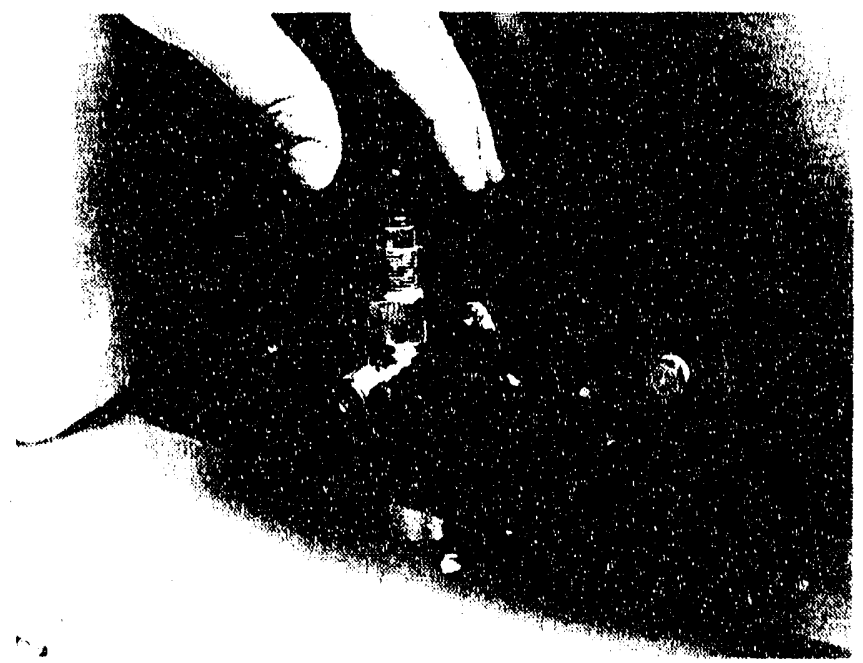

Figure 21. Vent valve on the back side of the vacuum chamber

\subsection{Particle Detectors and C $\$-252$ Sources}

The Model 5005-TF Single-Event Test Fixture includes two types of particle detectors: an ultrathin-film scintillation detector and a semisonductor nuclear-particle detector (NPD) (Figures 2.2 and 23). The thin-film detector (TFD) is used for the TOF and PMT pulse-height measurements. The TFD consists of the PMT base unit, a scintillator holder and light pipe, and a $1-\mu \mathrm{m}$-thick $\mathrm{BC} 400$ scintillation plastic foil. The foil is sandwiched between two pieces of UV-transmitting lucite (Figure 24). The light-pipe assembly is inseried into an aluminum holder and held in place by a small Allen headset screw (Figure 25). The aluminum housing is mounted on the PMT and base unit so that the light pipe mates with the glass surface of the PMT. A slight modification has been made to the PMT base (ac-coupling) and the circuit diagram in Figure 26 shows how this was done. The $1-\mu \mathrm{m}$-thick $\mathrm{BC} 400$ scintillators have a $100 \%$ detection efficiency to fission fragments when used with the Hamamatsu H1949 PMT (as determined by coincidence counting with the NPD).

The NPD is used to calibrate the Model 5005-TF. Two Cf-252 sources are used: a $1-\mu \mathrm{Ci}$ calibration source and a $50-\mu \mathrm{Ci}$ source for single-event testing. The smaller $1-\mu \mathrm{Ci}$ source is needed because the large $50-\mu \mathrm{Ci}$ source is covered with a $50-\mu \mathrm{g} / \mathrm{cm}^{2}$ layer of gold to prevent the self-transference of the Cf-252 material onto other surfaces inside the Model 5005-TF vacuum chamber. The $1-\mu \mathrm{Ci}$ source has a small enough specific acti:ity in which $\mathrm{Cf}-252$ contamination is very unlikely, and no gold cover layer is needed. Thus, the small source provides an unattenuated fission-fragment energy spectrum for energy calibration of the NPD. The NPD is mounted in the middle of the DUT sample holder using 6-32 screws (Figure 20). Finally, the $1-\mu \mathrm{Ci}$ source is placed in the source holder with the PMT and the scintillators in place.

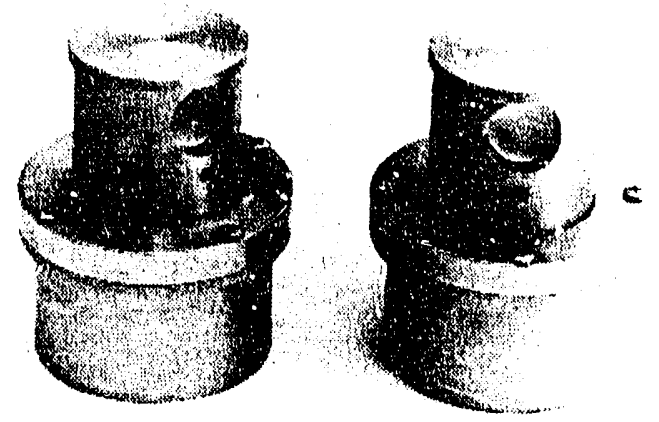

Figure 22. Ultra-thin-film scintillation detectors

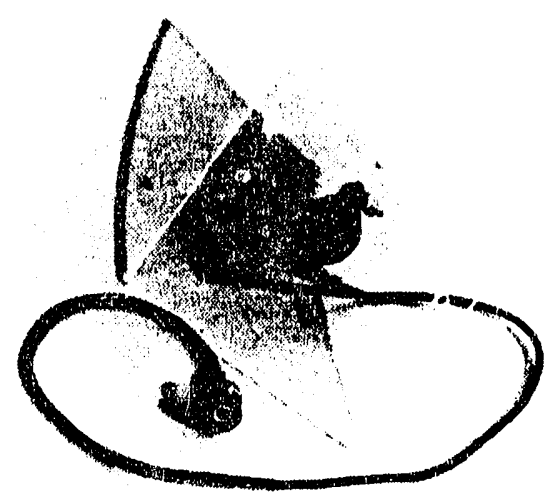

Figure 23. Semiconductor nuclear particle detector 


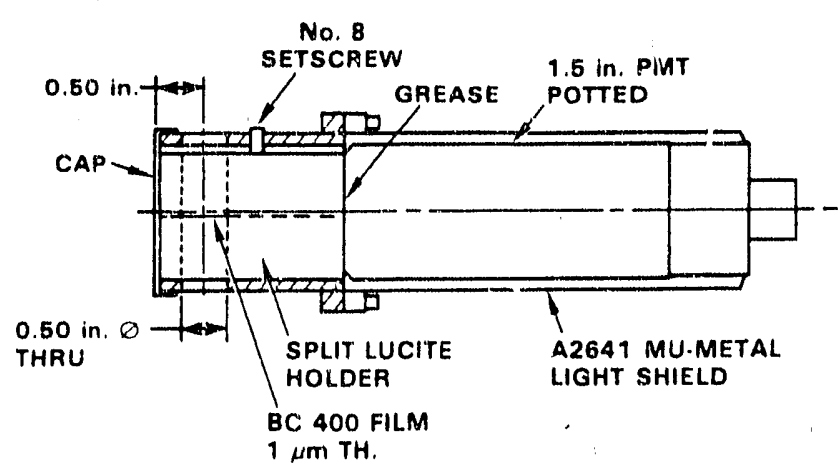

Figure 24. Light-pipe assembly details

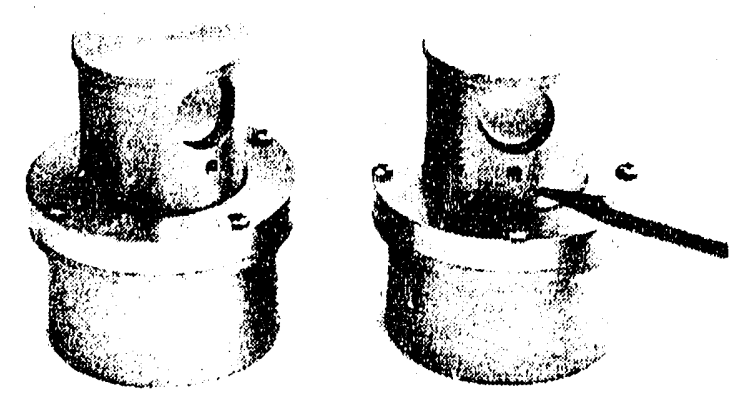

Figure 25. Setscrew used to mount the light-pipe assembly inside the aluminum holder

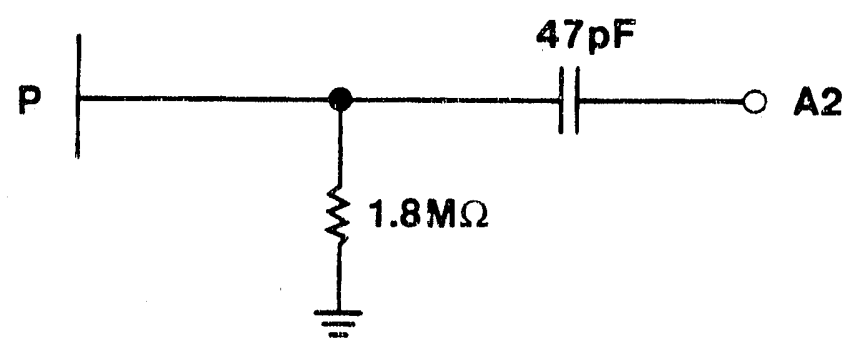

Figure 26. AC coupling used on the photomultiplier tube $\mathrm{A} 2$ output

\subsection{NIM Electronics}

The NIM electronic modules used for data acquisition are manufactured by EG\&G Ortec (Figure 27). Any specific information regarding these modules will be contained in the owner's manuals supplied by EG\&G Ortec.

\subsection{Dual-Channel Pulse-Height ADC with IE A PC DMA}

The following is a description of the electrical system used for measuring 2-parameter signals (TOF and PMT pulse height). The electrical system identifies the $\mathrm{Cf}-252$ fission fragments and correlates each fragment with single events in the DUT. The dualchannel pulse-height ADC is housed in an NIM unit (Figure 28). In addition to the circuits described below, the system employs an IBM PC-compatible personal computer with a parallel-direct memoryaccess interface (Metrabyte PDMA-16). The dualchannel ADC performs the same functions as commercially available, dual-parameter multichannel pulse-heigh t analyzers, but at a greatly reduced cost. This is bectuse large amounts of special memory and complicated hardware are used in the commercially available equipment to display data in real time. In addition, the dual ADC system provides the control logic necessary to correlate the 2-parameter measurements with single events, a feature that is not included in other commercially available equipment.

Figure 29 is a block diagram of the circuit in Appendix $B$ that detects the slope reversals on the time-of-flight (TOF/NIM1) and PMT pulse-height (LET/NIM2) analog-input signals and holds the peak value for 16-bit digitization. The timing diagram is shown in Figure 30. An increasing analog-input signal voltage charges a 1000-pF capacitor and forces the HOS 200 high-speed buffer-amplifier and the AD9686 comparator into the positive state. A subsequent slope reversal of the anslog input causes the 1000-pF capacitor to discharge, changing the state of the HOS 200 and the AD9686 to negative. This places the HTC-300A track-and-hold amplifier into the "hold" state, capturing the (inverted) peak value of the analog input. Lower-level discriminators (LLD1 and LLD2) allow for establishing minimum-peak detection voltages (typically 0.25 to $0.5 \mathrm{~V}$ ). Below the reference voltage, the $\mathrm{HTC}-0300 \mathrm{~A}$ remains in the "track" mode. The reset function is used to bring the HTC-300A back into the "track" mode after a peak detection has occurred. This circuit works with input pulses as fast as $250 \mathrm{~ns}$ FWHM.

Inverting amplifiers are used to convert the peak values into a positive voltage, and the AD1376 16-bit ADCs are used to digitize the result. The conversion time is $<17 \mu \mathrm{s}$. External TTL logic is used to provide an interface to the personal computer via parallel direct-memory access (PDMA-16 circuit board). 


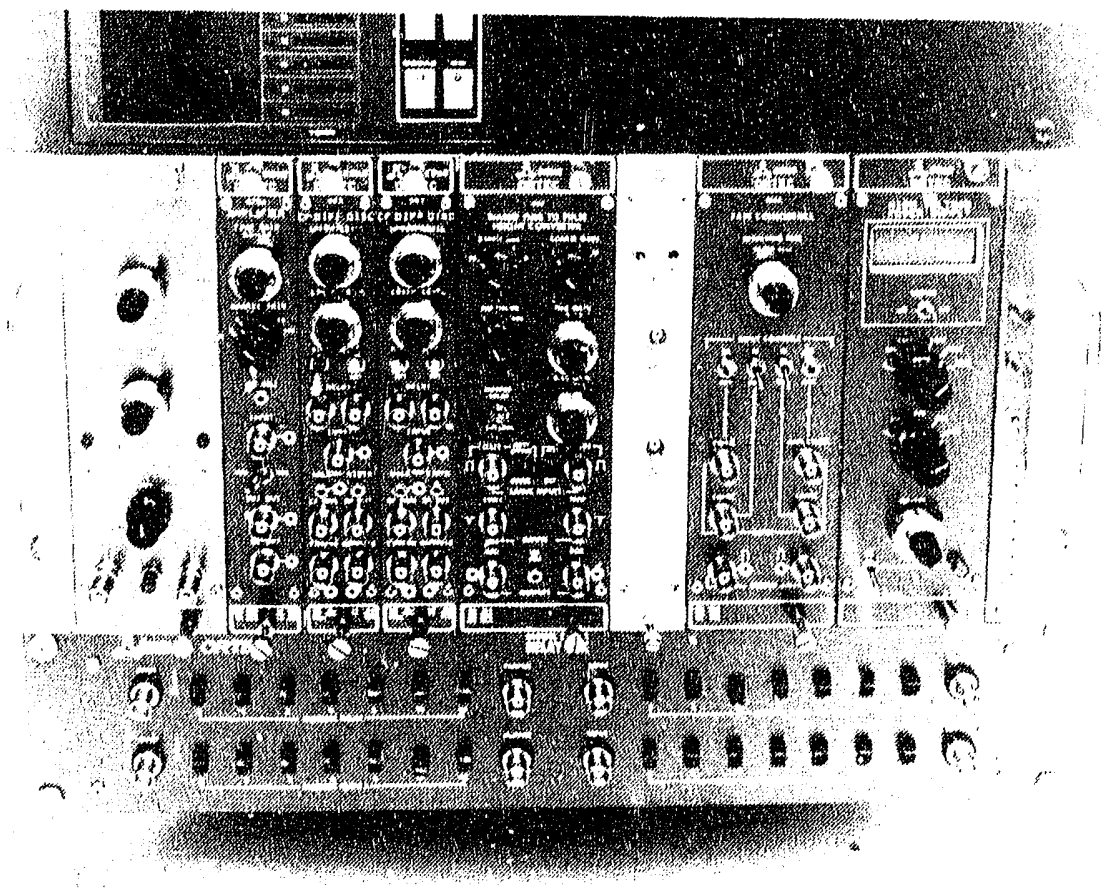

Figure 27. NIM electronics used for data acquisition. Left to right: 428 detector bias supply, 575A amplifier, two 583 constant fraction discriminators, 457 time-to-amplitude converter, dual-channel pulse-height ADC, 414A fast coincidence (optional), and the 556 high-voltage supply. Mounted below the NIM bin is the DB 46.3 delay.

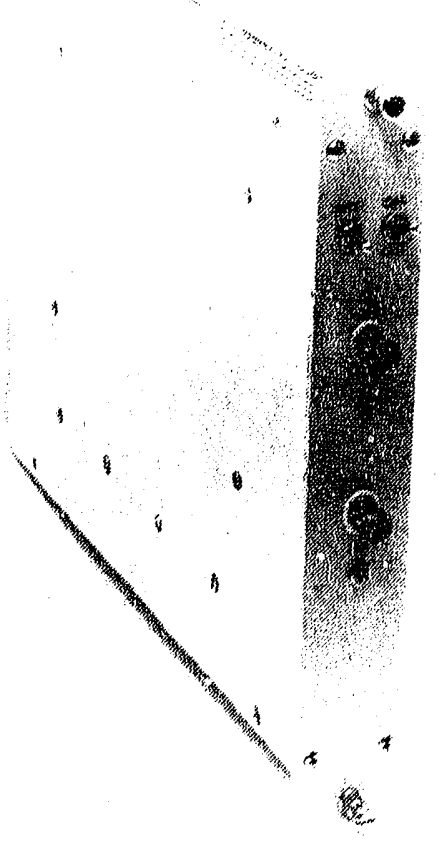

a. Front panel with reset and calibration/test switches, and the 'TOF' and LET' BNC' inputs

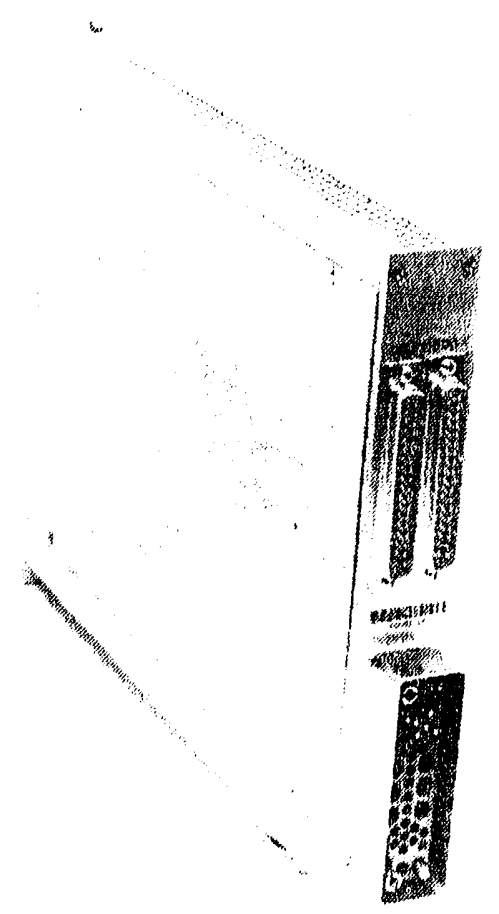

b. Rear panel with the I)MA and FI)( D)37 (male) connectors.

Figure 28. Iual-channel pulse-height. AI)C. 


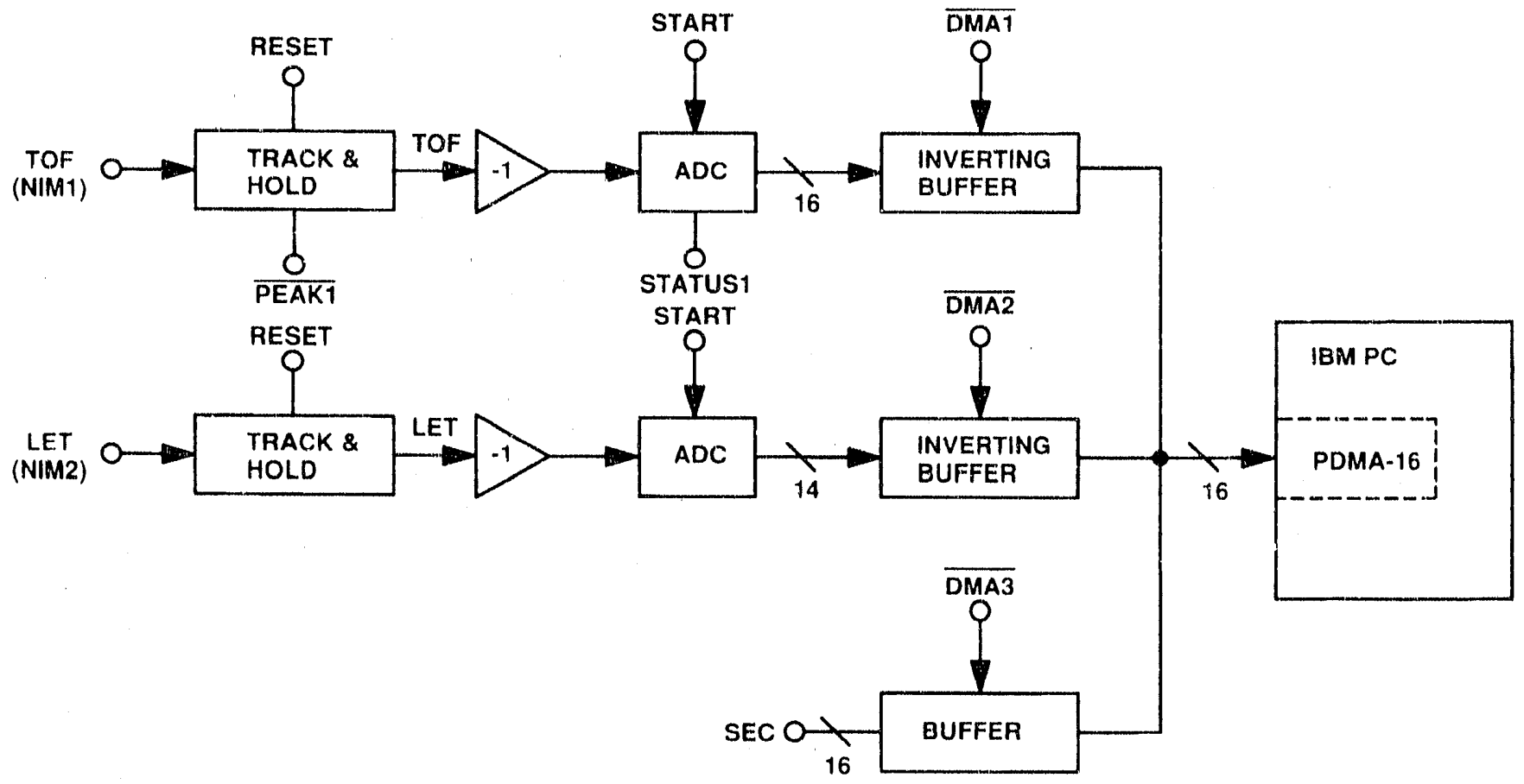

Figure 29. Functional block diagram of the dual-channel pulse-height ADC, 


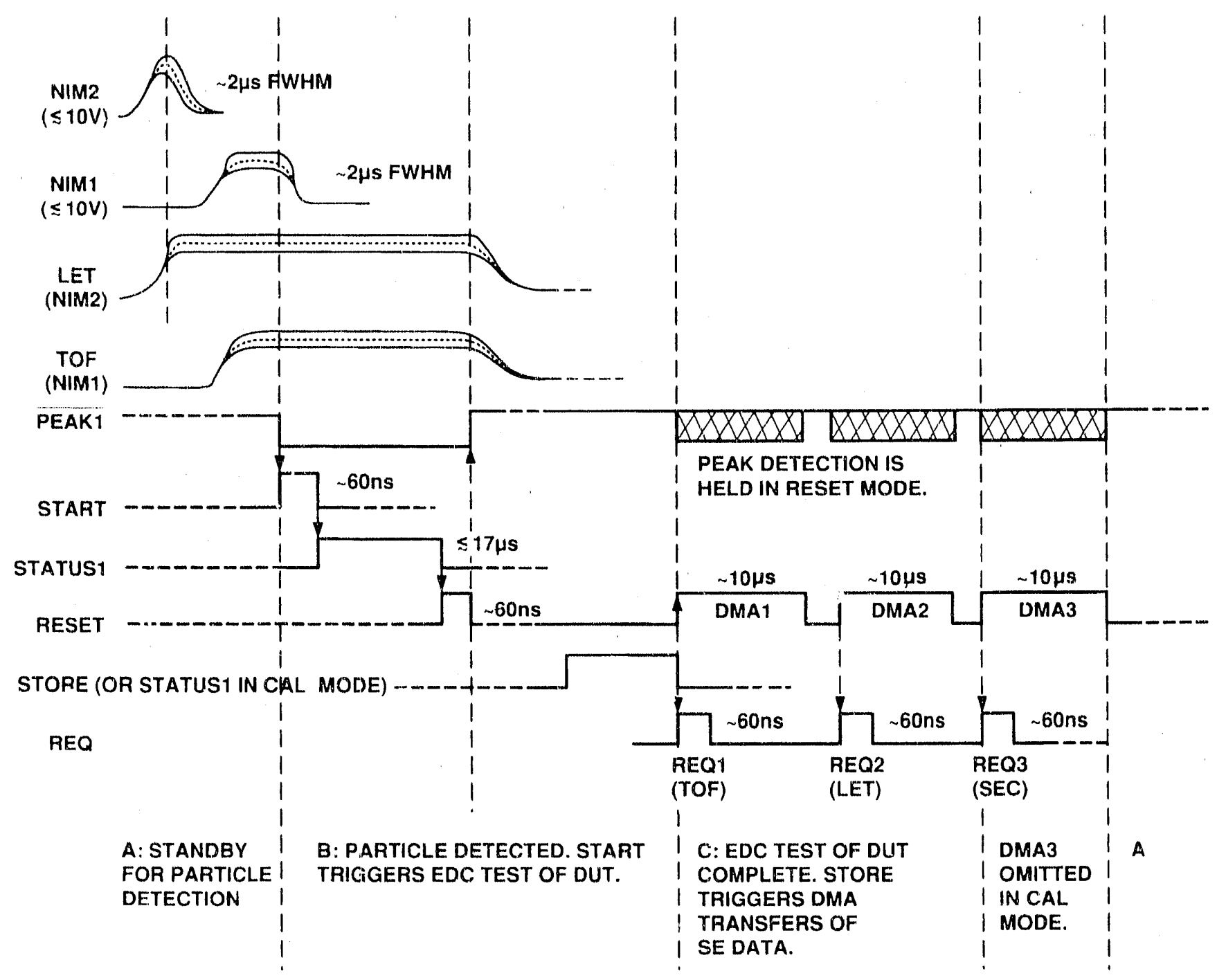

Figure 30. T'iming diagram for the dual-chamel pulse-height ADC. 
Upon receipt of a low state of /PEAK1, which indicates a peak value of the analog input has been captured, the control logic provides a START pulse to the AD1276 AI)C. The rising edge of /S'TATUS, which indicates that the digitization is complete, is used to reset the peak-detection circuit. The T"IL interface transfers data from the AD1376 ADCs to the personal computer upon receiving a control signal (STORE) from the user's test circuit. 'This accomplishes the correlation between single events and the fission fragments that cause them. The type of single event coded as 16 bits (or no single event detected, hexadecimal FFFF) is also transferred from the user's test circuit to the IBM PC. The data transfer is initiated by the REQ pulse's. Approximately $30 \mu \mathrm{s}$ is allocated for the transfer of the TOF and PM'T pulse-height data, and the single-event code (SEC).

\subsection{Software}

The man-machine interface for the Model 5005 - TF Single-Event Test Fixture is accomplished by means of software, written in the BASIC language, for an IBM PC-compatible personal computer. The software permits calibration of the system, provides control over single-event tests of the DU'T, and organizes the test data into a database for the user. A menu reference for the Model 5005-TF software is given in Figure 31.

The main menu (MODEL 50)5-TF SINGLEEVENT 'TEST' SYSTEM) presents the user with the options to go to other menus that permit

- calibrating the system

- defining the active buried layer and covering passive layers of the semiconductor DU'T'

- setting the parameters for a single-event test of the DUT

- beginning and ending a radiation exposure (single-event test.) of the DU'T

- displaying or printing a report of the data from a previous test

- manipulating data files.

The CALIBRATE SYSTEM menu lets the user specify the energies of the average heavy- and lightfission fragments for the current system configuration (('f-252 source and scintillation forils). The energies are measured using a solid-state detector placed at the DU'T position. The detector is first calibrated using a small, unencapsulated (C) 252 source to locate the channels of the unatenuated heavy and lightpeaks. Then the heavy- and light-peak channels are determined for the fission fragments as they exit the large source, after passing through the first and second scintillators. The channel values are used to calibrate the TOF and PM'T pulse heights for all of the fission fragments. The user must also specify the collimator area and the distance between the scintillators. Upon exiting the menu, the user may initiate a radiation exposure to complete the calibration of the system.

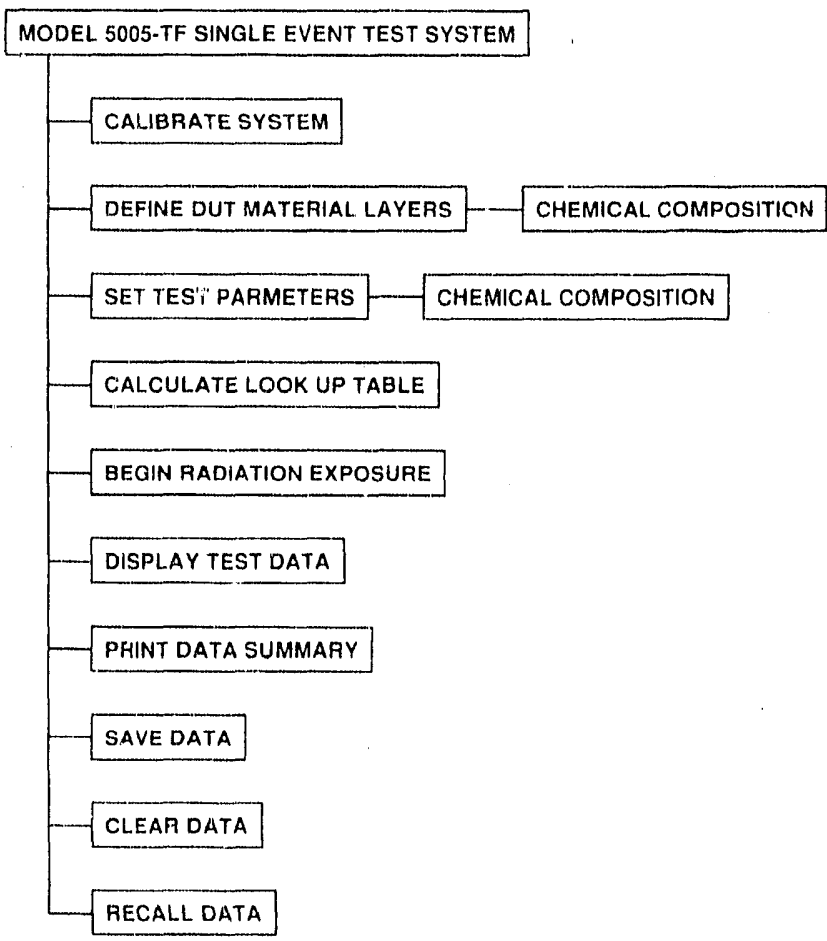

Figure 31. Menu reference for the Model 5005-TF software

The DEFINE DUT LAYERS and CHEMICAL. COMPOSITION menus allow the user to specify the thickness, density, and chemical composition of the buried active layer and the covering passive layers of the 1)(IT'. 'This information is used to calculate the energy loss of the fission fragments as they pass through the covering layers. It is also used to calculate the surface LET, average effective LET', and the exit LET of fission fragments in the buried active layer. Up to ten layers may be defined. The last laver specified is the active layer of the DU'T in which single events are assi!ned to occur. For each layer up to ten chemical elements, making up the layer may be specified in terms of atoms per molecule. If the material is a mixture and not a true chemical compound, then the percent by weight of each element in the mixture must be converted to a raiio. 'This ratio would be equivalent io the number of atoms per molecule had the mixture actually been a compound. 
The SET TEST PARAMETERS menu lets the user enter the test parameters for each radiation exposure of the DUT. These parameters are the test identification and the angle of incidence of the fission fragments on the DUT. Also included in the test parameters are the properties of the foril used to degrade the energy of the fission fragments after they exit the second scintillator and before they strike the DU'T. Upon exiting the menu, the user is asked to specify the chemical composition of the degrader foil.

When the system is calibrated and the material layers of the DU'T are defined, then a look-up table that associates the LET values with the estimated TOF and P".'T pulse height for all possible fission fragments is calculated. The table is used during the radiation exposure to display the single-event data as the data accumulate. After the look-up table has heen calculated, the user may begin the single event test of the DU'T by selecting that option in the main menu.

The remainder of the menus allow the user to view plot: or print a data summary of a previous single-event test (DISPLAY TEST' DA'TA and PRINT DATA SUMMARY' or to manipulate data files (SAVE DATA, CLEAR DATA and RECALL DATA). The user may save or recall data files containing the system calibration data, the DUT specifications, or the single-event test data.

\section{Operation}

\subsection{Theory of Operation}

The Model 5005-'TF' Single Event 'Test Fixture incorporates a TOF measurement ${ }^{2}$ and an ultra-thin scintillation foil-pulse $h$ ht measurement:" 11 to identify the (1.25) fission-iragments, and correlates the particle information with single events that are detected in the DI'T. When ( $7-252$ spontaneously fissions. it produces two fragments with the average characteristics" listed in 'able?.

\section{Table 2. Average Heavy- and Light-Fragment Characteristics}

\begin{tabular}{lcc} 
& Heavy & Light \\
\hline A & 142.1 & 10.5 .9 \\
$Z$ & 55.6 & 42.5 \\
T(MeV & 79.5 & $1(1.4 .1$ \\
\hline
\end{tabular}

Conservation laws of physics require simultaneous satisfaction of the following equations.

Mass: A-heavy + A-light + neutrons $=252$

Energy: T-heavy + T-light $=82.5 \mathrm{c}$ $\left( \pm 2.5^{\prime \prime}\right)$ energy of fission

Momentum: (A'T)-heavy $\cong\left(A^{\prime} T\right)$-light

The energy released by the fission reaction is calculated from the nuclear-binding energy approximation. $12,1: 3$

BE $(\mathrm{MeV} / \mathrm{amu})=9.24-0.00696 \mathrm{~A}(\mathrm{~A}>77)$

A fission fragment's TOF is related to the particle's energy and mass. In general, light fragments have higher energies and thus shorter 'TOF than the heavy fragments. Figure 32 shows the uncertainty in the fission-fragment mass given only a TOF over 10 (1). The maximum error in the surface LET' in silicon is about $\pm 10 \mathrm{MeV} \mathrm{cm} / 2 / \mathrm{mg}$ for this one-parameter method of particle identification.

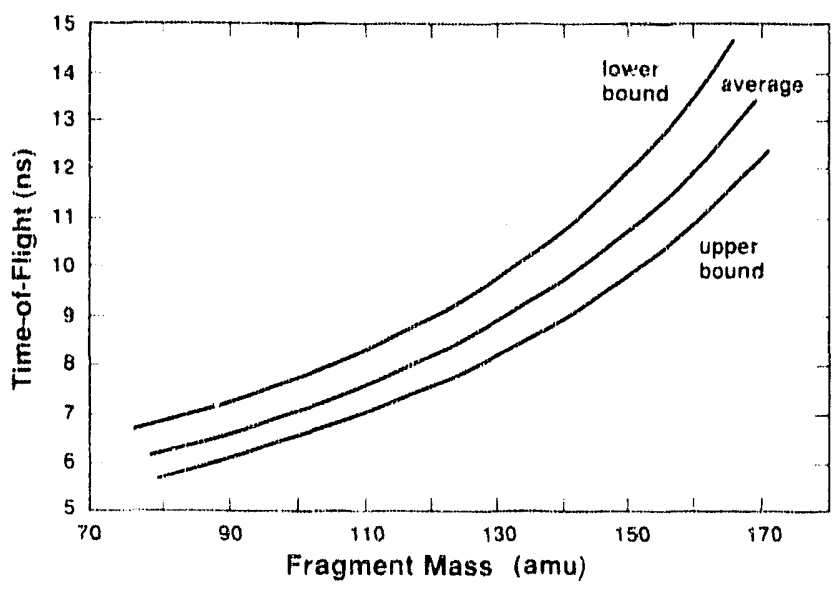

Figure 32. Uncertainty in the fission fragment mass as a function of the time of flight over $10 \mathrm{~cm}$

The precision of the surface LET measurement. can be improved to about $\pm 1 \mathrm{MeV} \mathrm{cm}^{2} / \mathrm{mg}$ by using the light output from one of the ult ra-thin scintillators in coincidence with the TOF. The PMT output is empirically related to the energy loss of the fragment. to the scintillator." The tube output thus provides a measurement of the fragment's energy loss (change in velocity) in the scintillation plastic. (The energy loss 
in the second TFD is uniquely given by the combination of the TOF between the two scintillators, and the average velocity in the second scintillator film. The average velocity is proportional to the light output from the scintillator film. ${ }^{2}$ ' 'Thus ${ }^{1.4}$

Z-strip" $=\frac{\text { LET'ff }}{\text { LET-proton }}$ Z-proton ${ }^{2}$

where the proton's velocity is the same as the fission fragment's velocity, $V$, which is determined from the TOF, then all of the quantities in Eq (5) are known. 'The strip charge of the fission fragment is also given by ${ }^{1: 1}$

$$
\begin{aligned}
Z \text {-strip }= & Z\{1-[1.039-(0.17777 \exp ) \\
& (-(0.08114 Z)] \exp (-\mathrm{V} 2)\}
\end{aligned}
$$

where

$$
\begin{aligned}
& V_{2}=V_{0}+0.378 \sin ^{2}\left(V_{1} \pi / 2\right) \\
& V_{1}=0.886(\mathrm{~V} / \mathrm{V} 0) \mathrm{Z}^{2 / 3}, \text { and } \\
& V_{0}=\text { the Bohr velocity. }
\end{aligned}
$$

'The only known quantity in $\mathrm{Fq}(6)$ is the atomic number of the fission fragment, $Z$, which can be solved for by substitution of the strip charge from Eq (5). 'The mass of the fragment, $A$, can be estimated from the most probable charge of the fission reaction. ${ }^{16}$

$$
\begin{aligned}
& \text { A-heavy group }=2.46 Z-3.14 \\
& \text { A-light group }=2.43 \mathrm{Z}-3.35
\end{aligned}
$$

The energy of the fission fragment is estimated from the measured particle velocity, $V$, and the estimated mass, A. 'Thus all of the particle parameters (charge, mass, and energy) can be estimated from TOF and energy-loss measurements.

\subsection{System Pumpolown and Venting}

Assuming that the entire system has been assembled, the following is a step-by-step procedure for both pumping the svistem down and venting to the atmosphere.

\section{Pumpdown.}

1. Close the source-access shutter.

2. Close the vent value on the back of the (hamber (Figure 21).
3. Close the chamber door and latch it shut (Figure 333).

4. Turn on the roughing pump.

Note: A loud gurgling sound will be present during the initial few seconcis. As the roughing vacuum increases, this noise should diminish. If after 10 to $1 \mathrm{i}$ sec you do not hear a substantial reduction in noise, this could mean the system has a major leak. If this occurs, shut off the pump and investigate for possible leaks.

5. Turn on the turbo pump (Figure 34).

Note: 'The convection gauge on the (iP controller reads the roughing vacuum pressure and is always on (Figure 35). To read the high-vacuum pressure the ion gauge is used. Do not turn it on until the convection pres. sure reads $<5 \mathrm{E}-3$ torr. The system is ready for use when the vacuum reaches $<2 \mathrm{~F}-4$ torr.

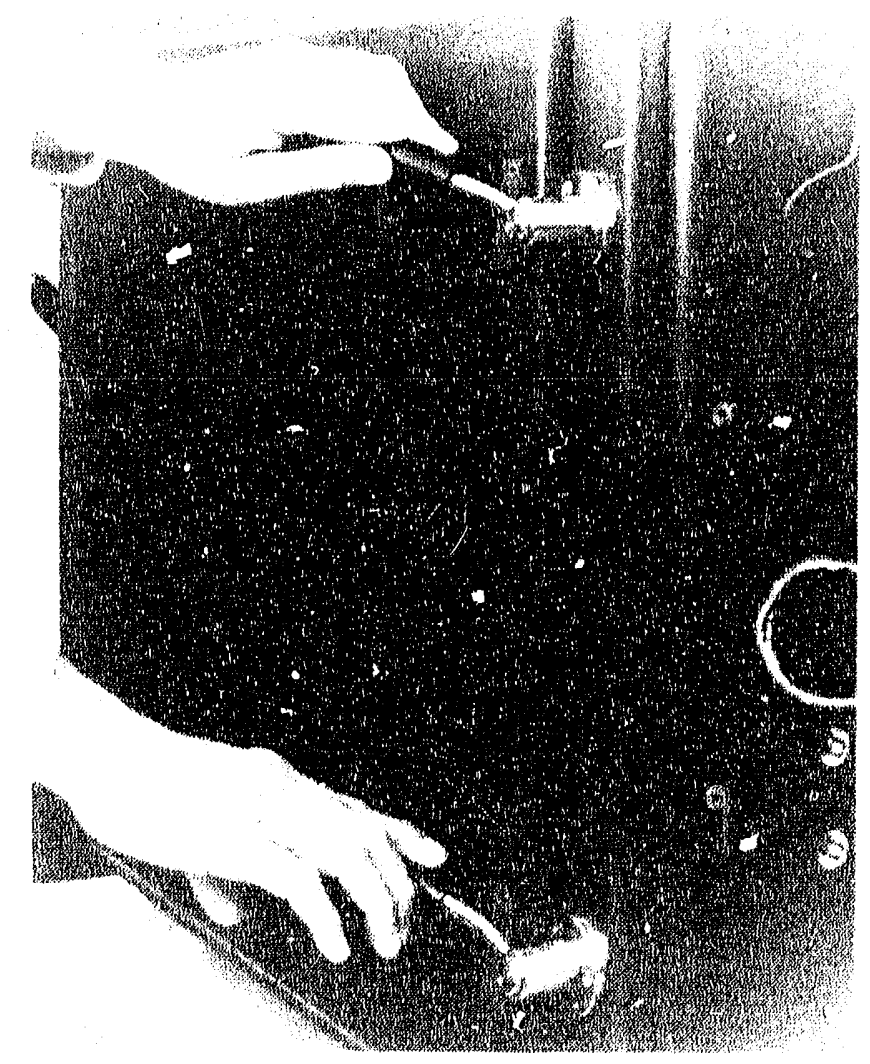

Figure 33. Vacuum chamber door in the latched position 


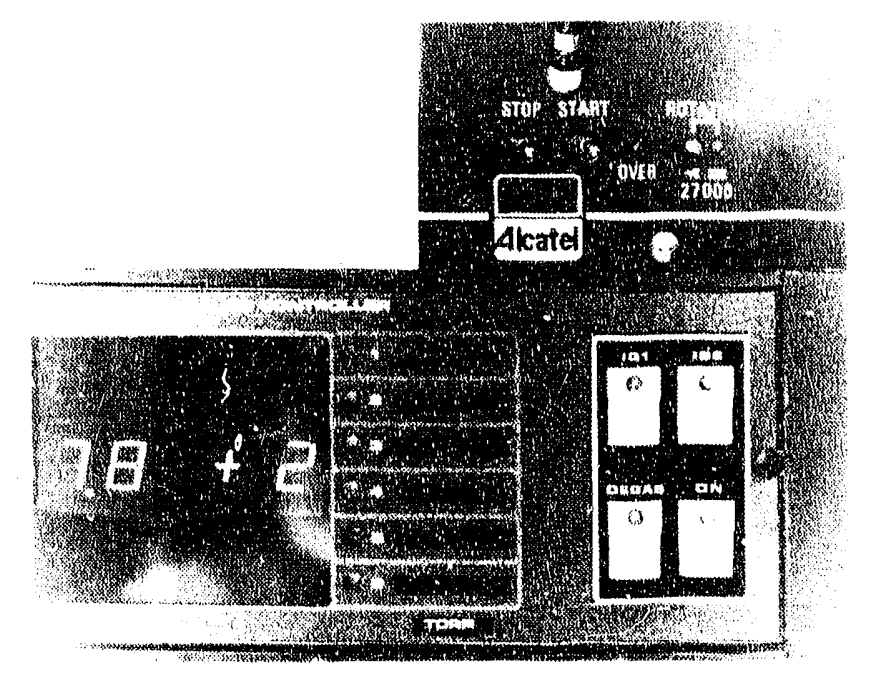

Figure 34. Turbo-pump switches

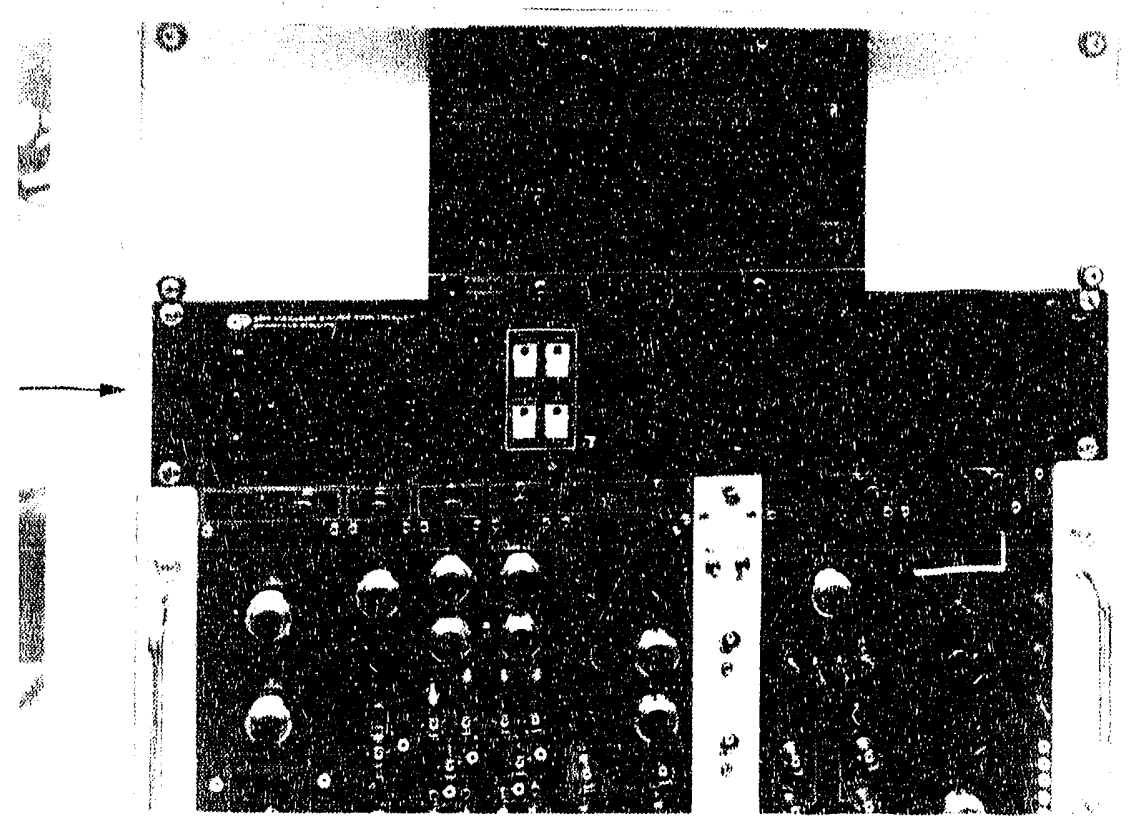

Figure 35. Vacuum-pressure indicators and switches

\section{Venting}

1. 'Turn off' the high voltage to the PMT's (Figure 36).

2. Turn off the ion tube (Figure 37).

3. Turn off the turbo pump.

4. Turn off the roughing pump.
5. Open the vent valve on the back of the chamber.

Note: 'To keep the inside of the chamber free from dust and moisture, plumbing a drynitrogen gas line to the vent valve is recommended.

6. Unlatch the door of the chamber. When the door opens slightly, the system is vented (Figure 38).

7. Close the vent valve. 


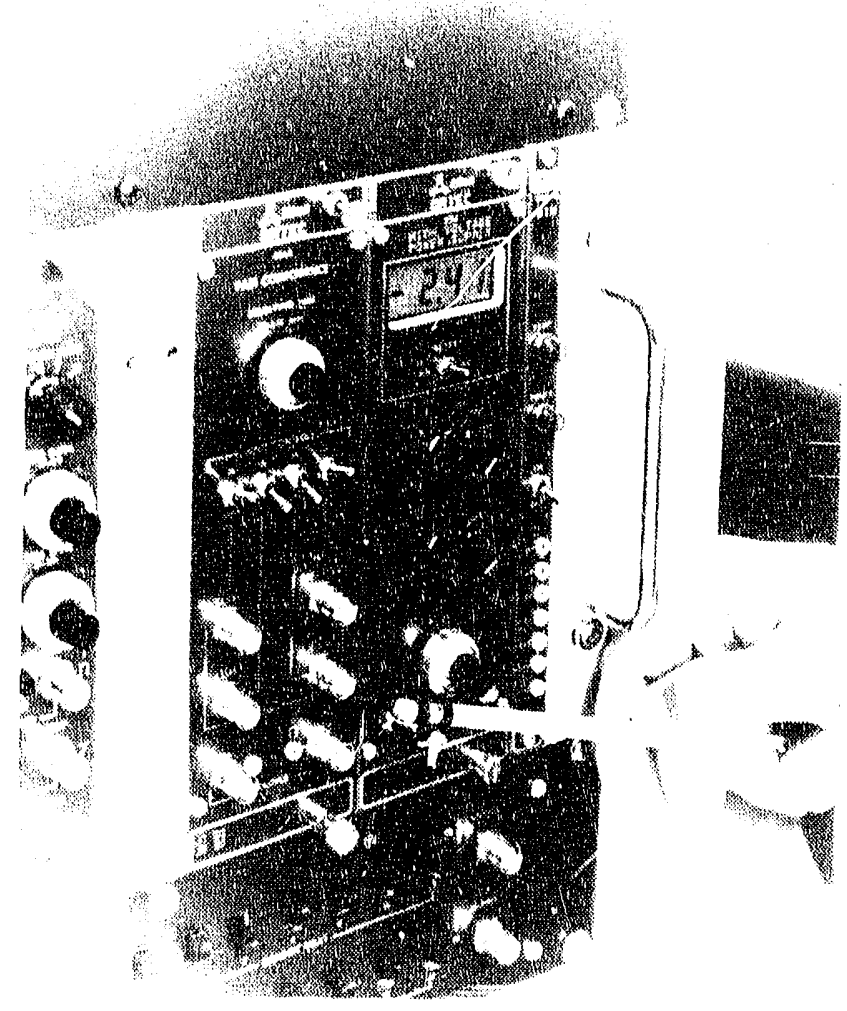

Figure 36. High-voltage switch

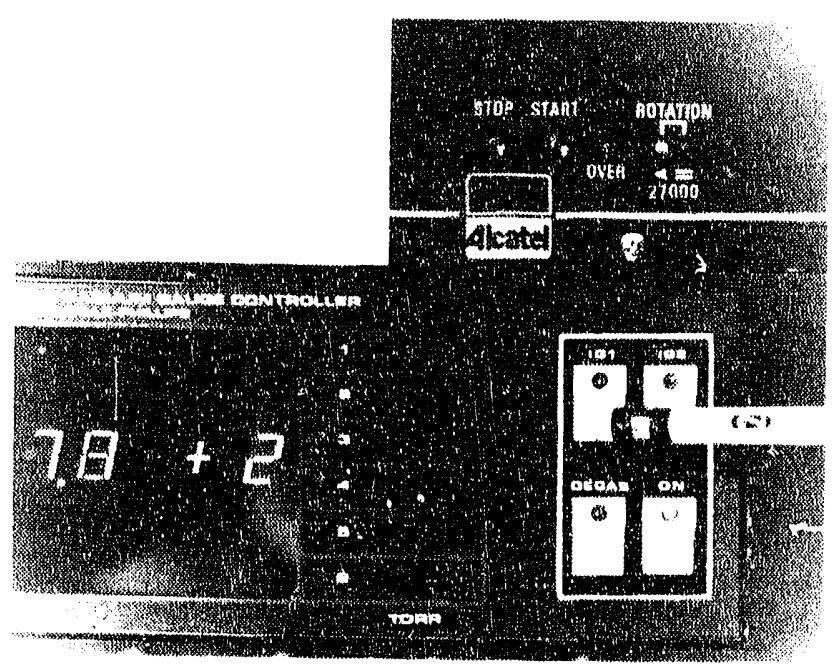

Figure 37. Ion-gauge switch 


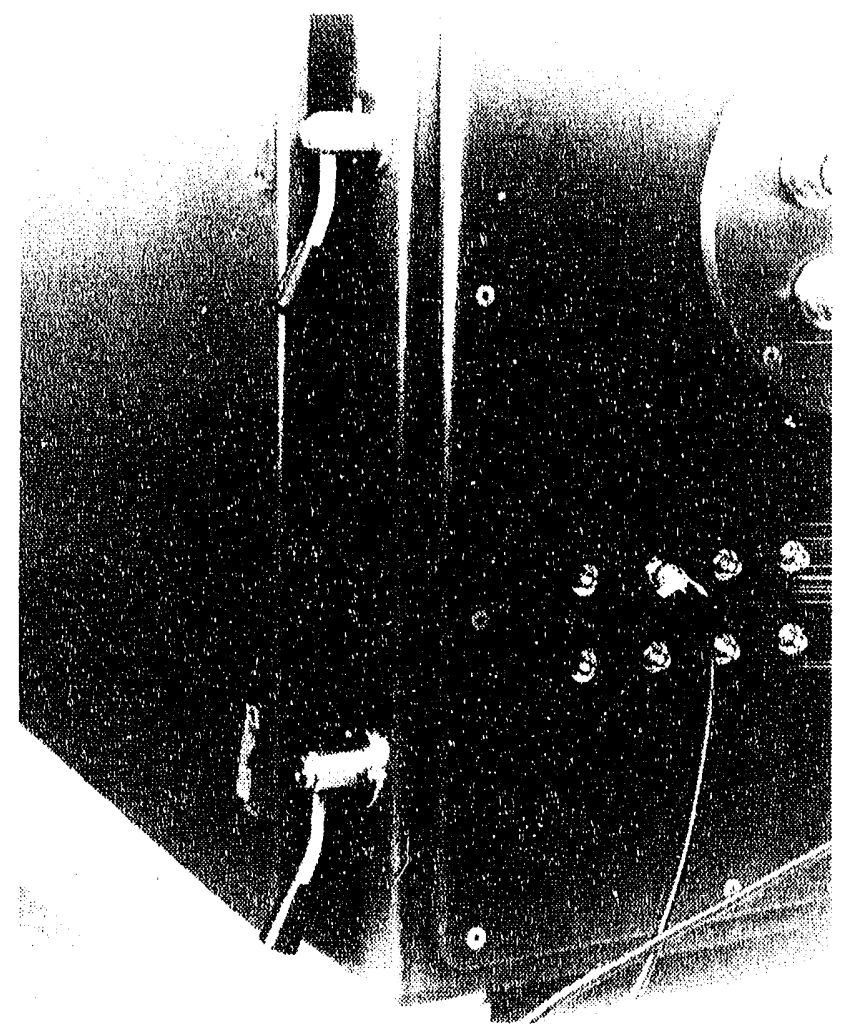

Figure 38. Vacuum chamber door in the unlatched and vented position

\subsection{System Calibration}

Before the Model 5005-TF can be used for SEU testing, the system must be ca'ibrated. 'The objective of the following procedure is to obtain the data necessary to convert the TOF and PM'T voltage-pulse heights into particle mass and energy estimates. The procedure consists of two parts: fission-fragment energy-spectra calibration, ${ }^{17}$ and ${ }^{\text {TOF }}$ and LET pulse-height calibration. The equipment provided with the Model 5005-TF will be required for the procedure. In addition, a 512 -channel, or better, multichannel analyzer (10-V input range) and a 100 )$\mathrm{MHz}$ digital-storage oscilloscope will also be required.

\section{Energy Calibration.}

1. Set up the NPI), with the calibration source shown in Figure 39a, following the wiring diagram in Figure 40.

2. Close the vacuum chamber and pump down the systern.

3. Adjust the $575 \mathrm{~A}$ amplifier or the MCA gain until you can record a fission-fragment energy spectrum similar to Figure 41.

4. Record the channel numbers corresponding, to $\mathrm{PL}$ (right peak maximum) and $\mathrm{PH}$ (left peak maximum) in Figure 41.

5. Repeat the procedure above for the large test source. 'The channel numbers for PL and PH should be slightly less than the PL and PH values for the calibration source. 'The difference is a result of the energy of the fission fragments that is attenuated by the gold cover layer on the large source.

6. Install the first TFI) between the large test source and the NPD shown in Figure 39b.

7. Close and pump down the vacuum chamber.

8. Acquire the fission-fragment energy spectrum and note the values of $\mathrm{PL}$, and $\mathrm{PH}$.

9. Install the second TFD as shown in Figure $39 \mathrm{c}$, and repeat the procedure above.

Now, you should have four sets of PL and PH channel numbers describing the energy attenuation of the fission fragments as they pass through the gold layer of the large source and through the first and second TFDs. The energy calibration data permit the energy spectrum of the fission fragments incident on a DU'T to be calculated. This completes the first part of the calibration procedure. 


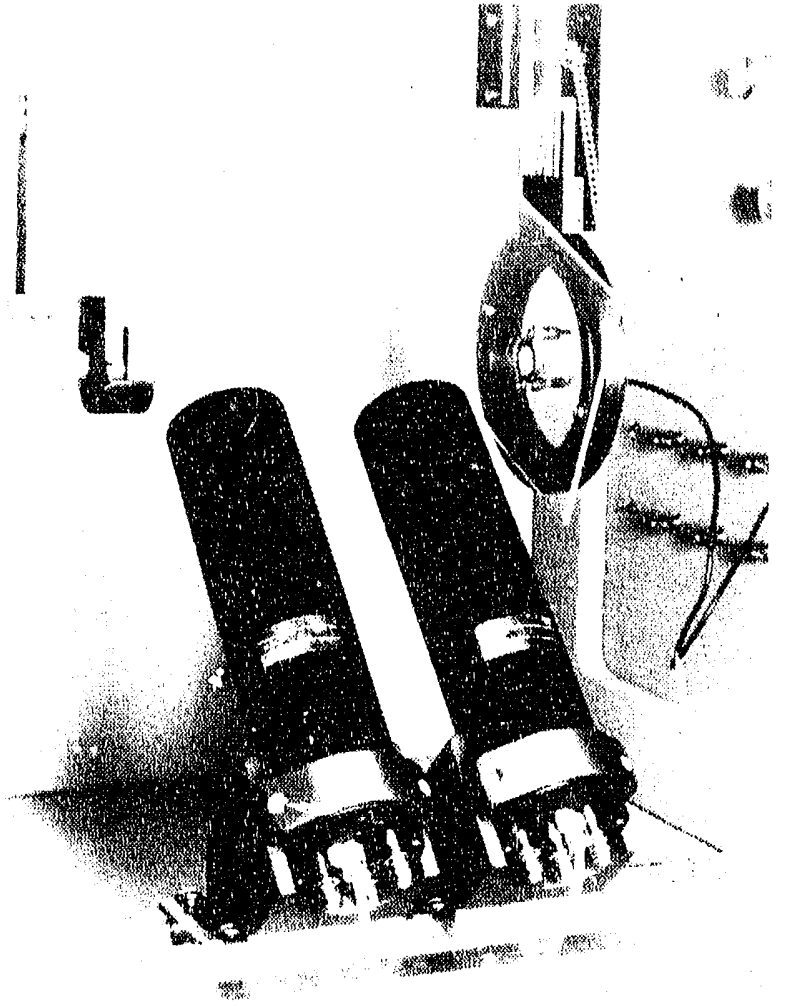

a. Calibration and test source installed

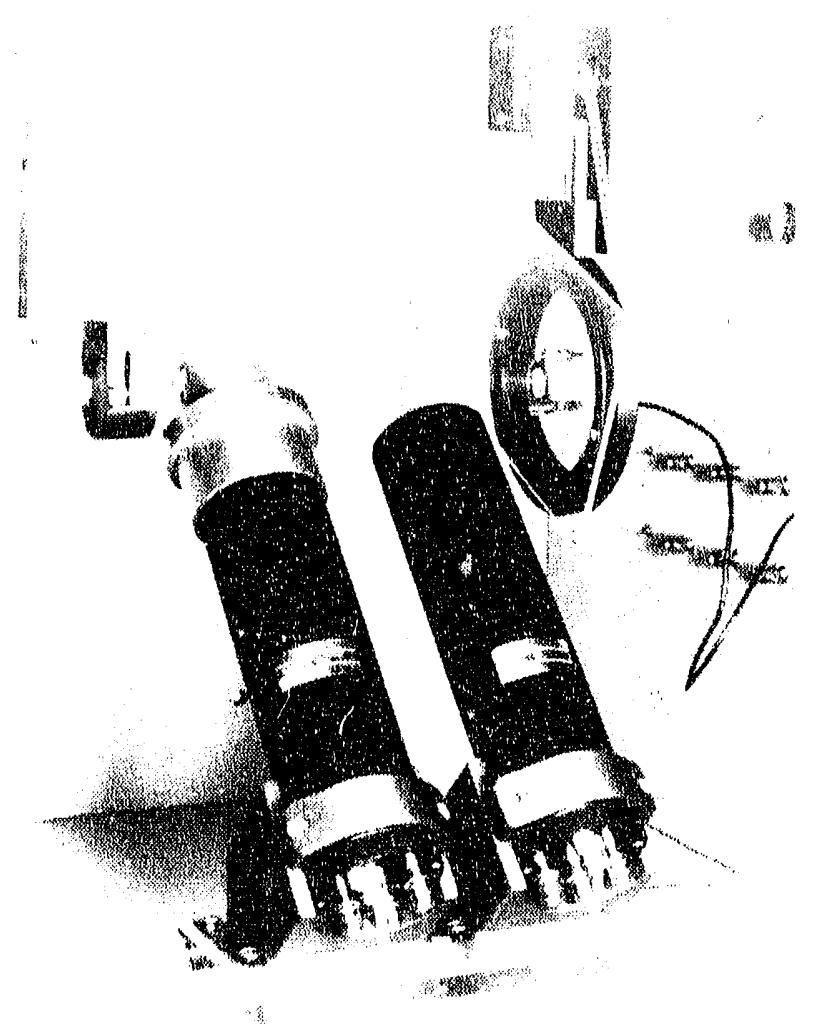

b. First T'FD installed

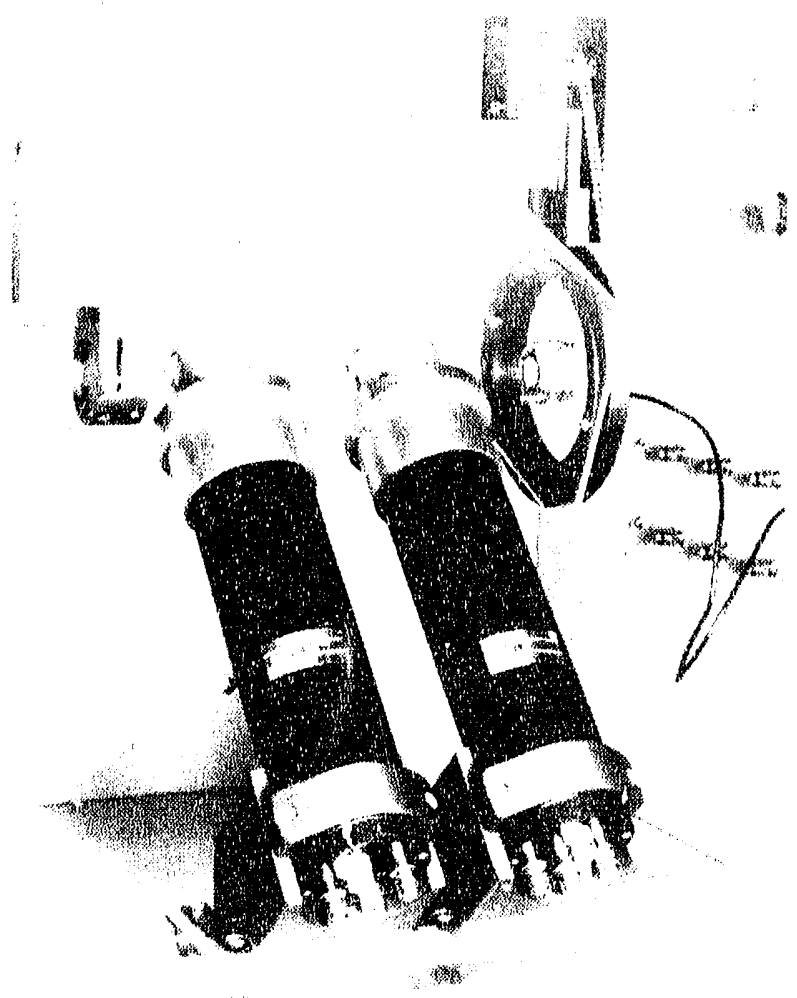

c. First and second TFlos installed.

Figure 39. Source and detector configurations for calibration of the Model bons-TF. 


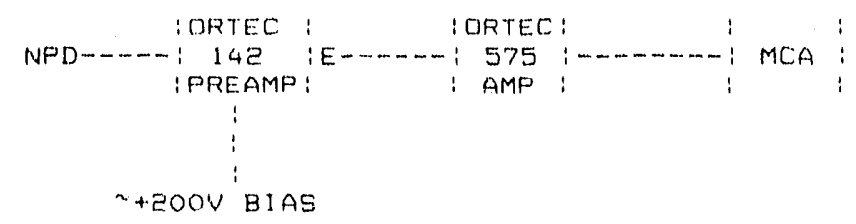

Figure 40. System wiring diagram for calibration of the Model 5005-TF

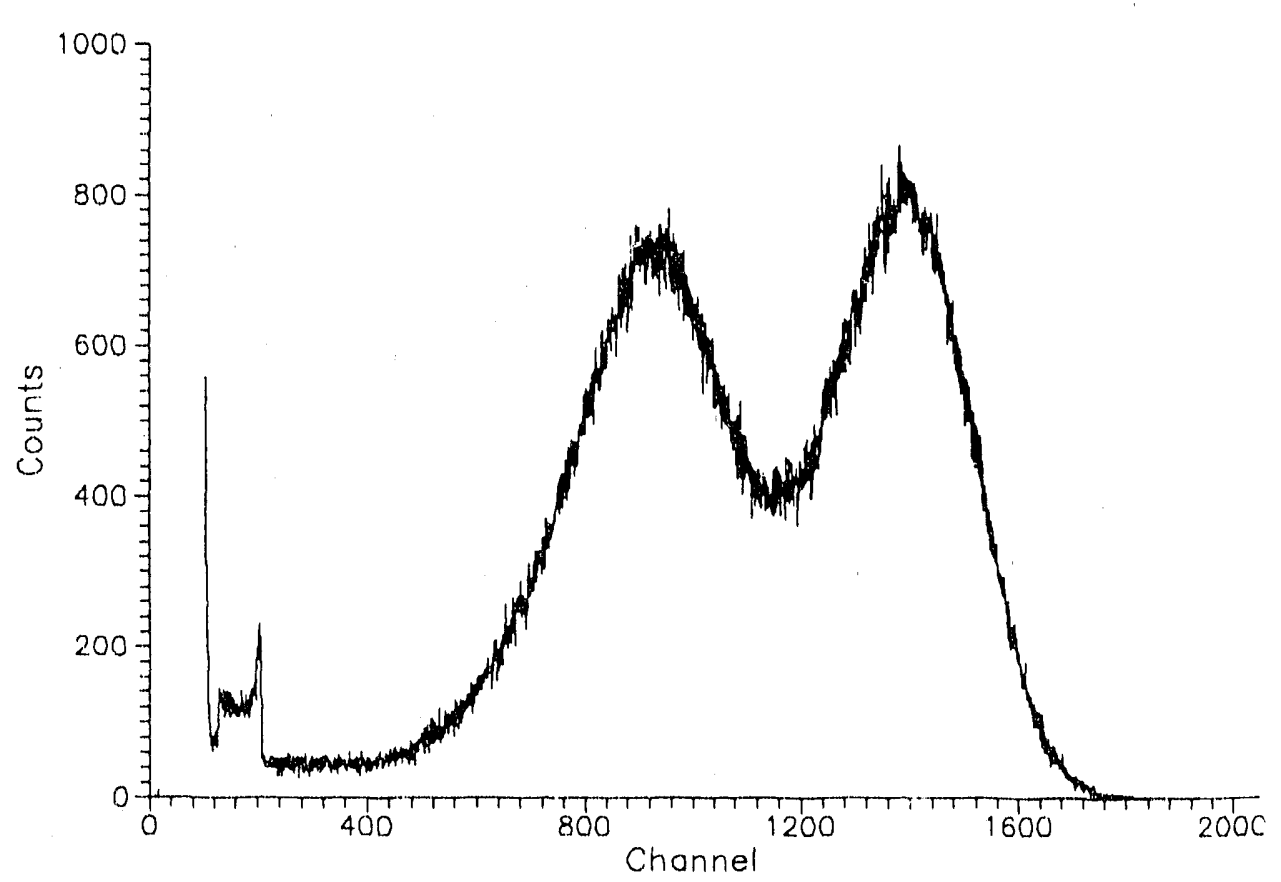

Figure 41. Cf-252 fission-fragment energy spectrum. Data obtained at Boeing Aerospace and Electronics Center, June 24, 1990.

\section{Pulse-Height Calibration.}

1. Set up the Model 5005-TF according to the system wiring diagram in Figure 10.

2. Select a separation distance between the scintillators for the TOF measurement.

3. Close the vacuum-chamber door and turn on the high voltage to the P'M'T's.

4. Bias the P'MT's to about -...2400 V.

5. Using an oscilloscope examine the output signals from the $58: 3$ discriminators.

6. Adjust the discriminator levels to eliminate signal noire caused by the PM'T dark currents.

7. Now turn off the high voltage to the PM'T's and pump down the system.
8. When the vacuum level is $2 \mathrm{~F}-4$ torr or less, turn off the ion gauge and turn on the high voltage to the PMT's.

9. Using the oscilloscope examine the ' $\mathrm{TOF}$ and LET signal inputs to the ADC unit.

10. Adjust the gains of the 457 time-to-amplifier converter and the $575 \mathrm{~A}$ amplifier until the pulse heights are between 1 and $9 \mathrm{~V}$. For each PM'T dynode pulse there should be a 'TOF pulse, and vice versa.

11. 'Trigger the uscilloseope in dual-channel mode from both signals and verify that the other signal is always presert.

12. 'Tum on and boot the IBM PC:

1.3. 'T'o access the Model 5005-T't soltware from the root directory, execute "(I) $\backslash 50055{ }^{\prime} \mathrm{TH}$." 
14. Execute "5005 $\mathrm{TF}$.

15. From the main menu select option "O-CALIBRATE SYSTEM" and enter the four sets of PL and PH channel numbers and the scintillator separation distance.

16. Press the space bar and answer " $y$ " to the prompt "CALIBRATE ADC." Answer "n" to the "REVIEW PREVIOUS CALIBRA'TION DATA" prompt.

17. Reset the ADC unit using the front panel "RST" switch.

A two-dimensional plot of the TOF and PMT voltage-pulse heights should begin to accumulate. (The display is updated after every 100 particle events.) After a few thousand events have accumulated, a fission-fragment spectrum similar to Figure 42 should be clearly discernible. The display is a two-dimensional plot of the base-10 logarithm counts for the voltage pairs. The total counts are represented by the single-digit exponent.

1. If the alpha-particle peak is present in the lower left-hand corner, then press the space bar (twice) to exit.

2. Incrementally raise the discriminator levels or reduce the $\mathrm{PM}^{\prime} \mathrm{T}$ bias voltage, and repeat the above procedure until the alpha peak is no longer present in the two-dimensional fission-fragment spectrum.

3. Press the space bar to exit.

'The voltage pairs for each of' the fission-fragment peaks are automatically calculated, and are denoted on the plot by asterisks. The logarithms of the total channel counts for the $\mathrm{TOF}$ pulses are displayed down the right of the plot, and across the top of the plot for the I.F'T pulses. The logarithm of the total number of particles detected is shown in the upper right-hand corner of the plot. Press the space bar to exit. 'The CALIBRATE SYSTEM menu should resemble Figure 43. Press the space har to exit and answer "n" to the "CALIBRA'TE AI)(" prompt. 'The Model 6005-TF' is now calibrated and ready for singleevent testing of microelectronic devices.

\subsection{Single-Event Testing}

The four preparatory steps for obtaining a singleevent characterization of a microelectronic device are construction of the event-detection circuit, DU'T definition, test parameter specification, and calculation of the look-up table. After the preparatory steps have been completed, the Model 5005-TF can be used to acquire single-event data. The IBM IC software permits review of the results of previous single-event. tests and errors in the calibration or test set-up steps to be corrected.

\section{Construction of the Event-Detection Circuit.}

The event-detection circuit is the interface between the Model 5005-TF and the DU'T supplied by the user. The circuit must be designed to perform five functions:

1. Initialize the DUT for a single-event test

2. Operate in a standby mode awaiting a signal from the Model 5005-TF (PFAK1 or /STAR'T) that a particle has been detected

3. Examine the IU'T for single events

4. Provide a signal to the Model 50(05-TF (STORE) to indicate that the single-event test has been completed and an SEC is to be recorded with the particle pulse-height data

5. Operate in a standby mode awaiting a signal from the Model 5005-TF (DMA3 or / REQ3) that the single-event data have been transferred to the $1 \mathrm{BM} P \mathrm{PC}$.

Figure 44 is a flowchart of the testing sequence. 'The event-detection circuit is cabled to the Model 5005-TF through the D,37 male connector labelled "EDC," and mounted on the rear panel of the dual-channel pulseheight AI)C unit. 

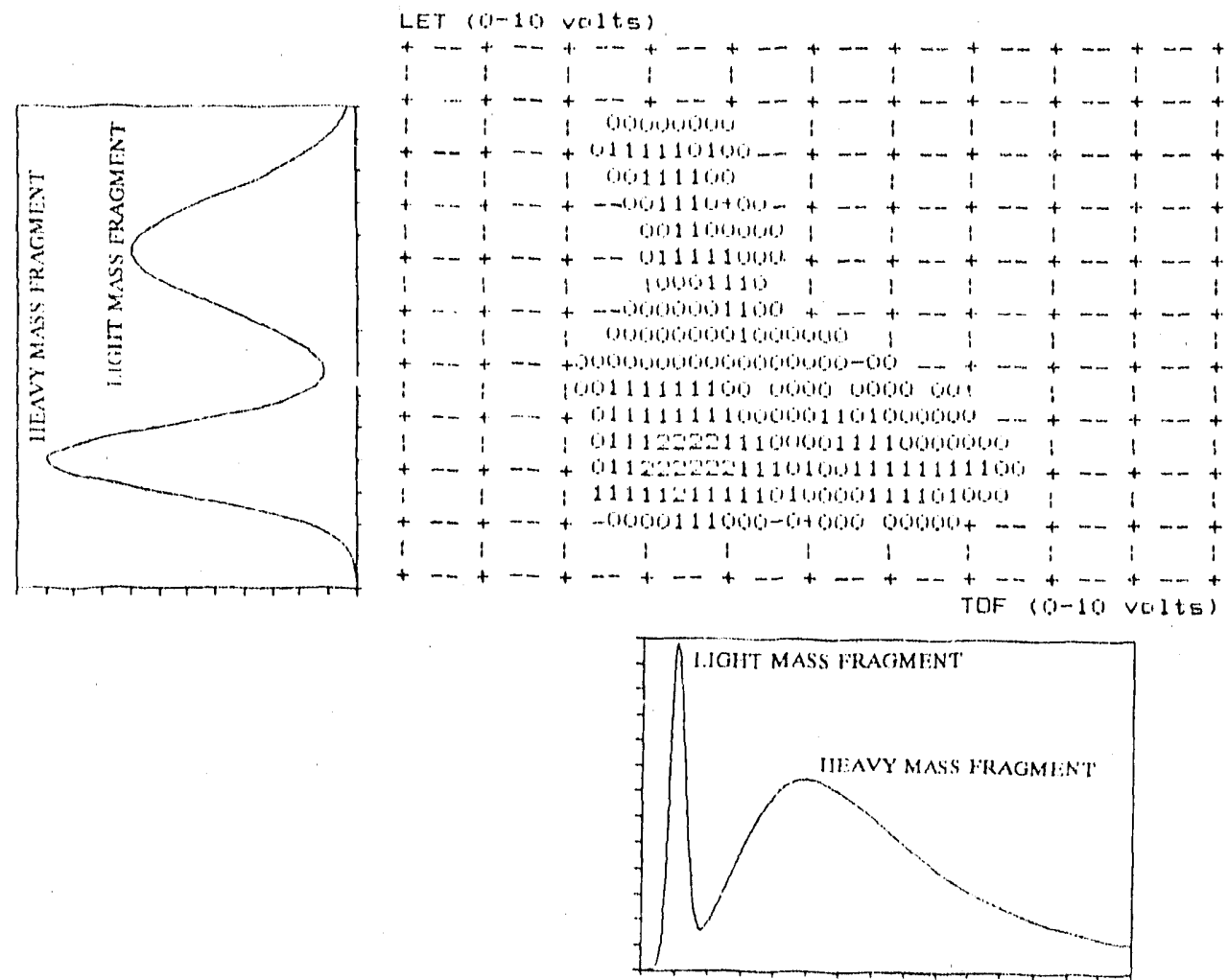

Figure 42. TOF vs. LFI pulse-height spectrum. Data taken at Lockheed Missiles and Space Company, April 4, 1990). Shown are the light-mass fragment group (upper left), the heavy-mass fragment group (lower right), and the alpha particles (lower left). 'l lhe digits are $\log _{10}$ counts for the voltage pair ('TOF and $L E T$ I ). The theoretical TOF and PMT pulse-height spectra are shown on the axes for comparison.

CALIBRA'TE SYSTEM (calibrated) new

$\begin{array}{lll}\text { TOF L-PEAK: } & 3.76 \text { volts } \\ \text { TOF H-PEAK: } & 6.09 \text { volts } \\ \text { LET L-PEAK: } & 6.44 \text { volts } \\ \text { LET H-PEAK: } & 2.32 \text { volts }\end{array}$

PRESS TO

SELECT

$\begin{array}{llll}0 & \text { CALIBRAIION SOURCE FL: } & 1128 & \\ 1 & \text { CALIBRATION SOURCE PH: } & 788 & \\ 2 & \text { LARGE SOURCE PL: } 1100 & & \\ 3 & \text { LARGE SOURCE PH: 776 } & \\ 4 & \text { PL AFTER FIRST SCINTILLATOR: } & 1012 \\ 5 & \text { PH AFTER FIRST SCINTILLATOR: } & 712 \\ 6 & \text { PL AFTER SECOND SCINTILLATOR: } & 972 \\ 7 & \text { PH AFTER SECOND SCINTILLATOR: } & 596 \\ 8 & \text { COLLIMATOR AREA: 1.27 (Sq.Cm) } \\ 9 & \text { SCINTILIATOR SEPARATION: 30 (cm) }\end{array}$

PRESS THE SPACE BAR TO EXIT

Figure 43. Example SYS'THM ('ALIBRA'TON menu 


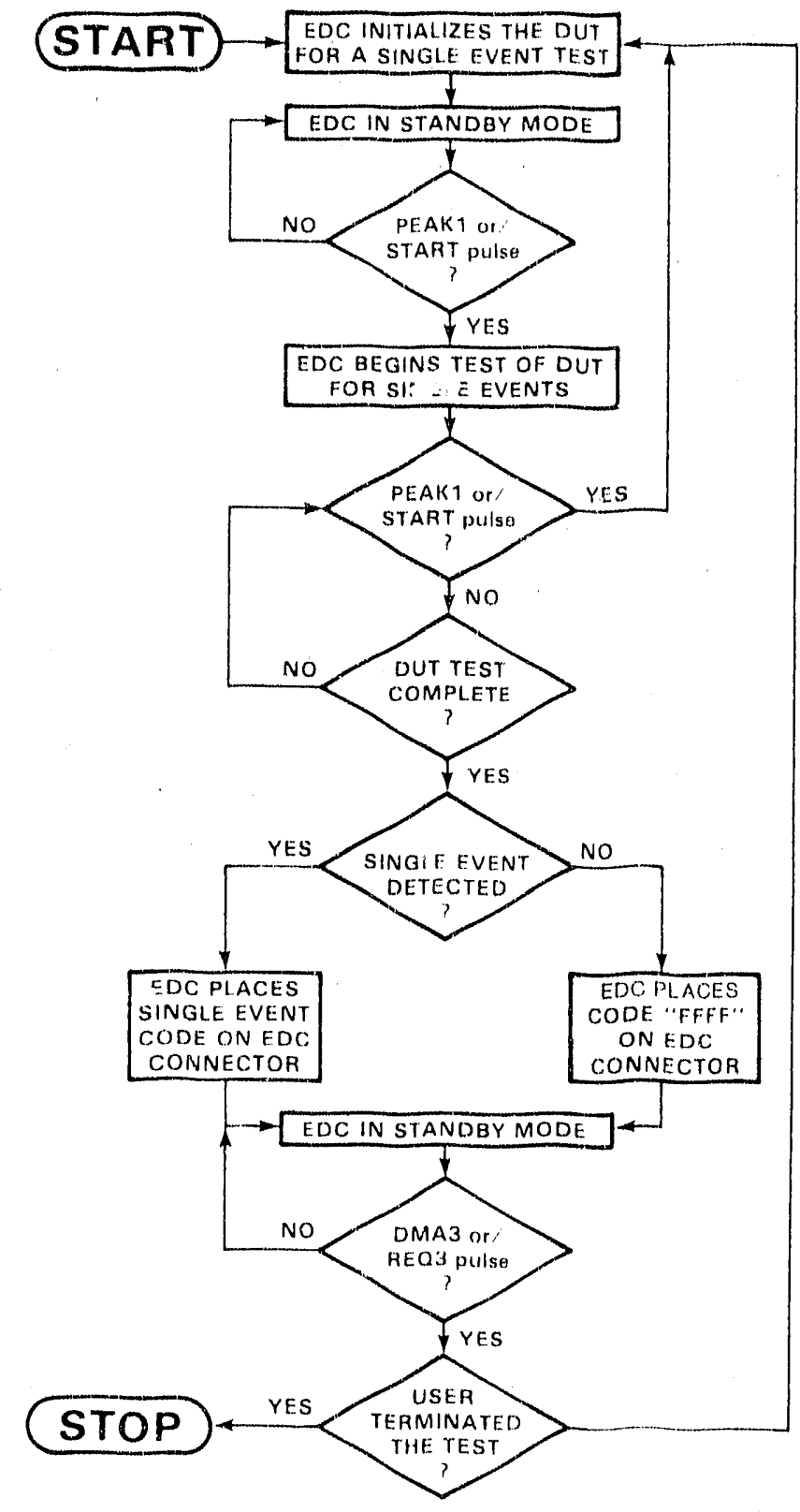

Figure 44. Flowchart of the single-event testing sequence
DUT Definition. 'The Model 50) - T'T IBM P': soltware will calculate the surface Latr, the averageeffective LL'T', and the exit LW' for a buried layer in the 1)(I'T'. 'The chemical composition of the buried layer and all covering layer's must be defined.

1. From the main menu select option 1, "D)k FINI; DUT LAYERS." "The layers of the DUT are numbered from (1, the top layer tos. The last layer entered is the active, buried laver for which the LE'I values will be calculated. 'The Model 5005-'TF software calculates the LAT values by transporting each fission-fragment through the layers.

2. 'T'o define the (irst layer press (), and then enter the layer identification, thickness in micrometers, and the mass density in grams per cubic centimeter. If the (nonzero, parameters are correctly entered, the CHEMICAL. COMPOSITION MENU will appear. 'This menu is used to define the chemical composition of the DUI' layer. 'The chemical ele. ments that make up a layer are numbered from o to 9 .

3. 'T'o enter the first chemical element press 0, and then enter the atomic number of the element and the atoms per molecule of the element in the chemical compound that makes up the DI'I' layer. If the D)(J'T layer is not a true compound, but a mixture, you will need to convert the elemental composition of the mixture into an equivalent atoms per molecule for each element as if the mixture were a chemical compend. Figure 45h shows an example elemental composition of the glass laver of a I) l'T'.

4. Press the space bar to exit, and continue the above procedure unt il each layar of the I) I'T' is defined. Figure doa shows an example lon definition.

5. Press the space bar to exit to the main menu. 
DEFINE DU'T LAYERS (defined) new

$\begin{array}{cc}\text { PRESS TO } & \text { LAYER } \\ \text { SELECT } & \text { IDENTIFICATION }\end{array}$

$\begin{array}{cc}\text { DENSITY } & \text { THICKNESS } \\ \mathrm{g} / \mathrm{CC} & \mathrm{Um}\end{array}$

glass

a luminum

epi silicon
0.800

1.400

5.000

0.000

0.000

0.000

0.000

0.000

0.000

0.000

PRESS THE SPACE BAR TO EXIT

a. DU'I' LAYER DEFINITION MENU

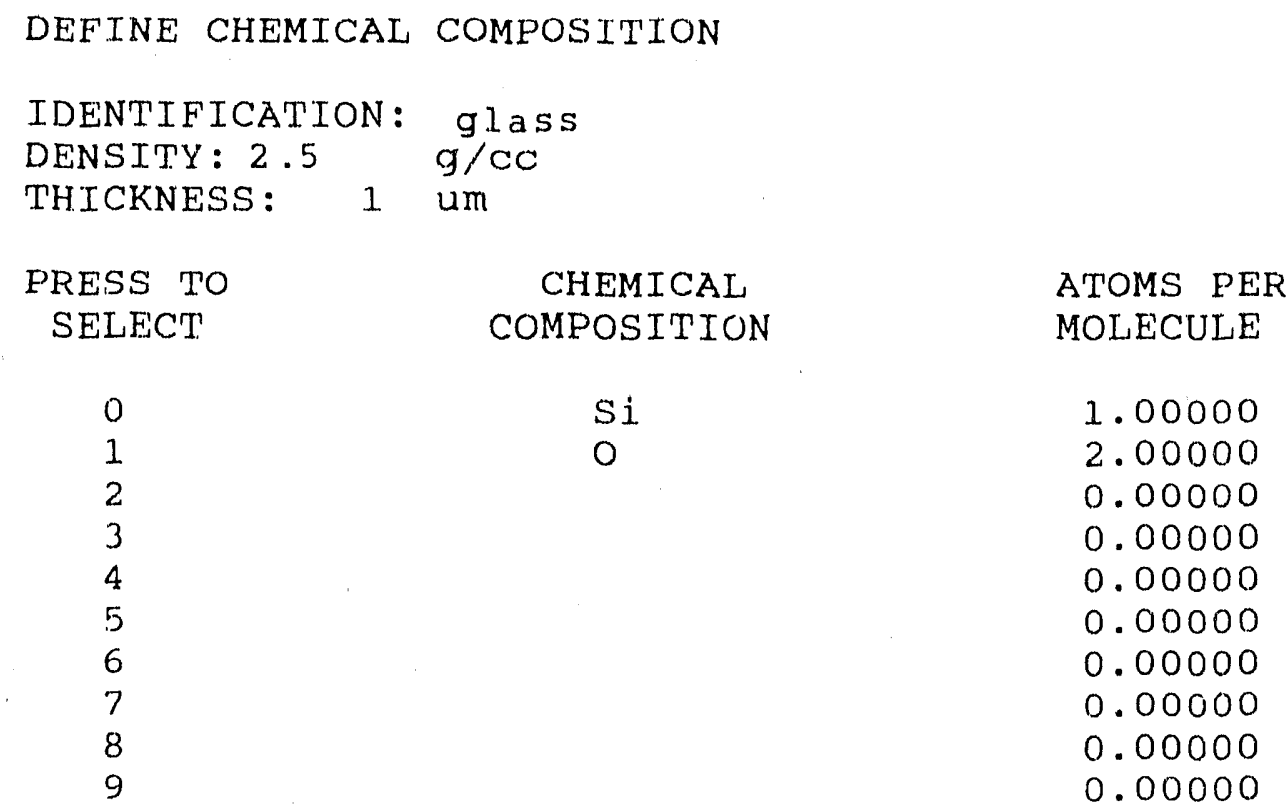

PRESS THE SPACE BAR TO EXIT

b. CHEMICAL COMPOSITION menU

Figure 45. Example of a DU'T definition 
Test Paramoter Specification. Three test parameters must be specified for each single-event test of the DUT: (1) the test identification, (2) the angle of incidence of the (1'-252 fission fragments on the I) U'T' (zero degrees is normal incidence), and (3) the characteristics of the thin foil (if any) used to degrade the fission fragments' energies. The degrader foil is optional, supplied by the user, and is mounted between the last TFD and the DU'T. The degrader is characterized by the mass density, the Pl, and PH channel values cobtained from energy spectra of the fission Iragments from the large test source as they pass through both TFD and the degrader foil) and the chemical composition of the degrader foil. Figure 46 is an example of the test parameters for a single-event test in which the fission fragments are normally incident on the DUT' and no degrader foil is present.
Calculation of the Look-Up Table. When the Model $5005{ }^{2} \mathrm{TH}$ has been calibrated, the DUT has been defined, and the test parameters have been specified, then the main menu should have the status flags set as shown in Figure $47 \mathrm{a}$.

1. Press 7, "SAVE TES'T DA'TA," and save the system calibration, the DU' $\mathrm{U}^{\prime}$ definitions, and the test parameters.

2. Press the space bar to exit. 'The main menu should now have the status llags set as in Figure 47b.

3. Press 3, "CALCULA'TE I,OOK-UP 'TABI.E," to begin the calculation of the LACI values of the fission fragments in the DU'T buried layer for each fission fragment. On completion of the calculation, the main menu should have the status flags set as shown in Figure 47c.

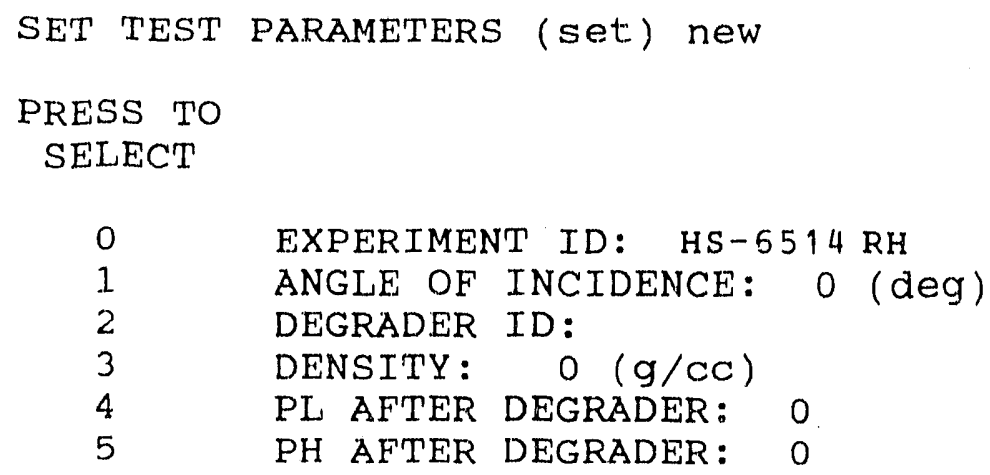

Figure 46. Example SET TEST PARAMETERS 
MODEL 5005-TF SINGLE NVENT TEST SYSTEM

PRESS T'O

SELECT

O CALIBRATE SYSTEM

1 DEFINE DUT LAYERS

SET TEST PARAMETERS

CALCULATE LOOK UP TABLE

BEGIN SINGE EVENT TEST

DISPLAY TEST DATA

PRINT DATA SUMMARY

SAVE TEST DATA

CLEAR TEST DATA

RECALL TEST DATA

(calibrated)

(defined)

(set)

(not ready)

(not ready)

(not ready)

(ready)

(ready)

(ready)

(ready)

PRESS THE SPACE BAR TO EXIT

a. System calibrated, DUT defined and test parameters set

MODEL 5005-TF SINGLE EVENT TEST SYSTEM

PRESS TO

SELECT.

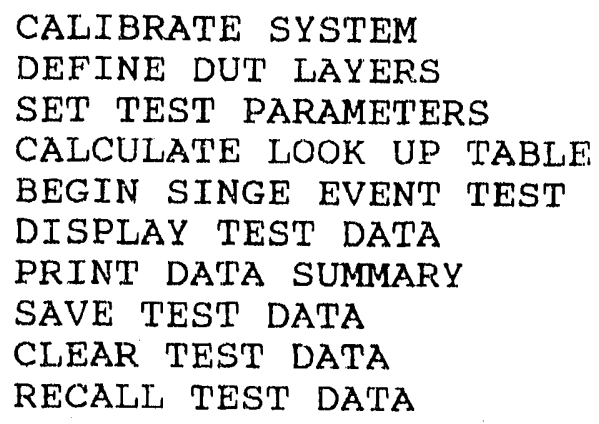

CALIBRATE SYSTEM

DEFINE DUT LAYERS

SET TEST PARAMETERS

CALCULATE LOOK UP TABLE

BEGIN SINGE EVENT TEST

DISPLAY TEST DATA

PRINT DATA SUMMARY

SAVE TEST DATA

CLEAR TEST DATA

RECALL TEST DATA

(calibrated)

(defined)

(set)

(ready)

(not ready)

(not ready)

(ready)

( ready)

( ready)

(ready)

PRESS THE SPACE BAR TO EXIT

b. System ready to calculate look-up table

Figure 47. Main menu status flags (continued) 
MODEL 5005-TF SINGLE EVENT TEST SYSTEM

PRESS TO

SELECT

$\begin{array}{ll}0 & \text { CALIBRATE SYSTEM } \\ 1 & \text { DEFINE DUT LAYERS } \\ 2 & \text { SET TEST PARAMETERS } \\ 3 & \text { CALCULATE LOOK UP TABLE } \\ 4 & \text { BEGIN SINGE EVENT TEST } \\ 5 & \text { DISPLAY TEST DATA } \\ 6 & \text { PRINT DATA SUMMARY } \\ 7 & \text { SAVE TEST DATA } \\ 8 & \text { CLEAR TEST DATA } \\ 9 & \text { RECALL TEST DATA }\end{array}$

(calibrated)

(defined)

(set)

(calculated)

(ready)

(not ready)

(ready)

(ready)

(ready)

( ready)

PRESS THE SPACE BAR TO EXIT

c. System ready for single-event testing of $\mathrm{D}) \mathrm{U}^{\prime} \mathrm{C}^{\prime}$

\author{
MODEL 5005-TF SIINGE EVENT TEST SYSTEM \\ PRESS TO \\ SELECT' \\ O CALIBRATE SYSTEM \\ 1 DEFINE DUT LAYERS \\ 2 SET TEST PARAMETERS \\ 3 CALCULATE, LOOK UP TABLE \\ 4 BEGIN SINGE EVENT TEST \\ 5 DISPLAY TEST DATA \\ 6 PRINT DATA SUMMARY \\ 7 SAVE TEST DATA \\ 8 CLEAR TEST DATA \\ 9 RECALL TEST DATA \\ PRESS THE SPACE BAR TO EXIT \\ d. System ready to display previous test data
}

(calibrated)

(defined)

(set)

(calculated)

(acquired)

(ready)

( ready)

( ready)

(ready)

(ready)

Figure 47. Concluded 


\section{Acquiring Single-Event Data}

1. Initialize the DUT (using the event-detection (ircuit)

2. Press 4, "BECIN SINCiLE EVEN'i 'TES'T" from the main menu. A display of the DUT single-event total cross section similar to Figure 48 should appear.

3. If the display does not appear, reset the ADC unit. The total cross section is calculated and displayed as the single-event data accumulate. The total particle fluence is displayed in the upperer right hand corner of the plot.

4. The single-event test is terminated by pressing the space bar.

Reviewing Test Data. The cross section vs. IET' curve can be displayed for any single-event code. The display is a function of the surface, average-effective, or exit IET using the DU'T definition and test parameters as specitied before the single-event test, or as currently speciilied. This allows any errors made in the DUT' definition and test-parameter specifications to be corrected. To select option 5, "DISPLAY TEST DATA," the main menu status flays must be set as in Figure $47 \mathrm{c}$ or Figure $47 \mathrm{~d}$.
Other Software Functions. The remaining options (6) through 9 ) of the main menu permit obtaining a printed data summary and manipulation of system data files (save, clear, and recall). All data files have a suffix version number from 000 to 999. Thus there can be up to 1000 versions of any data file. The system data files are organized as a pyramid with the calibra. tion data file, SYSCAL, at the pinnacle. For each (past) system calibration, up to 1000 different DUT definition files, DEFDUT, are possible. For each DUT' definition file there can be up to 1000 different test parameter files, SETTST. Each time a look-up table is calculated, the SYSCAL, DEFDUT, and SE'TIS'T version numbers are stored in the TABCAL, file. These version numbers are stored with the TOF and LET voltages and LET values in the DUT' active, buried layer. For each set of single-event data, the TABCAL version number is stored in the RADTST' file with the 'TOF and LET' voltages, the SEC, and the LET values.

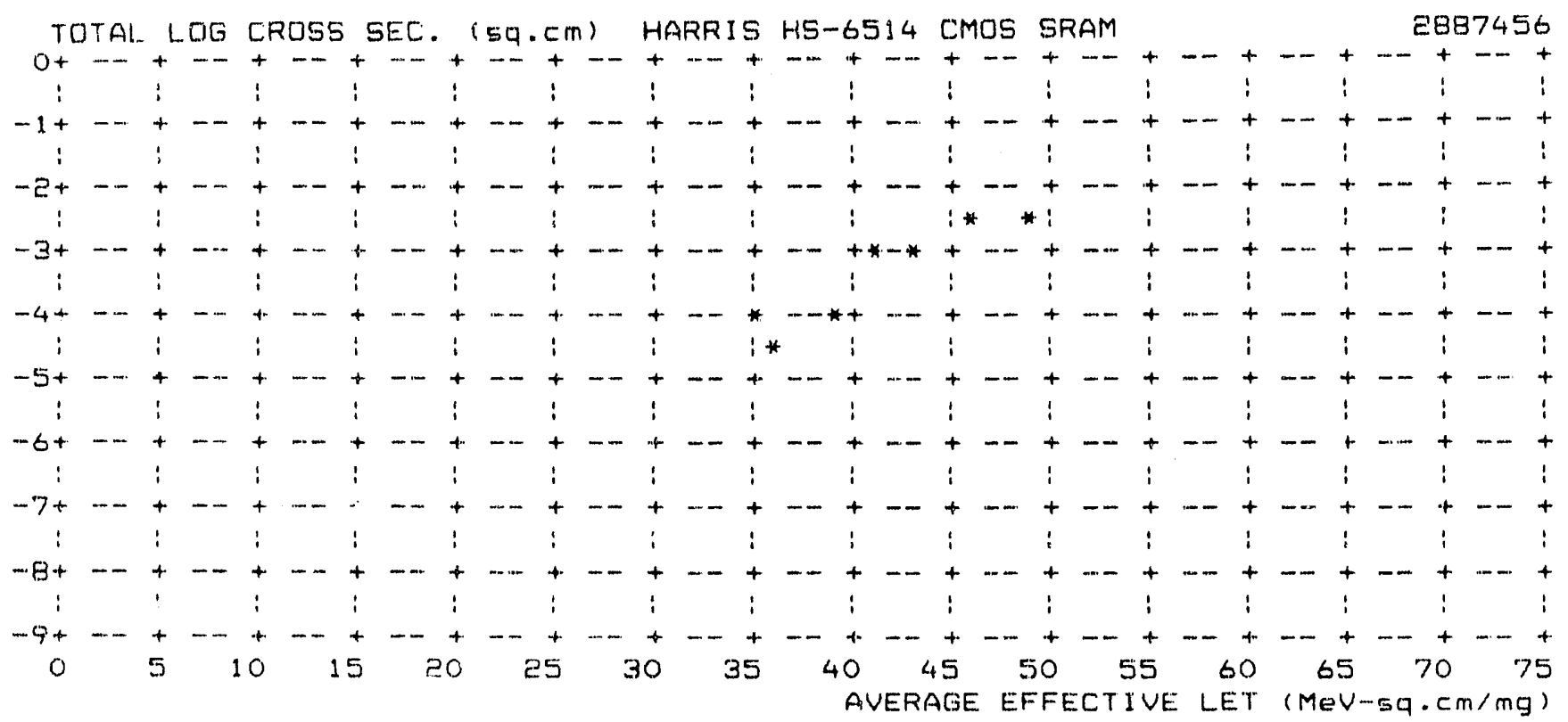

Figure 48. ('ross section vis. LET (urve for the Harris HS-65] 4RH SRAM. Data taken at Lockheed Missiles and Space Company, August 27, 1990). 


\section{Maintenance}

\section{1 PMT and Scintillators}

Maintenance of the PM'T and base units should be minimal and require no at tention unless a problem is experienced (i.e., unacceptable noise level, no output signal, etc.). Refer to Section 6, Trouble shooting.

The thin-film scintillators used are $1 \mu \mathrm{m}$ thick and are extremely fragile.

1. If the foil is damaged, replace it.

2. 'T'o replace the foil, remove the scintillator holder from the PMT'.

3. Loosen the Allen head setscrew and slide the two half-pieces of Lucite plastic out of the aluminum holder (Figure 49).

4. Remove all of the pieces of the old foil and replace them with new ones. ('Try to get the new foil as taut as possible across the hole without breaking or tearing it.)

5. Carefully place the second half of the Lucite plastic light pipe on top of the first and insert it into the aluminum holder.

6. Make sure the $: 3 / 4-i n$. hole in the aluminum holder is aligned with the holes in the lacite.

7. Tighten the setscrew to secure the Lucite in place.

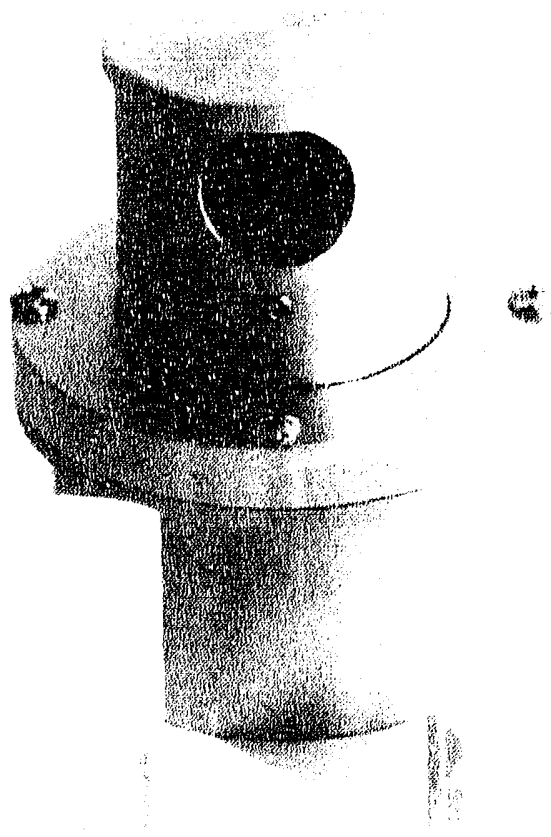

Figure 49. IItra-thin-film scintillation detector light pipe and holder

\subsection{NIM Modules and Bin}

'The NIM modules and bin essentially require no maintenance. Refer to the EG\&G Ortec manuals for additional information.

\subsection{Vacuum System}

The vacuum system requires periodic maintenance of the Alcatel pumping system and the vacuum chamber. The Alcatel pumps should be maintained according to the owner's manuals. The vacuum chamher is constructed using standard vacuum technology. Some sugurestions for maintaining the quality of the vacuum are as follows:

- Vent the system with dry nitrogen gas.

- Keep the inside of the vacuum chamber free of dust and fingerprints.

- Clean and re-grease the exposed O-rings every few months.

- Keep the system closed and pumped down when not in use.

- Clean all DUT fixtures hefore installing them in the vacuum chamber.

\section{Trouble Shooting}

This section helps you solve problems with the Model 5005-TF. 'To use the problem determination procedures below, first find the description of the symptom of the problem. Then read the probable cause and remediy. If the remedy does not correct the problem, then contact sipectrum siciences.

\subsection{Section 2.3/System Setup and Preparation for Use}

symptom: Oil is coming from the oil-mist eliminator.

Probable Cause: The internal cartridge is saturated with oil.

Remedy: Replace the internal cartridge.

\subsection{Section 2.4 /Installation of the Cf-252 Sources}

simptom: source access shutter will not opent.

Probable ('ause: The sistem is nol completelyented.

Remedy: Complete the venting presedure

Sismptom: The modified large test soure will not fit into the soure holder. 
Probable Cause: The source holder is not properly aligned.

Remedy: Realign the source holder.

\subsection{Section 4.2 /System Pumpdown}

Symptom: The system will not achieve a high vacuum.

Probable Cause:

- The vent valve is open.

- An electrical feedthrough is missing or leaking.

- An O-ring is leaking.

- A foreign substance inside the chamber is cutgassing.

- The vacuum controller needs servicing.

- The pumping system is not working properly.

Remedy:

- Check to see if the vent valve is closed.

- Repair or replace any leaking electrical feedthroughs.

- Examine the O-rings for wear and ensure that they are seated properly.

- Remove all items from the chamber and then try to pump down the system.

- Refer to the GP and Alcatel manuals for additional problem determination procedures.

\subsection{Section 4.3 /System Calibration}

Symptom: 'The PMT's arc when the high voltage is applied.

Probable Cause: 'The system is not pumped down to a high vacuum.
Remedy: Review the system pump-down procedures.

Symptom: 'The PM'T's draw large currents when the high voltage is applied.

Probable Cause: The ion gauge has been left on.

Remedy: Turn off the ion gauge.

Syrnptom: Pulse-height spectra cannot be obtained from the particle detectors.

Probable Cause:

- The NIM electronics are not cabled correctly.

- The detector power supplies or NIM bin are not powered.

- A de power supply in the NIM bin has failed.

- A thin-film scintillator is broken.

Remedy:

- Check the system cabling.

- Verify that the power supplies are on.

- Verify that all NIM module switches are set correctly.

- Test the dc voltage values on the side of the NIM bin.

- Replace the broken scintillators.

Symptom: 'The ADC unit does not appear to function during the system calibration or singleevent testing.

Probable (ause: The power-on reset has failed, or the ADC: unit is not cabled properly.

Remedy: Reset the ADC unit using the front-panel switch. Verify that the unit is cabled correctly. 


\section{References}

'D). K. Nichols, "SFel' T'esting," yh International ('on. ference on lon-Beam Analysis, Kingstom, ()ntario, Canada, June $24-330,1969$.

M. L. Muga, 1). . B. Burnsed, W. E. Sleverer and H. F. Taylor, "A New 'Time-of. Flight P'artele Delector," Nuclear Instruments and Metheds sis (1976) 13:5-138.

M. L. Mugat. "A Versatile dF/dx Detector for Heave Mass Nuclear Particles," Nucle'ar Instrumentis and Meth. ads 95 (1971):349-359.

'T. Muga. "Response' of 'Thin-film Sicintillator l)eter. tors to High-Finergy Argon and Krypton Lons," Naclear Instruments and Methrods i2. North.Helland Hub. ('o. (1975) 5.11 .546

M. L. Muga and .I. D. Bridgess, "Sipecific Lominesrence Studies of Alpha Particles In NF. low Scintillator Film." Nuedear Instruments and Methods li3t (1976) bai147.

"R. K. Batra and A. (". Shotter, "Passage of Fission Fragments 'Through 'Thin Plastic Scintillators," Nuciear Instruments and Methods in Mhusias Researeh B.5 (1984) 1.1.19.

'F. I). Bronks, W. A c 'illiers and M. S. Allie, "Response" of Thin NEllo:A Sicintillator Films to Fission Fragments,"

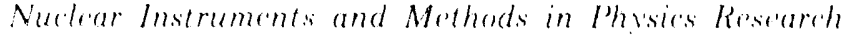
A2.10 (1985) 338.342.

"(". Manduchi, M, T', Russo-Manduchi and (i, F, Stuato. "Response of Illtra-Thin Sointillator Foils to Fis- sion Fragments," Nuelear Instruments and Mothends in

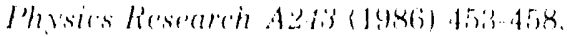

"I. Kammer and Y. Nakaseme, "A New Model of lumi nescence Production in a Very Thin Plastic Scoutillator

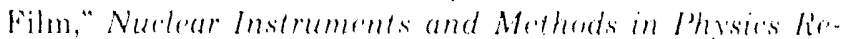

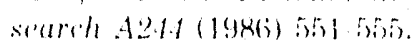

"I. Kammo and Y'. Nakagome, "Response (hatracteris. tics of Thin Film l)etectors to lissiom Fragments," Nuclear

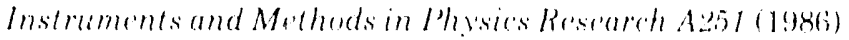
$108-114$.

"1). Mapper, 'T. K. Sanderson, J. H. Stephen, J. Farren, L. Adams, and R. Harberesorenson, "An Fxperimental Study of the Eiffect of Absorbers on the lete of the Fission

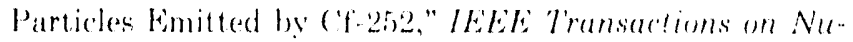

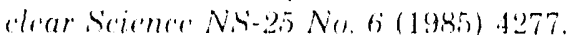

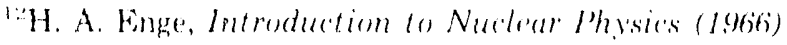
Addison-Wester, dan.

13. Segre, W. A. Benjamin, Nuclei and Particles (1977) 587.

'il. ('. Nurtheliffe and R. F. Shohilling, "Range and Stopping Power Tables for Heave lons," Nuclecar Mata A7 (1970) Academic Press, 236.

1:J. F. Ziegler, Handbenk of Stopping ('ross Soctions. for Energetic lons in All Eirments 6, Pergamon Press (1980) 19.

"ii.J. Weisman, Elrments of Nuclear Reartor lesign Elsevier (1977) 346.

"(i. F. Knoll, Radiation I)etertion and Metasurement, John Wiley d Soms (19793) a) 


\section{APPENDIX A}

\section{Example Standard Operating Procedures}

for Cf-252 Sources 
SOP NUMBER

HP 900229002

Responsible Org. 9351

Safe Operating Procedure

For the

Californium - 252 Fission Fragment Source Division 9351 Lab Area

This document contains safe operating procedures for the Californium - 252 Fission Fragment Source in the Division 9351 Laboratory Area. Improper use or handling of this source could result in serious personal injury.

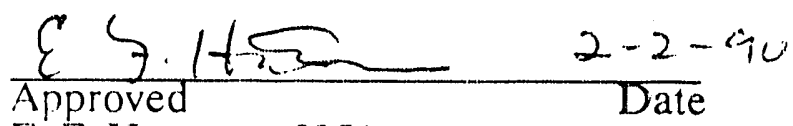

E. F. Hartman, 9351

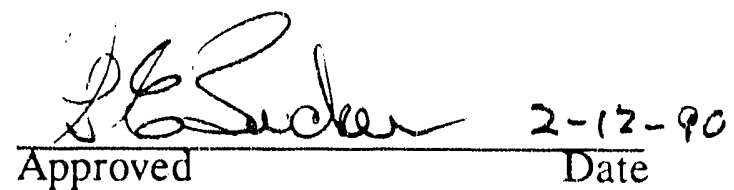

G. E. Tucker, 3212

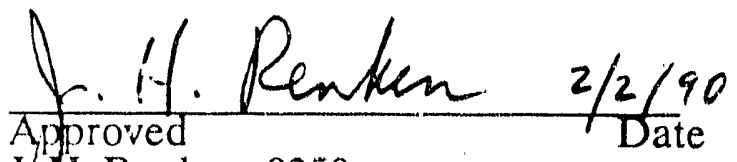

JUH. Renken, 9350

5) 


\section{Safe Operating Procedures \\ for the \\ Californium - 252 Fission Fragment Source Division 9351 Lab Area}

\section{General}

1. All persons designated or authorized to operate this equipment shall have a full understanding of current operating procedures.

2. A copy of these procedures will be posted in a conspicuous place in the work area.

3. Californium - 252 is an inherently dangerous material. It is a source of gamma rays, alpha particles, neutrons, and fission fragments. Ingestion of small quantities of Californium can result in long term health hazards. Therefore, these procedures shall be read and understood before using the source.

\section{Specific}

1. Rubber gloves are to be used if the source is handled and when the source is removed or installed in the borated shield or the storage cabinet. When removing the gloves, care should be taken to assure that any contamination will remain inside the removed glove. After each removal of the rubber gloves, your hands must be washed with soap and water. Gloves should be stored in a plastic bag and checked by health physics for contamination before disposal.

2. When the source is not under vacuum and a test is finished, the source must be removed and stored in the locked, borated neutron shield or in a designated plastic box in the locked storage cabinet.

3. Care should be taken to never contact the sealed surface of the Cf-252 source in any manner with any device or tool.

4. The borated shield must be locked when unattended.

5. When the source is in the vacuum chamber or in the borated shield, a highly visible sign will be placed on the vacuum chamber identifying the radiation hazard.

6. Health Physics will provide a contamination wipe twice a year of the vacuum chamber, source and plastic storage container.

7. If contamination is observed, the source can no longer be used until cleared by Health Physics.

8. If it is found necessary to deviate from these procedures, contact E. F. Hartman, Division Supervisor, 9351, and the Health Physics Division 3212. 


\section{LIST OF AUTHORIZED USERS}

NAME

J. S. Browning
DATE

$2-2-90$
ORG. SIGNATURE

9351

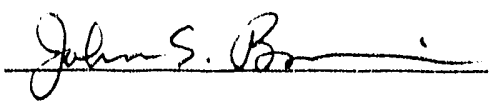

By my signature above, I indicate and agree that I have read the Safe Operating Procedure for the Californium - 252 Fission Fragment Source, and that I will abide by the practices and rules contained within this procedure. 


\section{APPENDIX B}

\section{Dual-Channel Pulse-Height ADC Circuit Schematic}



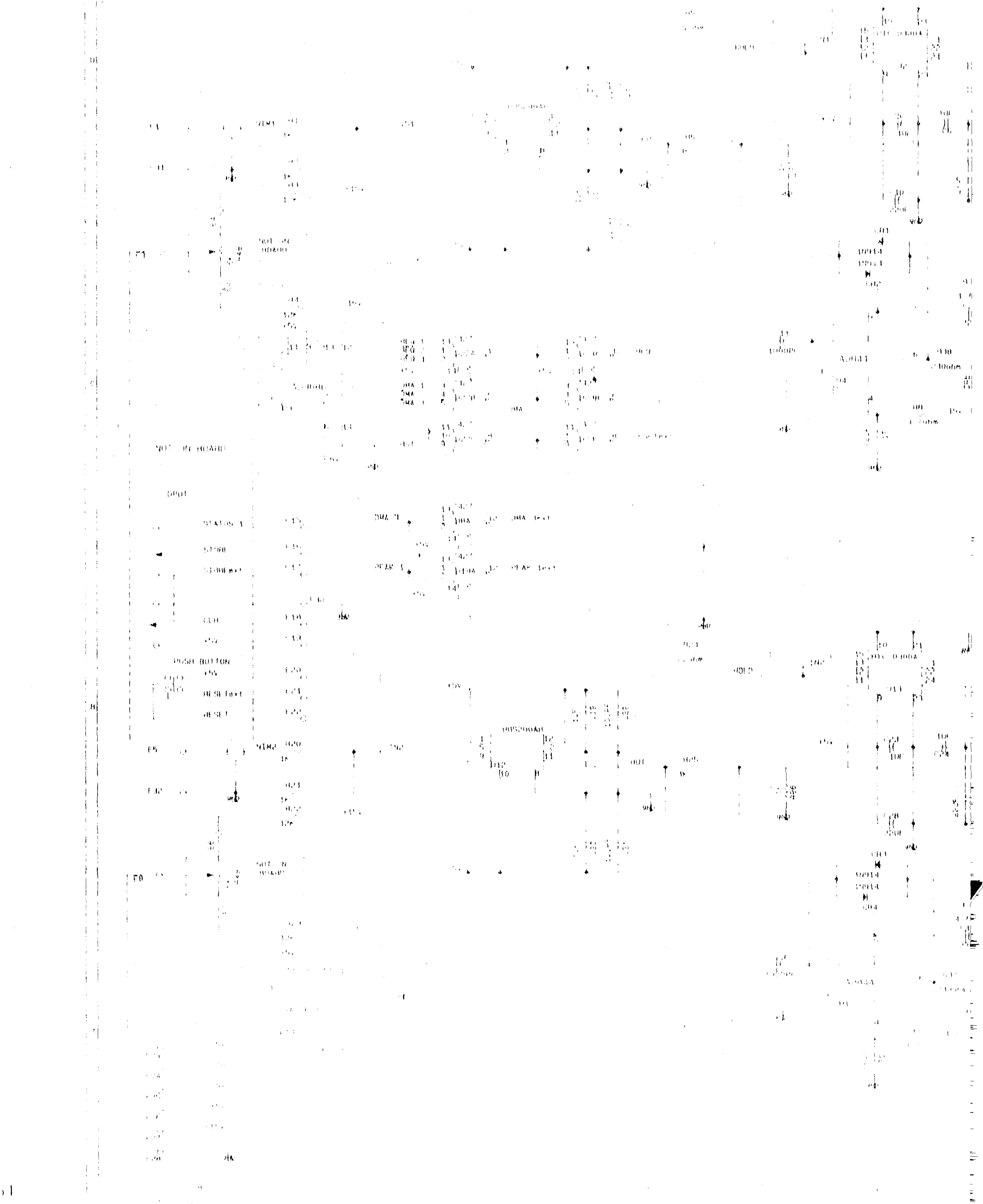


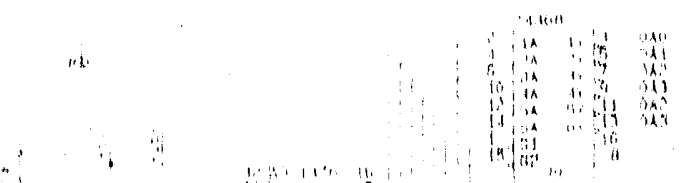

......
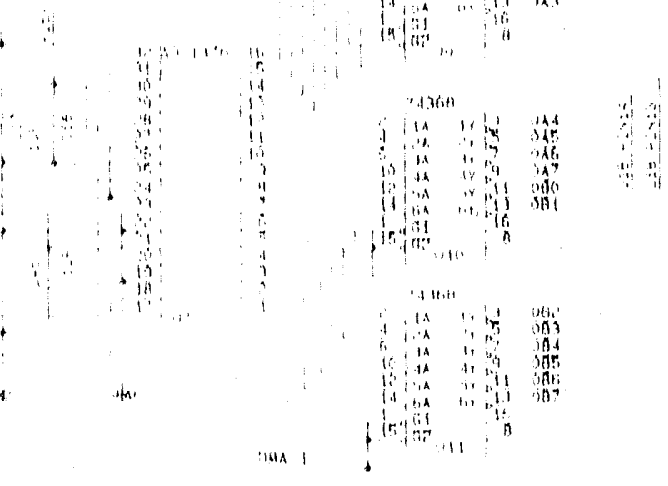

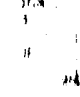

ind

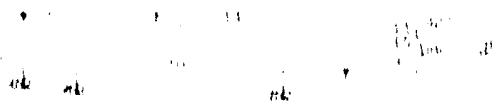
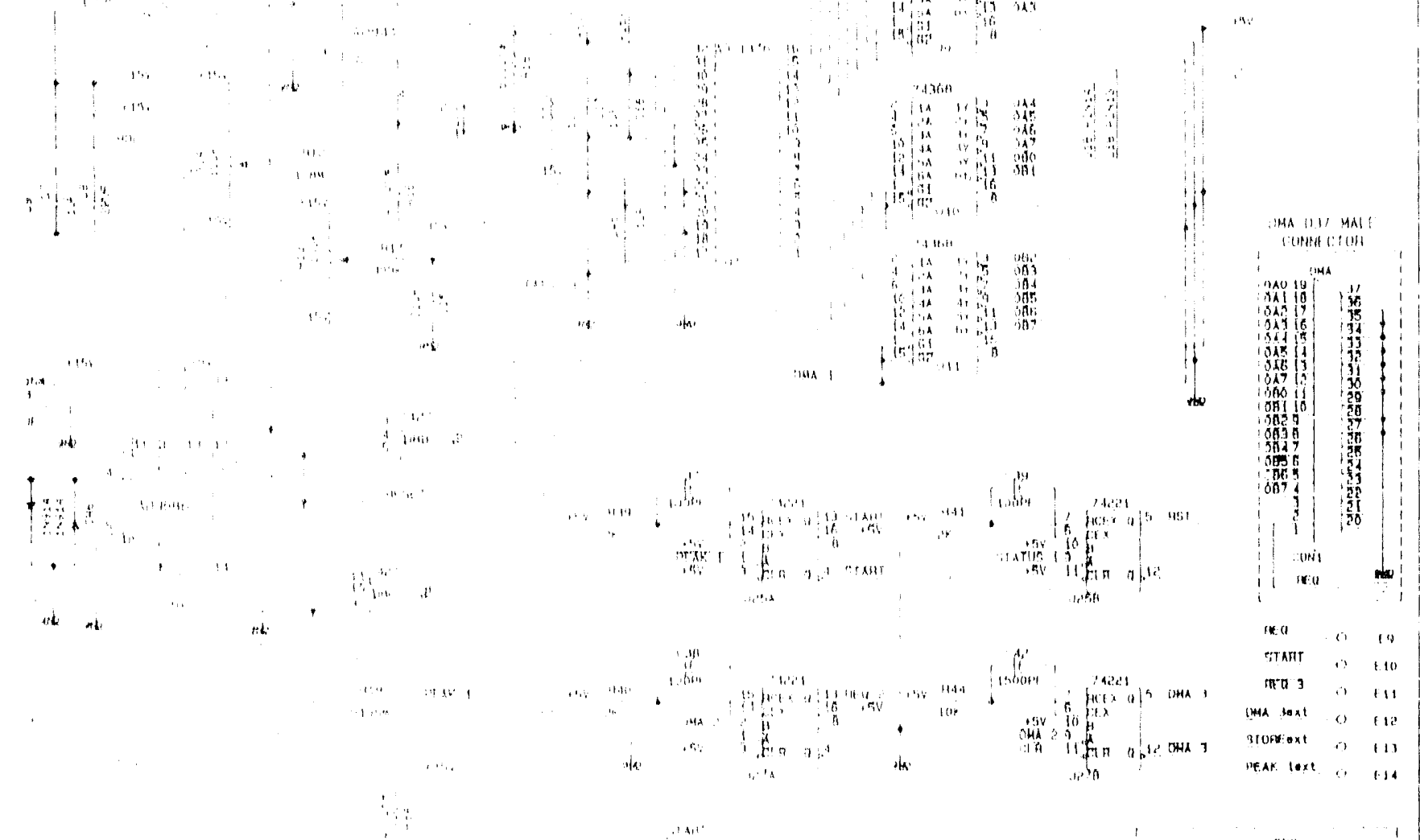

,

....

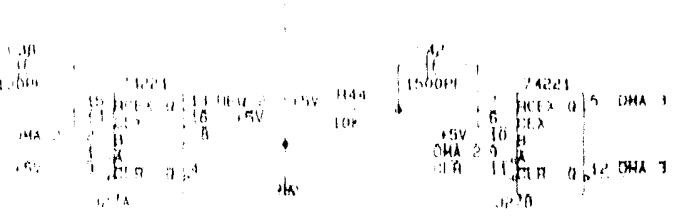

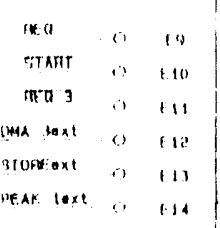
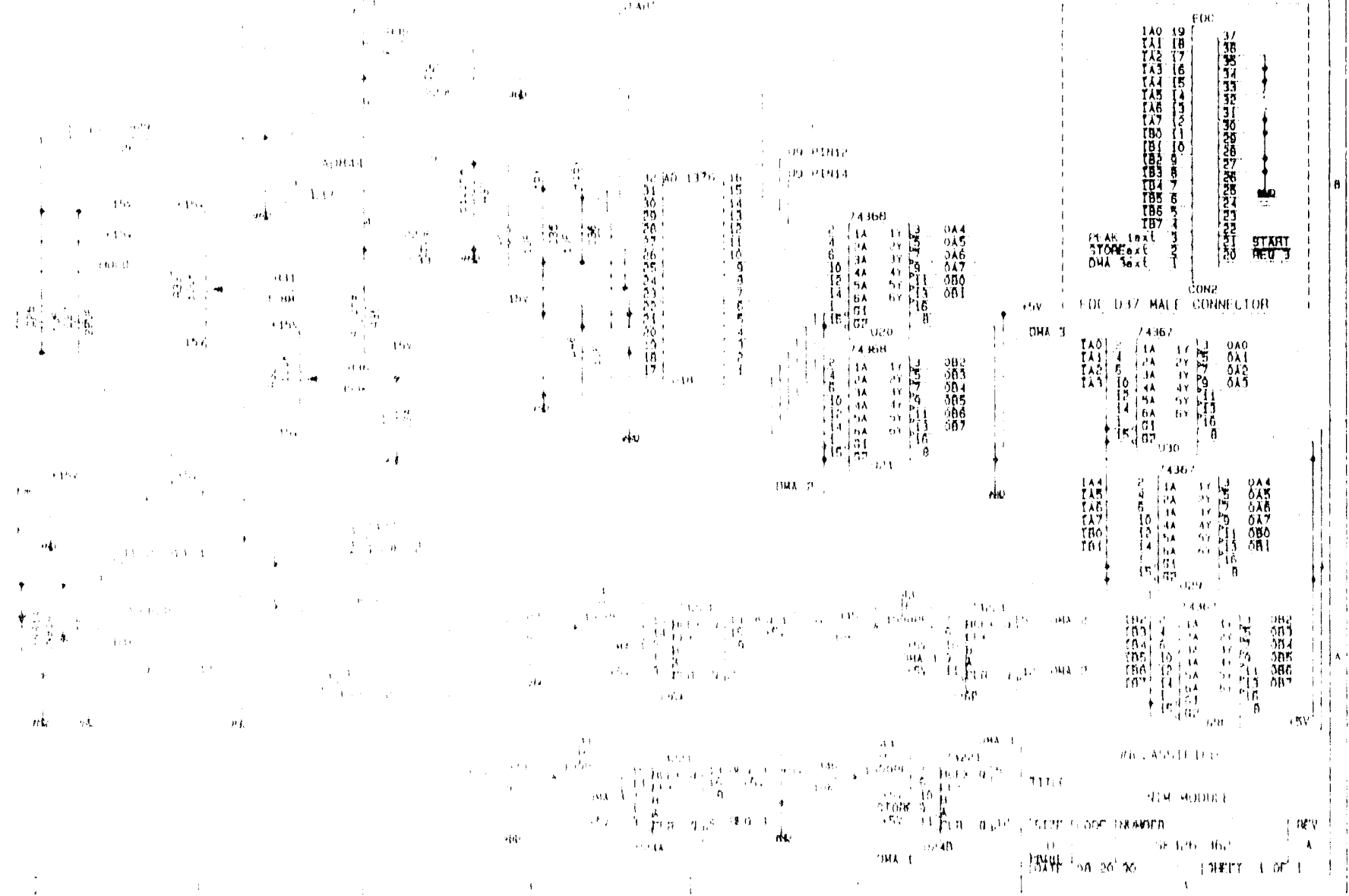
APPENDIX C

Software Description 
a. Software Modules.

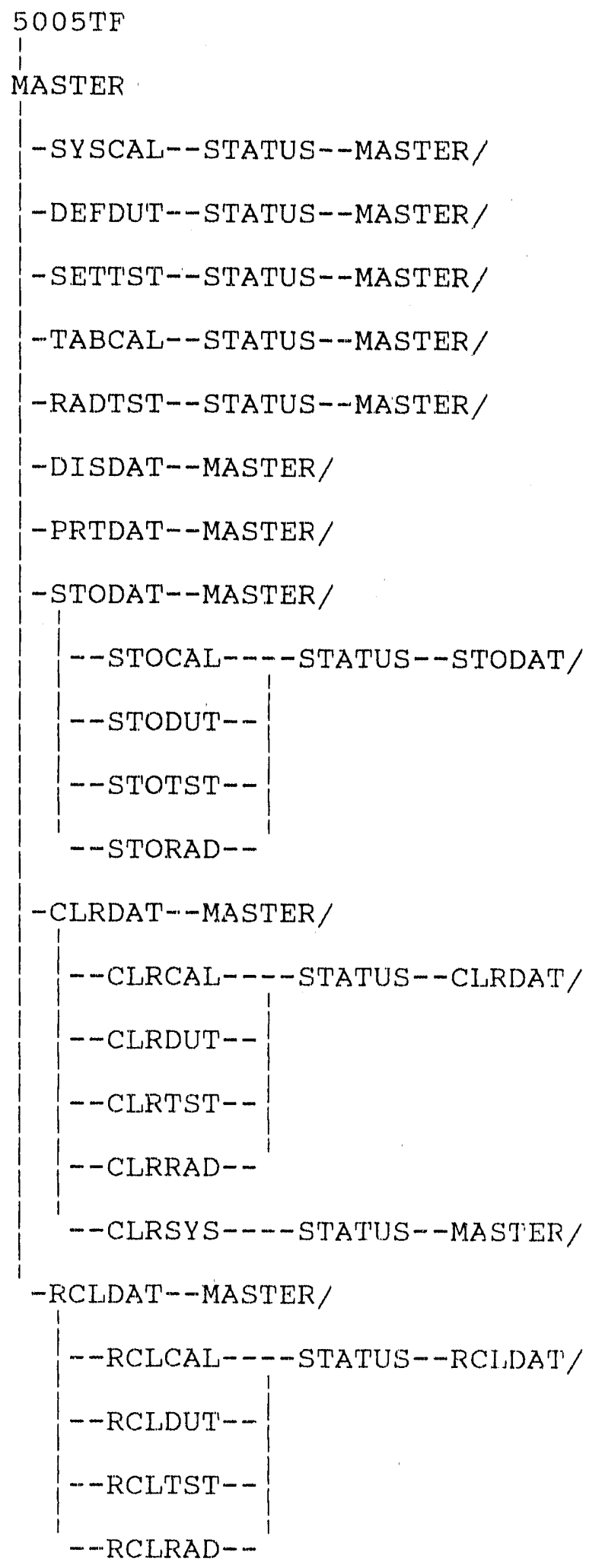


b. Module 5005TF.

The 5005TF executable module loads the ADC gain and zero offset corrections from data file 5005TF.DAT. The 5005TF module calls the MASTER module. The 5005TF.DAT record format is:

"5005TF. DAT"

" $<$ ADC unit serial number $>$ "

$<$ TOF gain $>,<$ TOF zero offset $>$

$<$ LET gain>, <LET zero offset>

EOF

\section{Module MASTER.}

The MASTER executable module implements the main menu. Based on the selected option (0-9) it calls modules: 0) SYSCAL, 1) DEFDUT, 2) SETTST, 3) TABCAL, 4) RADTST, 5) DISDAT, 6) PRTDAT, 7 ) STODAT, 8) CLRDAT, and 9) RCLDAT. There is also an option to exit to DOS. The system status flags are displayed and control the selection of options. The system status information is stored in the STATUS.DAT file. The record format of the STATUS.DAT file is:

"STATUS.DAT"

" 0 CALIBRATE SYSTEM","<status flag: '(uncalibrated)' or '(calibrated)'>" "<active SYSCAL version or 'new'>", <highest SYSCAI version number 000 - 999>

" 1 DEFINE DUT MATERIAL LAYERS","<status flag: '(undefined)' or '(defined)'>", "<active DEFDUT version or 'new'>", <highest DEFDUT version number 000 - 999>

" 2 SET TEST FARAMETERS","<status flag: '(clear)' or '(set)'>", "<active SETTST version or 'new">", <highest SETTST version number 000 - 999>

" 3 CALCULATE LOOK UP TABLE","<status flag: '(not ready)', '(ready)' or '(calculated)'>"," <active TABCAL version or 'new'>", $<$ highest TABCAL version number 000 - 999>

$" 4$ BEGIN RADIATION EXPOSURE", "<status rlag: '(not ready)', '(ready)', or '(acquired)'>","<active RADTST version or 'new'>", <highest RADTST version number 000 - 999>

" 5 DISPLAY TEST DATA","<status flag: '(not ready)' or '(ready)'>", "new", 0

" 6 PRINT DATA SUMMARY","<status flag: '(not ready)' or '(ready)'>", "new", D 
$" 7$ SAVE TEST DATA","<status flag: '(not ready)' or

'(reacy)'>", "new", 0

" 8 CLEAR TEST DATA","<status flag: '(not ready)' or

'(ready)'>","new", 0

" 9 RECALL TEST DATA","<status flag: '(not ready)' or

'(ready)'>","new", 0

EOF

d. Module STATUS.

The STATUS executable module reads the STATUS.TMP file, updates the records of the STATUS.DAT file, and calls the executable module specified in the STATUS.TMP file. The record format of the STATUS.TMP file is:

"STATUS TMP"

"<module to be called after updating the STATUS.DAT file>"

<record number of the STATUS.DAT file to be updated>

"<status flag>"

"<active fille version or 'new'>"

$<$ highest file version number 000-999>

EOF

e. Module SYSCAL.

The SYSCAL executable module implements a menu for obtaining the system calibration data and updates the SYSCAL.DAT file, and creates the STATUS.TMP file. Either the MASTER or ADCCAL modules are then called by the STATUS module. If the ADCCAL module is to be called (to calibrate the ADC unit) then the SYSCAL module omits the last two records from the SYSCAL.DAT file. The record format of the SYSCAL.DAT file is:

"SYSCAL. DAT"

<calibration source light fission fragment peak channel number> <calibration source heavy fission fragment peak channel number> <test source light fission fragment peak channel number> <test source heavy fission fragment peak channel number> $<$ ight fission fragment peak channel number after first TFD> 
heavy fission fragment peak channel number after first TFD>

<light fission fragment peak channel number after second TFD >

<heavy fission fragment peak channel number after second TFD>

$<$ collimator area in $\mathrm{sq} . \mathrm{cm}>$

<separtion between TFD in $\mathrm{cm}>$

<TOF light peak voltage>, <LET light peak voltage>

$<$ TOF heavy peak voltage $>$, LET heavy peak voltage>

EOF

f. Module aDCCAL.

The ADCCAL executable module is used to calibrate the TOF and LET voltage pulse heights. This is accomplished by

identification of the light and heavy mass fission fragment

peaks. The user has the option of reviewing previous calibration

data from the ADC unit, or acquiring new calibration data. If

the user opts to review the previous data, stored in file

ADCCAL.DAT, then a two dimensional plot of the TOF and LET

voltage pulse heights is constructed with the number of events represented by the single digit exponent of the number of counts recorded in the ADCCAL.DAT file for each voltage pair. If the user opts to acquire new calibration data, then the ADCCAL module initilizes the Metrabyte PDMA-16 board, sets up a DMA transfer for two 16-bit words, and waits for the transfer to occur. (The $A D C$ urit must have the upper right-hand switch set to the 'CAL' position.) When a particle event occurs the ADCCAL module writes the voltage pair to the ADCCAL.DAT file and increments the number of counts for the voltage pair. When 100 events have occured, the ADCCAI module updates the two dimensional plot display for the user. The user terminates the data acquisition mode by pressing the space bar. Then the ADCCAI module locates the peak values of the voltage pair counts for the heavy and light fission fragments, appends the values to the SYSCAL.DAT file, and calls the STATUS module. The record format of the ADCCAL.DAT file is:

"ADCCAL. DAT"

$<$ TOF voltage $>$, <LET voltage $>$ 


\section{g. Module DEFDUT.}

The DEFDUT executable module implements the "DEFINE DUT LAYERS" and the "DEFINE CHEMICAL COMPOSITION" menus for user definition of the materials that comprise the DUT. The user may specify the mass density and thickness for up to 10 layers of the DUT, and define a ch mical compound for each layer consisting of up to 10 different chemical elements. The last layer to be defined is assumed to be the active layer in which all charge collection will occur for the single event mechanism. The DuT definition is stored in the DEFDUT.DAT file, which has the following record format:

"DEFDUT. DAT"

"<layer identification, default " ">", <mass density>, <thickness> <atomic number, default 93>, <atoms per molecule>

(The above format is repeated 10 times.)

EOF

\section{h. Module SETTST.}

The SETTST executable module implements the "SET TEST PARAMETERS" menu, stores the test parameters in the SETTST.DAT file, and calls the STATUS module. The SETTST.DAT file has the following record format:

"SETTST.DAT"

"<test id, default " ">"

<angle of incidence>

"<degrader id, default " ">"

$<$ PI for degrader $>$

$<\mathrm{PH}$ for degrader $>$

<atomic number, default 93>, <atoms per molecule>

. $\quad$. $\quad(10$ records $)$

EOF

i. Module TABCAL. 
The TABCAL executable module calculates the look up table which associates the TOF and LET voltage pairs with the surface, average effective, and exit LET values for each fission fragment in the active DUT layer. The first step of the calculation is to determine the thicknesses of the gold cover layer of the test source, the first TFD, the second TFD, and the degrader foil (if present). This is accomplished by matching the measured energy losses of the average heavy and light fragments (stored in the SYSCAL data file) with a thickness that gives the correct energy loss. The technique used is a binary search to select thicknesses that give the energy loss to less than $1 \%$ of the measured values. The energy loss is calculated from integration of the stopping power using a twelve point Gaussian quadrature. The thicknesses for the heavy and light fission fragments are then averaged to obtain an estimated thickness for the gold layer in the test source, the first and second TFDs, and the degrader foil.

The next step is to generate the ensemble of all possible fission fragments from $A=77$ to $A=171$ by simulated fissions that yield one to seven neutrons for each mass number. The atomic number of each fission fragment is estimated from the most probable charge. Each fission fragment is propagated through the gold cover layer, the first and second TFDs, the degrader foil, and the layers of the DUT. The surface, average effective, and exit values of LET are calculated for each fission fragment in the last layer of the DUT (assumed to be the active layer for single events). The TOF and LET voltage pulse heights are estimated from a linear relationship between the pulse heights for the peak values of the heavy and light mass fragments, and the velocities of the average heavy and light fragments between the TFDs (TOF pulse height) and the average velocity of the fragments in the second TFD (LET pulse height). The pulse heights and LET values are stored in the TABCAL data file, which has the record format:

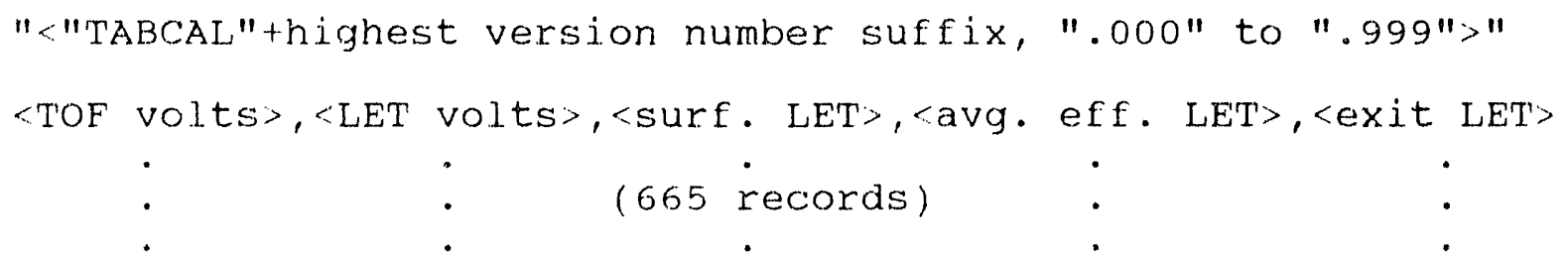

EOF

j. Module RADTST.

The RADTST executable module is used to acquire single event data for a DUT. The total cross section vs. surface, average effective. or exit LET develops as the single event data accumulates. The RADTST module first loads the look up table calculated by the TABCAL module. Then the PDMA-16 board is initialized, a DMA transfer of three 16-bit words is set up, and 
the module waits for a transfer to occur. (The ADC unit must have the upper right-hand switch set to the 'DUT' position.) When a particle event occurs the RADTST module uses the look up table to associate LET values with the TOF and LET pulse heights, writes the voltage pair, LET values, and single event code (SEC) to the RADTST data file, and calculates the single event cross section for the LET values and the SEC. (The SEC \&HFFFF is used to code the event that no single event was detected in the DUT.) The user terminates the data acquisition by pressing the space bar. The record format of the RADTS'P data file is:

"<"RADTST"+highest version number suffix, ".001" to ".999">" $<$ TOF volts>, <LET volts>,"<SEC>", <surf. LET>, <avg. eff. LET>, $<$ exit LET>

EOF

\section{k. Module DISDAT.}

The DISDAT executable module allows the user to review single event data stored in the RADTST data files. The module will display the cross section Vs. surface LET, average effective LET, or exit LET for any single event code. Prior to executing the DISDAT module the user may change any parameters in the SYSCAL, DEFDUT, or SETTST data files. These changes will result in the creation of a temporary, higher version of the RADTST data file. Upon exiting the DISDAT module the user is given the option of saving the new version of the RADTST data file.

\section{Module PRTDAT.}

The PRTDAT executable module is used to print a report of the current system data: SYSCAL, DEFDUT, SETTST, and a table of the fluence, total number of single events, and the single event cross section for any SEC as a function of the surface, average effective, or exit LET in $1 \mathrm{MeV}-\mathrm{sq} . \mathrm{cm} / \mathrm{mg}$ increments.

m. Module STODAT.

The STODAT executable module implements the "SAVE TEST DATA" menu, and calls either the STOCAL, STODEF or STOTST modules based on the user's selections.

n. Module STOCAL.

The STOCAL executable module copies the current SYSCAL.DAT and ADCCAL.DAT files into the SYSCAL and ADCCAL data files with 
the highest version numbers.

○. Module STODEF.

The STODEF executable module copies the current DEFDUT.DAT file into the DEFDUT data file with the highest version number.

p. Module STOTST.

The STOTST executable module copies the current SETTST.DAT file into the SETTST data file with the highest version number.

q. Module CLRDAT.

The CLRDAT executable module implements the "CLEAR TEST DATA" menu and call the CLRCAL, CLRDUT, CLRTST, and CLRSYS modules based on the user's selections.

r. Module CLRCAL.

The CLRCAL executable module replaces all records of the SYSCAL.DAT data file with default values.

s. Module CLRDUT.

The CLRDEF executable module replaces all records of the DEFDUT.DAT data file with default values.

t. Module CLRTST.

The CLRTST executable module replaces all records of the SETTST.DAT data file with default values.

u. Module CLRSYS.

The CLRSYS executable module copies the records of the STATUS.CLR file into the STATUS.DAT file.

\section{v. Module RCLDAT.}

The RCLDAT executable module implements the "RECALL TEST DATA" menu and calls the RCLCAL, RCLTST, RCLDUT and RCLRAD modules based on the user's selections.

w. Module RCLCAL. 
The RCLCAL executable module copies the records of the user selected version of the SYSCAL data file into the SYSCAL.DAT file.

x. Module RCLDUT.

The RCLDUT executable module copies the records of the user selected version of the DEFDUT data file into the DEFDUT.DAT file.

y. Module RCLTST.

The RCLTST executable module copies the records of the user selected version of the SETTST data file into the SETTST.DAT file.

z. Module RCLRAD.

The RCLRAD executable module updates the STATUS.DAT file to make the user selected version of the RADTST file available for the DISDAT and PRTDAT modules. 

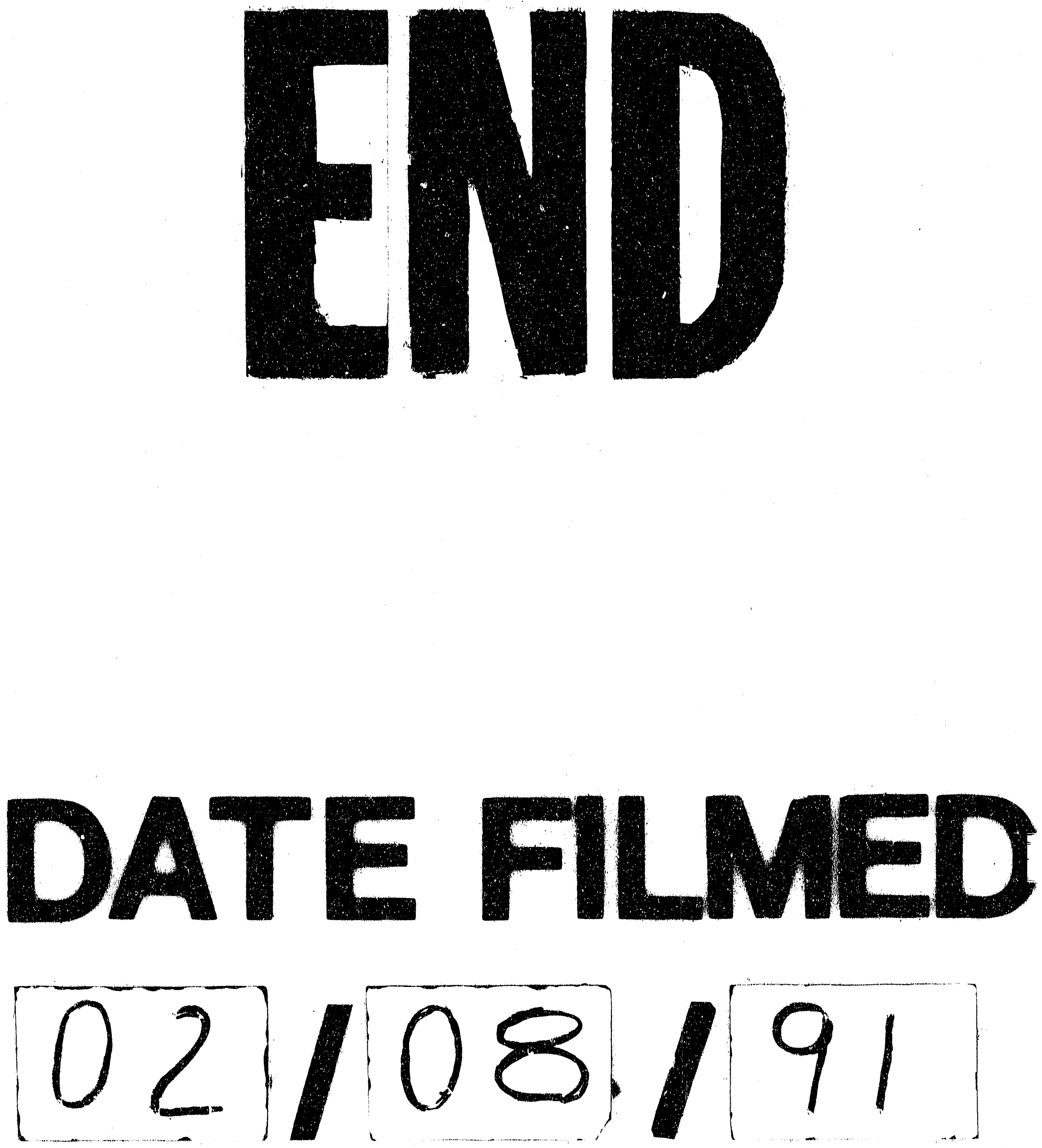
- 